OPEN ACCESS

Edited by:

Benjamin Bahr,

University of Erlangen Nuremberg,

Germany

Reviewed by: Ashkbiz Danehkar,

University of Michigan, United States

Giacomo Cacciapaglia,

UMR5822 Institut de Physique

Nucleaire de Lyon (IPNL), France

*Correspondence:

Manuel Reicher

m.reichert@sussex.ac.uk

Specialty section:

This article was submitted to

High-Energy and

Astroparticle Physics,

a section of the journal

Frontiers in Physics

Received: 14 April 2020

Accepted: 19 October 2020

Published: 24 February 2021

Citation:

Pawlowski JM and Reichert M (2021)

Quantum Gravity: A Fluctuating

Point of View.

Front. Phys. 8:551848.

doi: 10.3389/fphy.2020.551848

\section{Quantum Gravity: A Fluctuating Point of View}

\author{
Jan M. Pawlowski ${ }^{1,2}$ and Manuel Reichert ${ }^{3,4 *}$

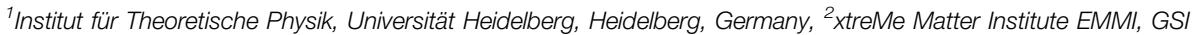 \\ Helmholtzzentrum für Schwerionenforschung mbH, Darmstadt, Germany, ${ }^{3} \mathrm{CP} 3$-Origins, University of Southern Denmark, \\ Odense, Denmark, ${ }^{4}$ Department of Physics and Astronomy, University of Sussex, Brighton, United Kingdom
}

In this contribution, we discuss the asymptotic safety scenario for quantum gravity with a functional renormalization group approach that disentangles dynamical metric fluctuations from the background metric. We review the state of the art in pure gravity and general gravity-matter systems. This includes the discussion of results on the existence and properties of the asymptotically safe ultraviolet fixed point, full ultraviolet-infrared trajectories with classical gravity in the infrared, and the curvature dependence of couplings also in gravity-matter systems. The results in gravity-matter systems concern the ultraviolet stability of the fixed point and the dominance of gravity fluctuations in minimally coupled gravity-matter systems. Furthermore, we discuss important physics properties such as locality of the theory, diffeomorphism invariance, background independence, unitarity, and access to observables, as well as open challenges.

Keywords: quantum gravity, asymptotic safety, background independence, diffeomorphism invariance, ward identities

\section{INTRODUCTION}

One of the major challenges in theoretical physics is the unification of the standard model of particle physics (SM) with quantum gravity. Based on the classical Einstein-Hilbert action, gravity is perturbatively nonrenormalizable and hence cannot be expanded about a vanishing gravitational coupling, the Newton coupling. A very promising way out has been proposed by Weinberg [1], the asymptotic safety scenario. It draws from the theory of critical phenomena developed for investigating the phase structure of condensed matter and statistical systems. In the language of critical phenomena, the standard perturbation theory about a vanishing Newton coupling is an expansion about the free, Gaußian fixed point of the theory and fails since this fixed point is ultraviolet (UV)-repulsive in the relevant couplings. In turn, the asymptotic safety scenario builds upon the conjecture that quantum gravity also exhibits a nontrivial UV fixed point, the Reuter fixed point. This asymptotically safe fixed point should exhibit a finite-dimensional critical hypersurface, which renders the theory finite and predictive even beyond the Planck scale.

The method of choice for respective investigations is the renormalization group. Most investigations of asymptotically safe gravity have been performed with the functional renormalization group (fRG) in its form for the effective action [2]. The fRG approach to quantum gravity has been initiated by the seminal work [3], where the UV fixed point has been studied in the Einstein-Hilbert truncation. In this approximation, one retains only two couplings, the Newton coupling $G_{\mathrm{N}}$ and the cosmological constant $\Lambda$. Already this basic truncation exhibits a UV fixed point in four dimensions $[3,4]$. This exciting finding has triggered a plethora of works for 
asymptotically safe gravity with and without matter. (We refer the reader to the textbooks $[5,6]$ and reviews [7-15]. For very recent accounts of the challenges for asymptotically safe gravity, see 16 , 17. For generic reviews on the fRG, we refer to 18-27.)

The fRG approach to gravity centers around the quantum effective action of the theory $\Gamma\left[\bar{g}_{\mu \nu}, h_{\mu \nu}\right]$, the quantum analog of the classical action. Here, $\bar{g}_{\mu \nu}$ is a generic metric background and the graviton field $h_{\mu \nu}$ accounts for quantum fluctuations about this background. The computation of the effective action $\Gamma\left[\bar{g}_{\mu \nu}, h_{\mu \nu}\right]$ is tantamount to that of the path integral: the $n$-point correlation functions of the dynamical fluctuation field $h$ are given by $n$ derivatives of the effective action with respect to the correlation field, evaluated on the equations of motion, $h=0$ and $\bar{g}=\bar{g}_{\text {EoM }}$, that is, on-shell. These correlation functions are nothing but the moments of the path integral and carry the dynamics of the quantum theory.

This seemingly introduces a background dependence of the approach. However, the approach has inherent on-shell background independence, also related to physical diffeomorphism invariance. Indeed, the background effective action $\Gamma\left[g_{\mu \nu}\right]=\Gamma\left[g_{\mu \nu}, 0\right]$ is diffeomorphism-invariant. The latter properties are the backbones of any quantum gravity approach, and their realization even within approximations is chiefly important.

The present review outlines the properties and results of the fRG approach to asymptotically safe quantum gravity in terms of background and fluctuation correlation functions of gravitons, shortly baptized the fluctuation approach to gravity. This approach is based on the observation that the dynamics of quantum gravity is encoded in the correlation functions of the fluctuation field $h$. Reliable computations of observables can only be done from these correlation functions. This situation calls for a systematic improvement of the standard background-field approximation. In this approximation, the correlation functions of the background metric and the fluctuation field are identified. We refrain from going into more details here, the underlying assumptions and challenges are discussed in Sections 5 and 6.

The fluctuation approach resolves these differences, and by now, it has matured enough to host a large number of results: this includes investigations of the Reuter fixed point in pure gravity in a rather elaborate truncation within a vertex expansion with momentum-dependent two-, three-, and four-point functions; the computation of the background-effective action for backgrounds with constant curvature; investigations of the stability of general gravity-matter systems; investigation of convergence properties of the expansion (apparent convergence); and a potential close perturbativeness of the asymptotically safe $\mathrm{UV}$ regime (effective universality). (We refer the reader to Section $\mathbf{8}$ for an explanation of the terminology and respective results.)

In Section 2, we discuss the general quantum field theory setting of quantum gravity, which we use for the fluctuation approach. This includes a discussion of the necessary gauge fixing and background independence of the approach. In Section 3, we discuss general parametrizations of the full metric in terms of a metric background and a fluctuation field. The preparation in
Sections 2 and 3 allows us to introduce the fRG approach to quantum gravity in Section 4 as well as discussing the standard approximation used in the field, the background field approximation, in Section 5. The symmetry identities that relate the dynamical correlation functions of the fluctuation field and those of the background metric are discussed in Section 6. These symmetry identities imply the necessity to go beyond the background field approximation, and thus, we detail the fluctuation approach in Section 7. With the preparation of the sections before, we discuss the results of the fluctuation approach in Section 8 and close with a short conclusion and outlook in Section 9.

\section{QUANTUM FIELD THEORY APPROACH TO QUANTUM GRAVITY}

The present contribution discusses the advances and open problems of a quantum field theory approach to quantum gravity that is based on the computation of metric correlation functions or, more generally, correlation functions of operators in quantum gravity. Formally, such an approach is based on the existence of a path integral for quantum gravity, for example, defined by the integration over the space of all metrics $\left\{g_{\mu \nu}\right\}$ with a specific classical action for gravity $S_{\text {grav }}$, a standard choice being the Einstein-Hilbert action,

$$
S_{E H}\left[g_{\mu \nu}\right]=\frac{1}{16 \pi G} \int d^{4} x \sqrt{g}(2 \Lambda-R),
$$

with the abbreviation $g=\operatorname{det} g_{\mu \nu}$. In $\mathbf{1}$, we have introduced the Newton coupling $G$ and the cosmological constant $\Lambda$. $R$ stands for the Ricci scalar. In most works in the fRG approach, the theory is considered in its Euclidean version, which is indicated here by the missing minus sign in the square root of the determinant. The expectation value of a diffeomorphism invariant operator $\mathcal{O}\left[g_{\mu \nu}\right]$ is formally given by

$$
\left\langle\mathcal{O}\left[\widehat{g}_{\mu \nu}\right]\right\rangle=\frac{\int \mathcal{D} \widehat{g}_{\mu \nu} \mathcal{O}\left[\widehat{g}_{\mu \nu}\right] e^{-S_{\text {grav }}\left[\widehat{g}_{\mu \nu}\right]}}{\int \mathcal{D} \widehat{g}_{\mu \nu} e^{-S_{\text {grav }}\left[\widehat{g}_{\mu \nu}\right]}} .
$$

Here and in the following, indicates the fields that are integrated over. The formal definition $\mathbf{2}$ faces several problems. Some of them are standard problems of the quantization of gauge theories, and some of them are specific to quantum gravity. The latter problems include, for example, the lack of perturbative renormalizability of gravity for $S_{\mathrm{EH}}$ [28-31], the apparent unitarity problems for higher derivative gravity a la Stelle [32-34], and the question whether the integration measure $\mathcal{D} \widehat{g}$ includes a sum over all topologies [35]. The latter question is also an eminent one in lattice gravity (see, e.g., 36-42). Note in this context that a general measure $\mathcal{D} \mu(\widehat{g})$ can always be absorbed with a change of the gravity action in 2 ,

$$
\mathcal{D} \mu(\widehat{g})=\mathcal{D} \widehat{g}_{\mu \nu} e^{-\Delta S_{g r a v}\left[\widehat{g}_{\mu \nu}\right]},
$$

with a potentially nonlocal action $\Delta S_{\text {grav }}$. In the fRG approach, the task of a finite definition of $\mathbf{2}$ and its computation is turned into 
the task of solving a flow equation for the quantum effective action $\Gamma[\bar{g}, \phi]$. Here, $\bar{g}_{\mu \nu}$ is the background or reference metric, and $\phi$ are fluctuation fields, the expectation values of the fluctuation field operators $\hat{\phi}$. The latter includes the fluctuation field $\widehat{h}_{\mu \nu}$ of the metric, $g_{\mu \nu}=g_{\mu \nu}(\bar{g}, h)$, and potential matter fields $\phi_{\text {mat }}$ and auxiliary fields such as the ghosts $c_{\mu}$ of the gauge fixing in gravity,

$$
\phi=\langle\widehat{\phi}\rangle, \quad \phi=\left(h_{\mu \nu}, c_{\mu}, \bar{c}_{\mu}, \phi_{\text {mat }}\right) .
$$

In the case of further gauge fields, one may also use background fields for the gauge fields, which are suppressed here for the sake of convenience. A reparameterization $g_{\mu \nu}(\bar{g}, h) \quad$ seemingly introduces a background-metric dependence of the formulation. This is common to many approaches to quantum gravity due to the necessity of defining metric fluctuations. Accordingly, the question of background independence of the present approach is an eminent one and is discussed later. Here, we only want to mention the most common split between the background metric and the fluctuation field, the linear split,

$$
g_{\mu \nu}=\bar{g}_{\mu \nu}+h_{\mu \nu} \text {. }
$$

This split also underlies most of the results discussed in Section 8. Note that from now on the lowering and raising of indices is done with the background metric $\bar{g}$, if not specified otherwise. Equation 5 emphasizes one specific problem with the background field approach in quantum gravity: while $g_{\mu \nu}$ and $\bar{g}_{\mu \nu}$ are metrics, their difference $h_{\mu \nu}=g_{\mu \nu}-\bar{g}_{\mu \nu}$ is not. Indeed $h_{\mu \nu}$ has no geometrical meaning at all. This is discussed in more detail in Section 3.

\subsection{Gauge Fixing}

In gauge theories such as gravity with the diffeomorphism (gauge) group or the simpler case of non-abelian gauge theories, the practical computation of observables 2 faces the gauge group redundancy in the path integral measure. While this redundancy is a finite-dimensional one within discrete lattice formulations, it is an infinite-dimensional one in functional approaches based on graviton correlation functions. In particular, it prohibits the straightforward definition of the propagator, which is key in most functional approaches.

Therefore, most of the latter approaches require a gauge fixing. (For a brief discussion of gauge-invariant functional approaches, see Section 6.3.) Put differently, we have to choose a parametrization of the theory. Typically, this is done with a linear gauge fixing for the fluctuation field $h_{\mu \nu}$ that carries the metric degrees of freedom,

$$
S_{g f}[\bar{g}, h]=\frac{1}{2 \alpha} \int d^{4} x \sqrt{\bar{g}} \bar{g}^{\mu \nu} F_{\mu} F_{\nu}
$$

A common gauge fixing condition $F_{\mu}$ is given by

$$
F_{\mu}[\bar{g}, h]=\bar{\nabla}^{v} h_{\mu \nu}-\frac{1+\beta}{4} \bar{\nabla}_{\mu} h_{\nu}^{v},
$$

where $\bar{\nabla}$ is the covariant derivative with the background metric $\bar{g}_{\mu \nu}$. The gauge fixing 7 is introduced in the path integral with the Faddeev-Popov trick and the Jacobi determinant of the respective reparameterization. The Faddeev-Popov determinant $\Delta_{\mathrm{FP}}$ can be rewritten in terms of a fermionic path integral with the ghost fields $c_{\mu}$ and $\bar{c}_{\mu}$. The ghost action related to 7 reads

$$
S_{\mathrm{gh}}[\bar{g}, \phi]=\int \mathrm{d}^{4} x \sqrt{\bar{g}} \bar{c}^{\mu} M_{\mu \nu} c^{\nu},
$$

with the Faddeev-Popov operator

$$
M_{\mu \nu}=\bar{\nabla}^{\rho}\left(g_{\mu \nu} \nabla_{\rho}+g_{\rho \nu} \nabla_{\mu}\right)-\frac{1+\beta}{2} \bar{g}^{\sigma \rho} \bar{\nabla}_{\mu} g_{\nu \sigma} \nabla_{\rho} .
$$

Again, $\Delta_{\mathrm{FP}}$ is the covariant derivative with the background metric $\bar{g}_{\mu \nu}$, while $\nabla$ is that with the full metric $g_{\mu \nu}$. Note that $M_{\mu \nu}$ is linear in the fluctuation field $h$. The background metric $\bar{g}_{\mu \nu}$ cannot be avoided, and both gauge fixing and ghost action depend on it. This implies that also the quantum effective action depends on both metrics, the background metric $\bar{g}_{\mu \nu}$ and the full metric $g_{\mu \nu}(\bar{g}, h)$, as we shall see later. Note however that the correlation functions of diffeomorphism-invariant operators and the solutions to the quantum equations of motion do not depend on the gauge fixing. Hence, they are backgroundindependent as explained below.

\subsection{Background Independence}

Background independence of the construction is more than a formal property to aim for. We briefly recollect the standard arguments for background independence in the background field approach to quantum gauge field theories. We first restrict ourselves to pure gravity. Seemingly, background dependence of the path integral is introduced by gauge fixing such as $\mathbf{6}$ and the respective Faddeev-Popov determinant $\Delta_{\mathrm{FP}}$. The latter is the Jacobian of the reparameterization of the path integral in terms of gauge-fixed fields. We emphasize that the gauge fixing should be rather understood as a specific choice of field coordinates in the configuration space that facilitates the integration. The Faddeev-Popov trick is nothing but a convenient way to introduce these coordinates. In any case, it leads us to the expectation values of diffeomorphism-invariant operators defined in $\mathbf{2}$ for pure gravity with a path integral with the gauge-fixed action,

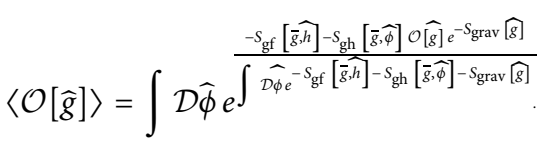

Note that integration over the diffeomorphism group (from the Faddeev-Popov trick) has been factored out in the numerator and denominator. This relies on the diffeomorphism-invariance of $S_{\text {grav }}, \mathcal{O}[\widehat{g}]$, and $\mathcal{D} \widehat{g}$. The full integration measure $\mathcal{D} \widehat{\phi}$ in 10 now also includes the ghost fields, $\widehat{\phi}=\left(\widehat{h}_{\mu \nu}, \widehat{c}_{\mu}, \widehat{\bar{c}}_{\mu}\right)$. Naturally, the right-hand side in $\mathbf{1 0}$ is independent of the background field as the left-hand side trivially is (see 2). This background-metric independence is captured in the Nielsen or split Ward identity derived from taking a $\bar{g}_{\mu \nu}$ derivative of 10. Typically, one also 
subtracts the Dyson-Schwinger equation for $\langle\mathcal{O}\rangle$, which reads schematically

$$
\int \mathcal{D} \widehat{h} \frac{\delta}{\delta \widehat{h}}\left[e^{-S_{\text {gf }}-S_{\text {gh }}} \mathcal{O} e^{-S_{\text {grav }}}\right]=0 .
$$

This leads us to the Nielsen identity for general diffeomorphism-invariant operators $\mathcal{O}[g]$ with

$$
\left\langle\mathcal{O}[\widehat{g}]\left(\frac{\delta}{\delta \bar{g}}-\frac{\delta}{\delta \widehat{h}}\right)\left(S_{\mathrm{gh}}+S_{\mathrm{gf}}\right)[\bar{g}, \widehat{h}]\right\rangle=0
$$

If solving the path integral within approximations, the check of the Nielsen identity $\mathbf{1 2}$ is crucial as it carries the physical background independence.

The identity $\mathbf{1 2}$ constitutes infinitely many relations for diffeomorphism-invariant correlation functions and can be rephrased in terms of derivatives of the effective action. Correlation functions are conveniently derived from the generating functional $Z[\bar{g}, J]$ obtained by adding source terms for the fluctuation fields to the exponent in the path integral,

$$
Z[\bar{g}, J]=\frac{1}{\mathcal{N}} \int \mathcal{D} \widehat{\phi} e^{-S-S_{\mathrm{gf}}-S_{\mathrm{gh}}+\int \mathrm{d}^{4} x \sqrt{\bar{g}} J^{a} \hat{\phi}_{a}},
$$

where $J=\left(J_{h_{\mu \nu}}, J_{c_{\mu}}, J_{\bar{c}_{\mu}}, J_{\text {mat }}\right)$ and the normalization $\mathcal{N}$ is the denominator in 10. Lowering and rising the field indices are done with the metric $\gamma^{a b}$ in field space. (For details, see Supplementary Material.)

In 13, the action $S=S[\bar{g}, \widehat{\phi}]$ is the "classical" action of the gravity-matter system under consideration. The gauge fixing action $S_{\mathrm{gf}}$ and the ghost action $S_{\mathrm{gh}}$ of the full gravity-matter system may include further gauge fixings of gauge fields. Note that for gravity-matter systems, the "classical" action may not be based on the Einstein-Hilbert action of general relativity as discussed before. More generally also, the matter part may not simply be that of a standard renormalizable QFT in the presence of a dynamical metric background.

The generating functional $Z[\bar{g}, J]$, or rather the Schwinger functional $\log [\bar{g}, J]$, generates connected $n$-point correlation functions of the fluctuation field with

$$
\frac{\delta^{n} \log Z[\bar{g}, J]}{\delta J^{a_{1}} \cdots \delta J^{a_{n}}}=\left\langle\widehat{\phi}_{a_{1}} \cdots \widehat{\phi}_{a_{n}}\right\rangle_{\text {con }},
$$

where the indices $a_{i}$ stand for Lorentz and internal indices as well as species of fields. The subscript con $_{\text {in }} \mathbf{1 4}$ indicates the connected part of the correlation function. We have included a factor of $1 / \sqrt{\bar{g}}$ in the definition of the functional derivative (see Supplementary Material). This cancels the $\sqrt{\bar{g}}$ factor in the space-time integral in the source term of 13. If instead, we had used $\sqrt{\widehat{g}}$ in the source term, derivatives with respect to the current $J$ would generate infinite-order correlation functions.

Note that the generating functional 13 can be expressed with the right-hand side of $\mathbf{1 0}$ with the operator $\mathcal{O}=e^{\int \mathrm{d}^{4} x \sqrt{\bar{g}} J^{a} \widehat{\phi}_{a}}$. However, this operator is neither diffeomorphism-invariant nor background-independent. For that reason, it cannot be mapped into a manifestly background-independent form such as $\mathbf{1 0}$. For $J=0$, we have $\mathcal{O}=1$ and $Z=1$, which is trivially background-independent. Accordingly, for $J \neq 0$, the gaugefixed generating functional $Z[\bar{g}, J]$ is background-dependent, as is the effective action $\Gamma[\bar{g}, \phi]$,

$$
e^{-\Gamma[\bar{g}, \phi]}=\frac{1}{\mathcal{N}} \int \mathcal{D} \widehat{\phi} e^{-\left(S+S_{\mathrm{gf}}+S_{\mathrm{gh}}\right)+\int \mathrm{d}^{4} x \sqrt{\bar{g}}\left(\widehat{\phi}_{a}-\phi_{a}\right) \frac{\delta \Gamma}{\delta \phi_{a}}} .
$$

For the relation $\mathbf{1 5}$, we have used that the effective action $\Gamma$ is the Legendre transformation of the Schwinger functional $W[\bar{g}, J]=\log Z[\bar{g}, J]$. This leads to

$$
J^{a}[\bar{g}, \phi]=(-1)^{s_{a}} \frac{\delta \Gamma[\bar{g}, \phi]}{\delta \phi_{a}},
$$

with the fermion number $s_{a}=1$ for fermions and $s_{a}=0$ for bosons. Then, background-independence is achieved on the fluctuation field equations of motion (EoM) for $J[\bar{g}, \phi]=0$. The on-shell vanishing of the currents entails that all diffeomorphism-invariant quantities are backgroundindependent on-shell, and this independence is carried by $\mathbf{1 2}$.

An important consequence of background-independence is the equivalence of the solutions $\bar{g}_{\mathrm{EoM}}[\phi]$ to the fluctuation field EoM and the background field EoM,

$$
\frac{\delta \Gamma\left[\bar{g}_{\mathrm{EoM}}^{\text {fluc }}, \phi\right]}{\delta h_{\mu \nu}}=0 \leftrightarrow \frac{\delta \Gamma\left[\bar{g}_{\mathrm{EoM}}^{\mathrm{back}}, \phi\right]}{\delta \bar{g}_{\mu \nu}}=0,
$$

with

$$
\bar{g}_{\mathrm{EoM}}^{\text {fluc }}=\bar{g}_{\mathrm{EoM}}^{\text {back }}=\bar{g}_{\mathrm{EoM}} \cdot
$$

If the fluctuation EoM holds, the current $J$ is vanishing, and hence, the background EoM is nothing but the Nielsen identity 12. In turn, if the background EoM holds, the current $J$ necessarily vanishes.

\section{FIELD PARAMETRIZATIONS}

So far, we have not specified the relation between the background metric and the full metric $g(\bar{g}, h)$, which defines the role of the fluctuation field $h$. While most of the computations are done within the linear split $\mathbf{5}$, it is worth discussing the general case. This not only allows us to achieve a better understanding of the linear split but also allows us to discuss the challenges for manifestly diffeomorphism-invariant formulations.

The importance of the different splits for the path integral has been already mentioned in the context of the path integral measure (see the introduction of Section 2 around 2). In the flow equation approach to quantum gravity detailed in the next section (Section 4), the discussion of the path integral measure translates into that of the ordering of fluctuations: the fRG approach to quantum gravity is based on a Wilsonian successive integrating out of quantum fluctuations. In its form of a flow equation for the quantum effective action, $\Gamma[\bar{g}, \phi]$ is has a simple form in terms of the full field-dependent fluctuation field propagator $G[\bar{g}, \phi]$ of the theory (see 30). This is the connected part of the two-point function of the fluctuation field, 


$$
G[\bar{g}, \phi](x, y)=\langle\widehat{\phi}(x) \widehat{\phi}(y)\rangle-\phi(x) \phi(y) .
$$

The definition 19 requires a gauge fixing (or reparameterization), as discussed in the previous section. Moreover, the Wilsonian cutoff regularizes the spectrum of the propagator. Consequently, the fRG approach crucially depends on the split of the full metric $g$ into the background metric $\bar{g}$ and the fluctuation field $h$ for two reasons:

i. Ordering of fluctuations: The quantum fluctuations of the fluctuation field $h$ are successively integrated out and are ordered in terms of the background covariant Laplacian. Therefore, the meaning of this ordering depends on the chosen split.

ii. Relevance of higher order correlations: The physics included with higher order correlation functions crucially depends on the chosen split. Thus, a different split orders quantum fluctuations differently. This leads to potentially qualitative differences for the convergence of a given approximation scheme.

In this section, we briefly introduce and discuss the different splits considered so far in the fRG approach to asymptotically safe quantum gravity.

\subsection{Linear Split}

We begin with the standard and simplest split, the linear split (see also 5 ). It is given by

$$
g=\bar{g}+h, \quad \text { with } \quad \mathcal{D} \widehat{g}=\mathcal{D} \widehat{h} .
$$

The Jacobian of this transformation is unity, and the path integral measures agree. As mentioned before, with such a definition, the fluctuation field $h=g-\bar{g}$ is not a metric and has no geometrical meaning in the configuration space of metrics. Still, it is the natural choice as it facilitates explicit computations and the implementation of the quantization of the theory for a given classical action $S_{\text {grav }}$ on the space of metrics. Still, its lack of a geometrical interpretation makes it difficult to discuss the reparameterization invariance of the theory and the consequences of background independence. (For more details, see Section 6.) These intricacies have led to more elaborated splits based on the fiber bundle structure of the configuration space of metrics.

\subsection{Exponential Split}

In recent years, the exponential split has attracted some attention [43-59]. It is given by

$$
g=\bar{g} \exp h, \quad \text { with } \quad \mathcal{D} \widehat{g}=J_{\exp } \mathcal{D} \hat{h} .
$$

The full metric is proportional to the exponential of the fluctuation field $h$ indicating a Lie algebra nature of the fluctuation field $h$. Note that the parametrization 21 restricts the metric $g$, and in particular it does not allow for signature changes. Therefore, it is potentially not a reparameterization of the path integral in terms of integration over all metrics but a definition of another candidate for quantum gravity. Moreover,

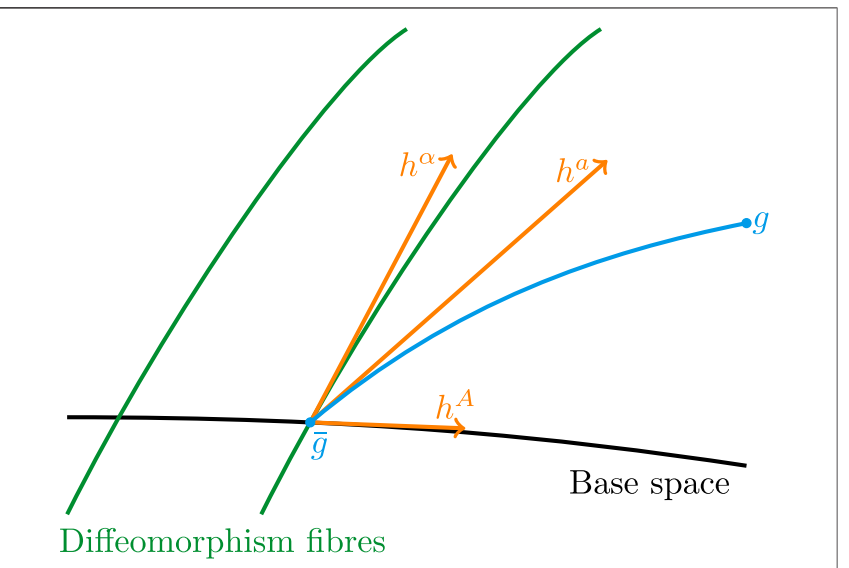

FIGURE 1 | Illustration of the configuration space of metrics with the Vilkovisky connection. The background metric $\bar{g}$ and the full metric $g$ are connected by geodesics. The fluctuation field $h^{a}$ is a tangent vector of these geodesics at the background metric. $h^{A}$ is the projection on the base space, while $h^{\alpha}$ is the projection on the diffeomorphism fiber. The effective action depends only on $h^{A}$ and not on $h^{\alpha}$.

the assumption may change the integration. In summary, it is unclear whether a path integral with the exponential split and the measure $\mathcal{D} \widehat{h}$ describes the same quantum theory as that with the measure $\mathcal{D} \widehat{g}$. This parametrization is also linked to unimodular gravity (see, e.g., [60-66]).

\subsection{Geometrical Split}

We briefly describe the geometrical approach to quantum gravity pioneered by Vilkovisky and DeWitt (see, e.g., [67-70]). In the fRG approach to gravity, it has been discussed in $21,71-75$. It is a general framework, and all parametrizations used in the literature can be understood as different choices for the geometrical structure of the configuration space of metrics $g_{\mu \nu}$. This also allows for a better understanding of the Wilsonian integrating out of qua ntum fluctuations underlying the different splits.

In the linear split, as discussed in Section 3.1, the fluctuation field $h$ neither is a metric nor does it have a geometrical interpretation in the configuration space $\Phi$. In turn, in the geometrical approach, the fluctuation field is constructed such that it has a geometrical meaning. The background metric and the full metric are linked by geodesics with respect to a given connection in the configuration space. The Vilkovisky connection $\Gamma_{\mathrm{V}}$ is a specifically useful one: it is constructed with the demand of maximal orthogonality between the diffeomorphism fiber in the configuration space and the base space. If such disentanglement is achieved, the path integral and the effective action only depend on the propagating degrees of freedom and the gauge redundancies are completely removed. This leads to the following conditions,

$$
\Gamma_{\mathrm{V} B C}^{A}=\Gamma_{\mathrm{g} B C}^{A}, \quad \Gamma_{\mathrm{V} B \gamma}^{A}=0, \quad \Gamma_{\mathrm{V} \beta \gamma}^{A}=0,
$$

where

$$
\Gamma_{\mathrm{g} B C}^{A}=\frac{1}{2} g^{A D}\left(g_{D B, C}+g_{D C, B}+g_{B C, D}\right),
$$


is the Riemannian metric on the quotient space $\Phi / \mathcal{G}$, where $\mathcal{G}$ is the group of diffeomorphisms. This quotient space is labeled with capital Latin letters $A, B, C, \ldots$, while the diffeomorphism fiber is labeled with Greek letters $\alpha, \beta, \gamma, \ldots$. The full space is labeled with small Latin letters $a, b, c, \ldots$.. (For further details on the notation and the setup in the context of RG gravity, see e.g., 72.)

The background metric $\bar{g}$ and the full metric $g$ are connected by a geodesic. With the Vilkovisky connection, the fluctuation field is a tangent vector on this geodesic at the background metric (Gaußian or geodesic normal coordinates). This is illustrated in Figure 1 and leads to

$$
g=\bar{g}+h-\frac{1}{2} \Gamma_{V} h^{2}+\mathcal{O}\left(h^{3}\right), \quad \text { with } \quad \mathcal{D} \widehat{g} \simeq \mathcal{D} \widehat{h} .
$$

The relation between $g$ and $\bar{g}$ is nonpolynomial. Still, the Jacobian does not depend on the fluctuation field, and we have dropped it in $\mathbf{2 4}$. In this setting, it can be shown that the effective action $\Gamma$ only depends on the projection $h^{A}$ of the tangent vector $h^{a}$ onto the base space of the fiber bundle: $\Gamma=\Gamma\left[\bar{g}, h^{A}\right]$. In turn, the projection of $h^{a}$ onto the diffeomorphism fiber, $h^{\alpha}$, drops out. Hence, the effective action is diffeomorphism-invariant as $h^{A}$ is a diffeomorphism scalar. Trivially, an infrared (IR) regularization of the $h^{A}$-path integral is diffeomorphism-invariant.

We close this section with some remarks on the implications of such a geometrical setup for "physical" gauge fixings, linear and exponential splits, and locality. The geometrical construction comes as close as possible to the definition of the configuration space of a gauge theory in terms of "physical" gauge-invariant fields and correlation functions. Such a parameterization is tantamount to a specific gauge fixing as already mentioned in Section 2.2. We may call such a gauge fixing "physical," having in mind that it removes most of the redundancies related to the gauge group, in gravity that related to the diffeomorphism group. Note however that the terminology "physical gauge fixing" is not well-defined and also used differently in other contexts. In non-abelian gauge theories, the projection is unique and singles out the Landau-DeWitt gauge as the "physical" one. In gravity, one is left with a oneparameter family of gauges with the gauge fixing parameter $\beta$ (see 7).

It is worth emphasizing that a gauge fixing condition for the geometrical field (or Gaußian normal field) $h^{a}$ is different from that for the fluctuation field $h$ in the linear split. Only for specific choices of the latter, the maximal disentanglement of the geometrical construction is manifestly obtained. We also remark that the linear split is obtained by using a vanishing connection, hence entirely ignoring the geometrical structure of the configuration space. The exponential split simply uses the Riemannian part $\Gamma_{g}$ of the configuration space, hence ignoring the diffeomorphism group.

Finally, the geometrical construction with the Vilkovisky connection is highly nonlocal in configuration space, one of the ensuing problems being caustics and Gribov copies. This also raises the question of locality in the configuration space and that of momentum locality of the correlation functions of the geometrical fluctuation field $h$. The latter is discussed in detail in
Section 7.4. Both locality issues highlight the challenges for manifest gauge- or diffeomorphism-invariant functional approaches to quantum gravity.

\section{FLOW EQUATION FOR GRAVITY}

With the quantum field theory approach to quantum gravity outlined in the last sections, we are now in the position to discuss the flow equation approach to gravity. (For reviews, see 7-17, and for generic fRG reviews, see 18-27.) As already mentioned in the introduction of Section 3, the fRG approach to gravity is based on a successive integrating out of quantum fluctuations. Typically, this is done with an ordering of quantum fluctuations in momentum space: the regulator introduces a suppression of low-momentum fluctuations below an IR cutoff scale $p^{2} \lesssim k^{2}$, and one RG step with $k \rightarrow k-\Delta k$ relates to the integration of momentum modes $p^{2} \approx k^{2}$. In gravity, the implementation of such a momentum cutoff necessitates the choice of a background metric $\bar{g}$, and the (covariant) momenta are those related to the covariant Laplacian in the background metric, $\Delta_{\bar{g}}$, with the spectral values $p_{\bar{g}}^{2}$.

Remarkably, the flow equation is insensitive to field reparameterizations of quantum gravity discussed in the last section or even physically different formulations: For the derivation, let us assume that a finite generating functional for correlation functions of the fluctuation field is given. In terms of a path integral, this is given by $\mathbf{1 3}$ with an assumed diffeomorphism-invariant regularization and renormalization procedure. More generally, such a finite generating functional is given by its defining property $\mathbf{1 4}$ under the assumption that these correlation functions are finite. Then, the flow equation can be readily derived without the necessity of referring to a specific representation of $Z[\bar{g}, J]$ such as the path integral. (For detailed discussion, see 76.) The correlation functions of $h$ depend on $\bar{g}$, as does the generating functional for $J \neq 0$ via the gauge fixing (see Section 2).

The flow equation for the effective action is derived from the IR regularized generating functional,

$$
Z_{k}[\bar{g}, J]=\exp \left(-\int \mathrm{d}^{4} x \sqrt{\bar{g}} \frac{\delta}{\delta J^{a}} R_{k}^{a b} \frac{\delta}{\delta J^{b}}\right) Z[\bar{g}, J],
$$

with a $\bar{g}$-dependent IR regulator $R_{k}$. Typically, the background dependence enters the regulator via a background Laplacian and background covariant derivatives. In flat space, the eigenvalues of the Laplacian are just momentum squared, $p^{2}$. As already discussed above, the regulator suppresses then IR momentum modes with $p^{2} \leq k^{2}$. In turn, UV momentum modes with $p^{2} \gtrsim k^{2}$ propagate freely, and the generating functional includes all quantum contributions generated by these modes.

It is convenient to write the regulator $R_{k}$ in terms of the classical or quantum dispersion of the field at hand,

$$
R_{k}^{a b}(p)=T_{k}^{a b}(p) r_{k}(x), \quad \text { with } \quad x=\frac{p^{2}}{k^{2}}
$$


where momentum-squared is counted in cutoff units. In these units, the IR regime is given by $x \leqq 1$ and the UV regime by $x \gtrsim 1$. The tensor part $T_{k}^{a b}$ of the regulator is proportional to the classical or quantum dispersion of the field. Classically, it is the second derivative of the action with respect to the fields $\phi^{a}$ and $\phi^{b}$, that is, $\left(S^{(2)}\right)^{a b}(p)$. It carries the kinetic information about the field whose propagation is regularized. In turn, the dimensionless shape function $r_{k}$ specifies how the propagation is regularized. In most cases, the latter part is chosen such that the physical cutoff scales agree for all fields. This is typically achieved with identical (e.g., for several scalar or bosonic fields) or related shape functions (e.g., for scalars and Dirac fermions). It can be shown that such a choice also improves the convergence of generic expansion schemes $[21,77]$. Moreover, $r_{k}$ has to be chosen such that the IR suppression of momentum modes and the UV decay of the regularization are guaranteed. These properties lead to the following asymptotics of the regulator shape function,

$$
\begin{aligned}
& \lim _{x \rightarrow 0} r_{k}(x) \rightarrow \infty, \\
& \lim _{x \rightarrow \infty} r_{k}(x)=0 .
\end{aligned}
$$

The first limit, 27a, guarantees the IR suppression of momentum modes. For example, for a scalar field in $d$ dimensions with a quadratic dispersion $\propto p^{2}$, a regulator shape function $r_{k}(x \rightarrow 0)=1 / x$ introduces a low momentum mass $k^{2}$ for this field. Indeed this is the common choice for the IR limit, but more singular choices work as well. Eq. 27a also entails that for $k \rightarrow \infty$, all momentum modes are suppressed and the theory approaches the UV-scaling regime. For asymptotically free theories, this is the classical theory, and for asymptotically safe theories, this is the nontrivial quantum UV theory.

The second limit, $\mathbf{2 7} \mathbf{b}$, guarantees that the UV behavior of the theory is unchanged by the IR regularization. We shall see below that the limit in $\mathbf{2 7 b}$ has to be approached sufficiently fast. In our example of a scalar field in $d$ dimensions, the regulator shape function has to decay with at least $\min \left((1 / \mathrm{x})^{d / 2} 1 / x\right)$ for rendering the IR flows finite. This is also discussed later in more detail below 39. Note that the latter limit is that of a mass or Callan-Symanzik cutoff. Then, changing $k$ changes a relevant parameter of the theory and hence changes the theory at all scales. Accordingly, the CallanSymanzik cutoff is not a local momentum cutoff. The limit 27b also has another implication: for $k \rightarrow 0$, the limit $\mathbf{2 7 \mathbf { b }}$ holds for all momenta and the cutoff is removed from the theory. We remark that it is precisely this property, which is at stake for the Callan-Symanzik cutoff and similar ones.

Subject to the existence of a finite full generating functional $Z[\bar{g}, J]$, the regularized generating functional $Z_{k}[\bar{g}, J]$ is also finite (and smaller than $Z[\bar{g}, J]$ ). The flow equation for the Schwinger functional $W_{k}[\bar{g}, J]=\log Z_{k}[\bar{g}, J]$ is derived by taking the logarithmic $k$-derivative of $\mathbf{2 5}$. Schematically, this leads us to

$$
\partial_{t} W_{k}[\bar{g}, J]=-\frac{1}{2} \operatorname{Tr}\left(\frac{\delta^{2} W_{k}}{\delta J^{2}}+\frac{\delta W_{k}}{\delta J} \frac{\delta W_{k}}{\delta J}\right) \partial_{t} R_{k},
$$

where the RG-"time" $t$ is defined with $t=\ln k / \Lambda$, and $\Lambda$ is some reference scale $\Lambda$. The trace sums over position space, Lorentz and internal indices, and species of fields. For the sake of a concise presentation, we have suppressed all space-time and internal indices including species of fields. We emphasize that for the explicit form of 28, the order of derivatives is important as $J$ contains fermionic currents.

The term in parenthesis in $\mathbf{2 8}$ is nothing but the full two-point correlation functions of the theory: the first term is the connected part, that is, the scale-dependent propagator $G_{k}[\bar{g}, \phi]$ of the theory (see 19). The second term is simply $\phi^{2}$, the disconnected part. The scale-dependent effective action $\Gamma_{k}[\bar{g}, \phi]$ is defined as the modified Legendre transformation of the Schwinger functional,

$$
\Gamma_{k}[\bar{g}, \phi]=\int \mathrm{d}^{4} x \sqrt{\bar{g}} J^{a} \phi_{a}-W_{k}[\bar{g}, J]-\frac{1}{2} \int \mathrm{d}^{4} x \sqrt{\bar{g}} \phi_{a} R_{k}^{a b} \phi_{b},
$$

where $J=J[\bar{g}, \phi]$ is given by $\mathbf{1 6}$. The source term in (IV) depends on $\sqrt{\bar{g}}$, just as the source term in 13. Otherwise, the Legendre transform would not be linear in the mean field $\phi$. Note also that the classical action of gravity may be unbounded, for example, in the case of the Einstein-Hilbert action. Then, the Legendre transformation is defined on a saddle point.

The flow equation for the effective action $[2,78,79]$ follows straightforwardly from 28 . The part proportional to $\phi^{2}$ is canceled by the flow of the last term in (IV), and the flow of $\Gamma_{k}[\bar{g}, \phi]$ is given by

$$
\partial_{t} \Gamma_{k}[\bar{g}, \phi]=\frac{1}{2} \operatorname{Tr} G_{k}[\bar{g}, \phi] \partial_{t} R_{k}
$$

where $G_{k}[\bar{g}, \phi]$ is the full field-dependent propagator $\delta^{2} W_{k} / \delta J^{2}$, and the trace has been defined below 28. It now contains a relative minus sign for Graßmann-valued fields. With the definition of the Legendre transformation in (IV), the full propagator is given by

$$
G_{k}[\bar{g}, \phi]=\frac{1}{\Gamma_{k}^{(0,2)}[\bar{g}, \phi]+R_{k}} .
$$

The flow equation for the effective action depends on the second derivative of the effective action with respect to the fluctuation fields, $\Gamma_{k}^{(0,2)}[\bar{g}, \phi]$. The flow of the latter is derived from 30, with two derivatives with respect to the fluctuation field $\phi$. This flow depends on itself and the vertices $\Gamma_{k}^{(0,3)}[\bar{g}, \phi]$ and $\Gamma_{k}^{(0,4)}[\bar{g}, \phi]$. This leads to a tower of coupled differential equations for the $n$-point vertices $\Gamma_{k}^{(n, m)}[\bar{g}, \phi]$, which is discussed in more detail in Section 7.1. We use the following notation for derivatives,

$$
\Gamma_{k}^{(n, m)}[\bar{g}, \phi]=\frac{\delta^{n+m} \Gamma_{k}[\bar{g}, \phi]}{\delta \bar{g}^{n} \delta \phi^{m}},
$$

for general functionals of $\bar{g}$ and $\phi$. The functional derivative in 32 includes a factor of $1 / \sqrt{\bar{g}}$ (see Supplementary Material).

The different parameterizations of the metric field, discussed in Section 3, do not influence the flow equation for the effective action 31, and they only differ by their corresponding expansion schemes induced by the relations between metric and fluctuations $(\mathbf{2 0}, \mathbf{2 1}, \mathbf{2 4})$. Still, from the viewpoint of diffeomorphisminvariance, the different parameterizations differ qualitatively. While the geometrical approach with the fluctuation field 24 by construction leads to a diffeomorphism-invariant effective action 
at all cutoff scales, diffeomorphism-invariance is broken in the linear split (20) and the exponential split 21 at a finite cutoff scale.

For all field parameterizations, a diffeomorphism-invariant effective action with one metric $g$ is obtained at vanishing fluctuation graviton field $h=0$,

$$
\Gamma_{k}[g, \varphi]=\left.\Gamma_{k}[g, \varphi]\right|_{h=0}, \quad \text { with } \quad \varphi=\phi(h=0),
$$

the background effective action. Its flow equation is given by $\mathbf{3 0}$, evaluated at vanishing fluctuation field $h=0$,

$$
\partial_{t} \Gamma_{k}[g, \varphi]=\frac{1}{2} \operatorname{Tr} G_{k}[g, \varphi] \partial_{t} R_{k}
$$

Importantly 34 is not closed: the right-hand side depends on $\Gamma_{k}^{(0,2)}$, the two-point function of the fluctuation fields including the fluctuation graviton field $h$, while the left-hand side knows nothing about $h$. Hence, the information about $\delta^{2} \Gamma_{k} / \delta h^{2}$ has to be obtained separately.

\section{BACKGROUND FIELD APPROXIMATION}

The background field approximation, introduced in $[3,80]$ for YangMills theory and gravity, respectively, is the most commonly used approximation in the fRG approach to quantum gravity (see the reviews 7-16). It elevates the diffeomorphism-invariance of the background effective action to that of the full effective action. To that end, we write the full effective action in an expansion about the background effective action in $\mathbf{3 3}$,

$$
\Gamma_{k}[\bar{g}, \phi]=\Gamma_{k}[g, \varphi]+S_{\text {gf }}[\bar{g}, h]+\Delta \Gamma_{k}[\bar{g}, \phi] .
$$

The gauge fixing term $S_{\mathrm{gf}}$ is defined in $\mathbf{6}$ and $\Delta \Gamma_{k}[\bar{g}, \phi=0]=0$. In the background field approximation, the last term in $35 \mathbf{a}$ is assumed to be negligible,

$$
\Delta \Gamma_{k}[\bar{g}, \phi] \approx 0 .
$$

The underlying assumption is that the dynamics of a gauge theory is carried by gauge-invariant fluctuations, while $\Delta \Gamma_{k}$ carries quantum deformations of the gauge fixing procedure and should not drive the dynamics. Then, derivatives with respect to $\bar{g}$ and $h$ agree in the linear split and are related in a simple way in the other parameterizations via 21 and 24.

In the approximation 35 and with the linear split 20, the second derivatives of the effective action with respect to the background metric and the fluctuation field agree at $\phi=0$ up to the gauge fixing term:

$$
\Gamma_{k}^{(0,2)}[\bar{g}, \varphi]=\Gamma_{k}^{(2,0)}[\bar{g}, \varphi]+S_{\mathrm{gf}}^{(0,2)}[\bar{g}, 0] .
$$

Inserting 35 into 30 leads us to a closed and diffeomorphisminvariant flow for the background effective action $\Gamma_{k}[g, \varphi]$.

\subsection{Properties of the Background Approximation}

It is the simple relation $\mathbf{3 6}$ and the manifest diffeomorphisminvariance of the approximation at all cutoff scales that make the background field approximation so attractive. A large amount of the results in asymptotically safe quantum gravity has been obtained in this approximation, and it is still the commonly used approximation in the field. This asks for independent checks of these results and its embedding in systematic expansion schemes that go beyond it. In the present work, we review the fluctuation approach (see Section 7), which includes the correlation functions of the fluctuation graviton field $h$. The results in the background field approximation are qualitatively in line with the results in the fluctuation approach discussed in Section 8. This confirms-in most cases-the underlying assumption 35b. Nonetheless, some words of caution are needed.

Despite its seeming manifest diffeomorphism-invariance, the background field approximation is at odds with diffeomorphisminvariance and background independence. To understand this counterintuitive remark, we recall some features of the background field formalism to standard quantum field theories, for example, the SM and QCD. The introduction of the background field to the gauge fixing allows defining a gaugeinvariant background effective action. It is evident from its introduction that it is an auxiliary symmetry. The background field can even generate gauge-invariant background effective actions in theories that explicitly break gauge-invariance. This is clear from the construction of diffeomorphism-invariant background effective actions in gravity in the presence of a background-covariant momentum regulator. In a gaugeinvariant theory without a cutoff, it can be shown that the physical gauge-invariance of the theory is carried by the fluctuation field in terms of nontrivial Ward- or Slavnov-Taylor identities. The underlying transformations are called quantum gauge/diffeomorphism transformations. This physical symmetry carries over to the auxiliary background gauge invariance via nontrivial Nielsen or split Ward identities. The latter encodes background independence of the theory and is introduced in Section 2.2. The Slavnov-Taylor and Nielsen identities for gravity are discussed in detail in Section 6.

In summary, only if the fluctuation correlation functions satisfy the nontrivial symmetry relations and the Nielsen identities, the auxiliary background gauge-invariance is physical. Then, it carries the underlying symmetry, and we have background independence.

\subsection{Regulator Dependence of the Background Effective Action}

In this section, we first argue that regulator choices within the general class defined with 26 and 27 can be used within the background field approximation to even change the (non-) existence or the nature of an asymptotically safe UV fixed point. This seems to casts some doubts on the reliability of results obtained in the background field approximation. However, we then show that the comparison with fluctuation results and the proper use of Nielsen identities (see Section 6) suffices to further restrict the general class of regulators such that it is adapted to the background field approximation.

The regulator term is the origin of the reliability problems of a naive use of the background field approximation within the fRG 
approach: it generates additional terms in $\Delta \Gamma_{k}[\bar{g}, h]$ in 35a via the background-metric dependence of the regulator. In the background field approximation 35b, this background-metric dependence is elevated to a dynamical one: in the approximation 36, the fluctuation two-point function $\Gamma_{k}^{(0,2)}$ is computed from background-metric derivatives of the (integrated) flow with the exception of the gauge fixing term. These derivatives also hit the regulator. Accordingly, we have added dynamics via the choice of the regulator, and it remains to be proven in each application that this does not change the results qualitatively.

This has been discussed early on at the example of scalar theories and Yang-Mills theory in [81, 82]. In particular, it has been shown that the one-loop $\beta$-function in Yang-Mills theory can be changed from its universal result with regulator choices in the background field approximation. More precisely, it has been shown that for regulators, $R_{k}\left(\bar{\Delta}_{s}\right)$ with spin $s=1$ and spin $s=0$ covariant Laplacians $\bar{\Delta}_{s}=$ $\Delta_{s}(\bar{A})$ the coefficient $Z_{F}$ of the $\operatorname{tr} F^{2}$-term in the effective action runs at one loop as

$$
\left.\frac{\partial_{t} Z_{F}}{Z_{F}}\right|_{1-\text { loop }}=n \beta_{\alpha_{s}, 1-\mathrm{loop}}, \text { for } R_{k}(x \rightarrow 0) \quad \propto \frac{1}{x^{n-1}} .
$$

(For details, refer to 82.) This spoils the universality of the oneloop $\beta$-function in the Yang-Mills theory. If one does not resort to the background field approximation, the correct one-loop $\beta$-function is obtained.

We now discuss the origin of this peculiar behavior. We follow the argument in [83] and for the general case including gravity (refer to $[21,72,73,83]$ ). Simply put, we would like to show that the background effective action at a finite cutoff scale $k$ and in particular in the limit $k \rightarrow \infty$ carries no physics without further restrictions of the regulator. We parameterize the regulator with

$$
R_{k}=\Gamma_{k}^{(0,2)} r_{k}(\bar{\nabla})
$$

(see also 26). Note that in $\mathbf{3 8}$, we have introduced a $\bar{\nabla}$-dependent shape function, which is more general than the $x=\bar{\Delta} / k^{2}$-dependent one (defined in 26). Still we use $x$ in a slight abuse of notation for identifying the UV and IR limits. As already explained around 26, the shape function $r_{k}$ is a free function of the covariant derivative with the limits 27. In particular it has to decay in UV. With the parameterization 38, the flow equation 34 for the background effective action with $\bar{g}=g$ reads

$$
\partial_{t} \Gamma_{k}=\frac{1}{2} \operatorname{Tr} \frac{1}{1+r_{k}} \partial_{t} r_{k}+\frac{1}{2} \operatorname{Tr} \frac{1}{\Gamma_{k}^{(0,2)}} \partial_{t} \Gamma_{k}^{(0,2)} \frac{1}{1+r_{k}} r_{k} .
$$

From the first term on the right-hand side of the flow 39, we deduce that the UV limit of the shape function is constrained: $r_{k}(x \rightarrow \infty) \leq 1 / x^{d+\epsilon}$ with $x=\Delta / k^{2}$, as discussed below 27. In turn, the IR limit $x \rightarrow 0$ of $r_{k}$ can be singular without spoiling the finiteness of 39. In order to obtain a general background effective action, we simply demand that $r_{k}$ solves the differential equation,

$$
\partial_{t} r_{k}=-r_{k} \frac{1}{\Gamma_{k}^{(0,2)}} \partial_{t} \Gamma_{k}^{(0,2)}+2 \partial_{t} Y_{k}
$$

This is a simple differential equation that admits a solution at least locally (in the flow time $t$ ). Note that the UV decay of $r_{k}$ also constrains the UV limit of $Y_{k}$ with $\partial_{t} Y_{k}(x \rightarrow \infty) \leq 1 / x^{d+\epsilon}$. Inserting a shape function $r_{k}$ of $\mathbf{4 0}$ into 39, we arrive at

$$
\partial_{t} \Gamma_{k}=\operatorname{Tr} \partial_{t} Y_{k}(\nabla)
$$

Equation 41 constrains the IR limit of the function $Y_{k}$ : its flow $\partial_{t} Y_{k}$ has to be trace class for rendering the flow of the background effective action finite. If we also assume the trace-class property for $Y_{k}$, the order of $t$-derivative and trace can be swapped.

Apart from these trivial constraints, the choice of $Y_{k}(\nabla)$ is at our disposal. Integrating the flow $\mathbf{4 1}$ from some scale $\Lambda<k$, and taking the UV limit with $k \rightarrow \infty$ we arrive at

$$
\lim _{k \rightarrow \infty} \Gamma_{k}[g, \varphi]=\left[\Gamma_{\Lambda}-\operatorname{Tr} Y_{\Lambda}\right]+\operatorname{Tr} Y_{k}(\nabla) \rightarrow \operatorname{Tr} Y_{k}(\nabla) .
$$

The term $\Gamma_{\Lambda}-\operatorname{Tr} Y_{\Lambda}$ is $k$ - and $\Lambda$-independent, and the latter property follows from RG-consistency: $\partial_{\Lambda} \Gamma_{k} \equiv 0$ for $k \neq \Lambda$ (see, e.g., 84). In the last relation in 42, we have assumed that the effective action is dominated by the UV term $\operatorname{Tr} Y_{k}$. This assumption underlies most fixed-point analyses.

We emphasize that the result 42 is exact and no approximation has been applied. Equation 42 implies that without suitable restrictions on the regulator function $r_{k}$, the flow of the background effective action $\Gamma_{k}[g, \varphi]$ (for large cutoff scales) has no physics content at all. Even at one- and two-loop order in perturbatively renormalizable theories, it does not reproduce universal results without further restrictions on the regulator.

The IR limit with $r_{k \rightarrow 0}=0$ puts a severe restriction onto $r_{k}$, which constrains the integrated flow together with the RG consistency at the initial cutoff scale $\Lambda, \partial_{\Lambda} \Gamma_{k=0}=0$. However, in the UV limit, the restriction

$$
r_{k \rightarrow \infty} \rightarrow \infty \text {, }
$$

does effectively not restrict the UV scaling. The latter is dominated by the UV-relevant operators that satisfy 43 by definition. Note that so far, we have discussed the flow of the background effective action $\Gamma_{k}[g, \varphi]$ without resorting to approximations.

The above issues are already present for the full flow and emphasize the auxiliary nature of the background effective action at $k \neq 0$. In particular, no conclusion can be drawn from its regularity or singular behavior in the UV limit with $k \rightarrow \infty$. This situation is further complicated by the background field approximation. Then, the field dependence that originates from the regulator term is fed back into the flow equation as dynamical contributions. As we have discussed above, these contributions are ambiguous in particular in the UV limit. In conclusion, the background field approximation, while having the appeal of simplicity and seeming diffeomorphism-invariance, has to be applied with great care. To that end, we split the problems discussed above in their physics origin:

(1) Physical diffeomorphism-invariance and background independence are carried by nontrivial Slavnov-Taylor and Nielsen identities of the fluctuation field. 
(2) The background field dependence of the regulator term is potentially dangerous in the UV and has to be separated.

A first step in the resolution of the issues of the background field dependence is to monitor the field-dependence that originates in the regulator. The related equation and discussion in the Yang-Mills theory and gravity can be found in $21,72,73,82,83,85,86$. (For applications to gravity, see also 57, 87-90.) The equation that monitors this dependence is given by

$$
\operatorname{Tr}\left[\frac{\delta \sqrt{\bar{g}} R_{k}}{\delta \bar{g}_{\mu \nu}} \frac{\delta}{\delta R_{k}}\right] \Gamma_{k}[\bar{g}, \phi]=\frac{1}{2} \operatorname{Tr} \frac{\delta \sqrt{\bar{g}} R_{k}}{\delta \bar{g}_{\mu \nu}} G_{k}[\bar{g}, \phi] .
$$

Equation 44 allows to disentangle the background-metric dependence stemming from the regulator from the rest. In the Yang-Mills example from 37, it can be shown that the regulatorfield dependence is responsible for a contribution $(1-n) \beta_{\alpha_{s}, 1-\text { loop }}$. Subtracting the contribution from the regulator-field dependence, the universal result is obtained. Indeed, even without an explicit computation, we can already infer from 44 that the universal 1-loop $\beta$-function of the dimensionless Yang-Mills coupling is achieved for IR regular regulators: the projection of the right-hand side of $\mathbf{4 4}$ on the dimensionless term proportional to $\operatorname{tr} F_{\mu \nu}^{2}$ can only depend on the cutoff scale $k$ in the presence of an additional scale. For IR-regular regulators, such a scale is absent and the $k$-derivative of $\mathbf{4 4}$ vanishes. In turn, IR-singular regulators implicitly introduce a further IR scale, and the $k$-derivative of $\mathbf{4 4}$ does not vanish. This explains the structure of the result in $\mathbf{3 7}$. We emphasize that the modification of the dynamics in the background field approximation via the regulator term is not restricted to IRsingular regulators. The latter fact is a peculiarity of the universal one-loop running of the dimensionless Yang-Mills coupling. In particular, we emphasize that for nonuniversal couplings and theories with dimensionful couplings such as gravity, the flow of 44 does not vanish for IR-regular regulators.

Based on this analysis it has been suggested in 81,82 , that within the background field approximation, the corresponding field-dependence should be subtracted before applying the approximation $\Gamma_{k}^{(0,2)} \simeq \Gamma_{k}^{(2,0)}$ for the right-hand side of the flow. This idea has been picked up by 91-93 for scalar theories, $f(R)$ gravity and gravity matter systems. (For more details, see Section 6.) These works are based on the relation 44, where one derivative with respect to the background is taken. To fully resolve $\Delta \Gamma_{k}$ in 35a, a further field derivative of 44 is needed. Furthermore 44 does not comprise the full difference between $h$ and $\bar{g}$ derivatives. While the background field correlation functions are diffeomorphism-covariant due to background diffeomorphism invariance, the fluctuation correlation functions satisfy difficult Slavnov-Taylor identities. This is well-known and well-studied (though not fully conclusively) in non-abelian gauge theories where one also has access to respective lattice results, for a recent review and related references [27]. In turn, the related analysis, while in high demand, is less advanced in quantum gravity (see also 16, 27). This is detailed in the next section.

\section{SYMMETRY IDENTITIES}

Physical observables are diffeomorphism-invariant and background-independent. The underlying symmetry is dynamical and is solely carried by the dynamical fluctuation fields. It is called quantum diffeomorphism invariance and reads

$$
h_{\mu \nu} \rightarrow h_{\mu \nu}+\mathcal{L}_{\omega}\left(\bar{g}_{\mu \nu}+h_{\mu \nu}\right), \quad \bar{g}_{\mu \nu} \rightarrow \bar{g}_{\mu \nu} .
$$

The background metric triggers an a priori auxiliary symmetry, the background diffeomorphism invariance. It is given by the transformation

$$
h_{\mu \nu} \rightarrow h_{\mu \nu}+\mathcal{L}_{\omega} h_{\mu \nu}, \quad \bar{g}_{\mu \nu} \rightarrow \bar{g}_{\mu \nu}+\mathcal{L}_{\omega} \bar{g}_{\mu \nu} .
$$

Here, $\mathcal{L}_{\omega}$ is the Lie derivative with respect to some vector field $\omega_{\mu}$, which reads for a rank-two tensor

$$
\mathcal{L}_{\omega} T_{\mu \nu}=\omega_{\rho} \bar{\nabla}^{\rho} T_{\mu \nu}+T_{\mu \rho} \bar{\nabla}^{\rho} \omega_{\nu}+T_{\nu \rho} \bar{\nabla}^{\rho} \omega_{\mu} .
$$

Both tranformations, 45 and $\mathbf{4 6}$, generated diffeomorphism transformations on the full metric $g_{\mu \nu}$, so they do not differ on the functional of $g_{\mu \nu}$. Moreover, while $\mathbf{4 6}$ is an auxiliary symmetry, it still comprises the information of the dynamical quantum diffeomorphism symmetry $\mathbf{4 5}$ via the Nielsen identities. The latter carry the background independence of the theory.

Any fRG computation needs to introduce a gauge fixing and a regularization, which both apparently break diffeomorphism invariance and (on-shell) background independence. Thus, it is an important issue in the fRG approach to quantum gravity to discuss how these properties can be preserved in a nonperturbative computation. For each symmetry broken by the cutoff term, we can formulate a nontrivial modified symmetry identity, which captures the cutoff deformation of the underlying symmetry and smoothly approaches the unbroken symmetry identity at vanishing cutoff scale, $k=0$. We now first discuss how the Nielsen identities take care of background independence and afterward discuss quantum diffeomorphism invariance due to the Slavnov-Taylor identities. Note that also in discrete gravity models, the Ward identities play a crucial role (see 94) for a review of tensor models.

\subsection{Background Independence}

As discussed in Section 2.1, we always need to split the full metric into a background metric $\bar{g}$ and a fluctuation field $h$. This split introduces an additional symmetry given by all transformations of the background metric and of the fluctuation field that leave the full metric invariant.

$$
g(\bar{g}, h) \rightarrow g(\bar{g}+\delta \bar{g}, h+\delta h)=g(\bar{g}, h) .
$$

For example, in the linear split 20, we have $\delta \bar{g}=-\delta h$. This symmetry is guaranteeing background independence since we can always find a transformation that changes the background according to our choice. This symmetry is broken off-shell by the gauge fixing and ghost action and further broken by the regularization on- and off-shell. The breaking of the symmetry is described by the Nielsen (or split Ward) identities [95, 96]. They encode the background independence of the physical 
observables and allow us to restore the symmetry at vanishing cutoff.

Let us first discuss the Nielsen identities without the regulator. The Ward identity for the effective action for any symmetry transformation $\mathcal{G}$ is given by

$$
\mathcal{W}=\mathcal{G} \Gamma-\left\langle\mathcal{G}\left(S_{\mathrm{gf}}+S_{\mathrm{gh}}\right)\right\rangle=0,
$$

where $S_{\mathrm{gf}}$ and $S_{\mathrm{gh}}$ are defined as in $\mathbf{6}$ and $\mathbf{8}$. We apply this to the transformation of the metric split $\mathbf{4 8}$ and obtain the Nielsen identity $\mathrm{NI}=0$, with

$$
\mathrm{NI}=\frac{\delta \Gamma}{\delta \bar{g}_{\mu \nu}}-\int\left\langle\frac{\delta \widehat{h}}{\delta \bar{g}_{\mu \nu}}\right\rangle \cdot \frac{\delta \Gamma}{\delta h}-\left\langle\left[\frac{\delta}{\delta \bar{g}_{\mu \nu}}-\int \frac{\delta \hat{h}}{\delta \bar{g}_{\mu \nu}} \cdot \frac{\delta}{\delta \widehat{h}}\right]\left(S_{\mathrm{gf}}+S_{\mathrm{gh}}\right)\right\rangle,
$$

where $h_{\mu \nu}=\left\langle\widehat{h}_{\mu \nu}\right\rangle$, and the fluctuation field is understood as function of the full metric and the background metric $\hat{h}(g, \bar{g})$. For the linear split 20, we have $\delta \widehat{h}_{\rho \sigma}(x) / \delta \bar{g}_{\mu \nu}(y)=1 / \sqrt{\bar{g}} \delta(x-y)$ $1 / 2\left(\delta_{\rho}^{\mu} \delta_{\sigma}^{\nu}+\delta_{\sigma}^{\mu} \delta_{\rho}^{\nu}\right)$ (see Supplementary Material), and thus,

$$
\mathrm{NI}_{\text {lin }}=\frac{\delta \Gamma_{\text {lin }}}{\delta \bar{g}_{\mu \nu}}-\frac{\delta \Gamma_{\text {lin }}}{\delta h_{\mu \nu}}-\left\langle\left[\frac{\delta}{\delta \bar{g}_{\mu \nu}}-\frac{\delta}{\delta \widehat{h}_{\mu \nu}}\right]\left(S_{\mathrm{gf}}+S_{\mathrm{gh}}\right)\right\rangle .
$$

The Nielsen identity for the exponential split 21 resembles 51: there is a nontrivial difference between the background-metric and fluctuation-field derivatives due to the gauge fixing and ghost terms. In 17 , we have pointed out that at $k=0$, a solution of the background EoM is also a solution of the quantum EoM and vice versa. This implies together with $\mathbf{5 1}$ that the expectation value $\left\langle\left[\delta_{\bar{g}}-\delta_{-}\right]\left(S_{\mathrm{gf}}+S_{\mathrm{gh}}\right)\right\rangle$ needs to vanish on-shell. This is indeed nontrivial and does not happen off-shell.

In comparison, for the fully diffeomorphism-invariant Vilkovisky-DeWitt or geometrical effective action with the split given by $\mathbf{2 2}$, the dependence on the gauge fixing action and the ghost action is vanishing, and thus, the Nielsen identity reads

$$
\mathrm{NI}_{\text {geo }}=\frac{\delta \Gamma_{\text {geo }}}{\delta \bar{g}_{\mu \nu}}-\int\left\langle\frac{\delta \widehat{h}}{\delta \bar{g}_{\mu \nu}}\right\rangle \cdot \frac{\delta \Gamma_{\text {geo }}}{\delta h} .
$$

In contradistinction to the linear and exponential split, the $\bar{g}$ and $h$ derivatives are directly related.

The Nielsen identities entail that for all metric splits, the effective action is not a function of the full metric $g$ but depends separately on the background metric $\bar{g}$ and the fluctuation field $h$. Consequently, the effective action has no simple expansion in terms of diffeomorphism-invariant quantities in $g_{\mu \nu}$. Still, the Nielsen identities relate $\bar{g}$ and $h$ derivatives such that on the solution of the Nielsen identities, the effective action carries background independence and only depends on one field.

So far, the analysis has been performed in the absence of the cutoff term, that is, at $k=0$. At finite $k$, the regulator term introduces a further breaking of the split symmetry 48. The Nielsen identities turn into modified Nielsen identities, $\mathrm{mNI}=0$, that read for a general split

$$
\mathrm{mNI}=\mathrm{NI}-\frac{1}{2} \operatorname{Tr} \frac{\delta \sqrt{\bar{g}} R_{k}}{\sqrt{\bar{g}} \delta \bar{g}_{\mu \nu}} G_{k}-\operatorname{Tr} R_{k} G_{k}\left[\frac{\delta}{\delta \phi}\left\langle\frac{\delta \widehat{\phi}}{\delta \bar{g}_{\mu \nu}}\right\rangle\right]
$$

Note that in the last term in 53, only the metric fluctuation $h$ contributes as the other fluctuation fields do not depend on the background metric. Furthermore, in the linear split, the last term is vanishing, and consequently, the $\mathrm{mNI}$ simplifies to

$$
\mathrm{mNI}_{\text {lin }}=\mathrm{NI}_{\text {lin }}-\frac{1}{2} \operatorname{Tr} \frac{\delta \sqrt{\bar{g}} R_{k}}{\sqrt{\bar{g}} \delta \bar{g}_{\mu \nu}} G_{k} .
$$

While some of the properties and consequences of the $\mathrm{mNI}$ are theory-dependent, most of them are generic, and much can be learned about applications in gravity from investigations in general theories: mNIs have been discussed in detail gravity, gauge theories, in scalar theories $[3,15,21,57,72,73,81,82$, 87-93, 98-106].

There is an important qualitative difference between the breaking of the metric split symmetry 48 at finite $k$ and at $k=0$. We have already discussed in Section 2.2 that the Nielsen identity at vanishing cutoff scale, $k=0$, encodes background independence, manifested in the equivalence of the solutions of the background and fluctuation EoMs, 17. At finite cutoff scale, $k \neq 0$, we necessarily have background dependence, as the quantum fluctuations have to be ordered in a specific background. This is also manifest in the missing equivalence of the background and fluctuation EoMs, the respective solutions do not agree,

$$
\frac{\delta \Gamma_{k}\left[\bar{g}_{\mathrm{EoM}}^{\text {fluc }}, 0\right]}{\delta h_{\mu \nu}}=0=\frac{\delta \Gamma_{k}\left[\bar{g}_{\mathrm{EoM}}^{\mathrm{back}}, 0\right]}{\delta \bar{g}_{\mu \nu}}, \quad \bar{g}_{\mathrm{EoM}}^{\text {fluc }} \neq \bar{g}_{\mathrm{EoM}}^{\text {back }} .
$$

(For a detailed discussion, see 97, 106, 107). The difference between the solutions can be parameterized by a term proportional to the regulator $R_{k}$, which is most easily seen in the modified Nielsen identity in the geometric approach, 52 and 53 .

The difference between $\bar{g}_{\mathrm{EoM}}^{\text {fluc }}$ and $\bar{g}_{\mathrm{EoM}}^{\text {back }}$ was explicitly computed in 97, 107 for backgrounds with constant curvature. The ansatz for the background effective action is

$$
\Gamma_{k}[\bar{g}]=\int \mathrm{d}^{4} x \sqrt{\bar{g}} k^{4} f(r)=V \tilde{f}(r),
$$

where $V$ is the space-time volume and $r=\bar{R} / k^{2}$ is the dimensionless background curvature. Thus, the background EoM becomes

$$
\Gamma_{k}^{(\bar{g})}[\bar{g}, 0] \sim r f^{\prime}(r)-2 f(r)=0,
$$

which is displayed in the right panel of Figure 2 at the UV fixed point for different numbers of scalar fields $N_{s}$. The ansatz for the fluctuation one-point function reads

$$
\Gamma_{k}^{\left(h_{\mathrm{tr}}\right)}[\bar{g}, 0]=\int \mathrm{d}^{4} x \sqrt{\bar{g}} k^{3} f_{1}(r)=\frac{V}{k} f_{1}(r)
$$

and thus, the quantum EoM is simply 

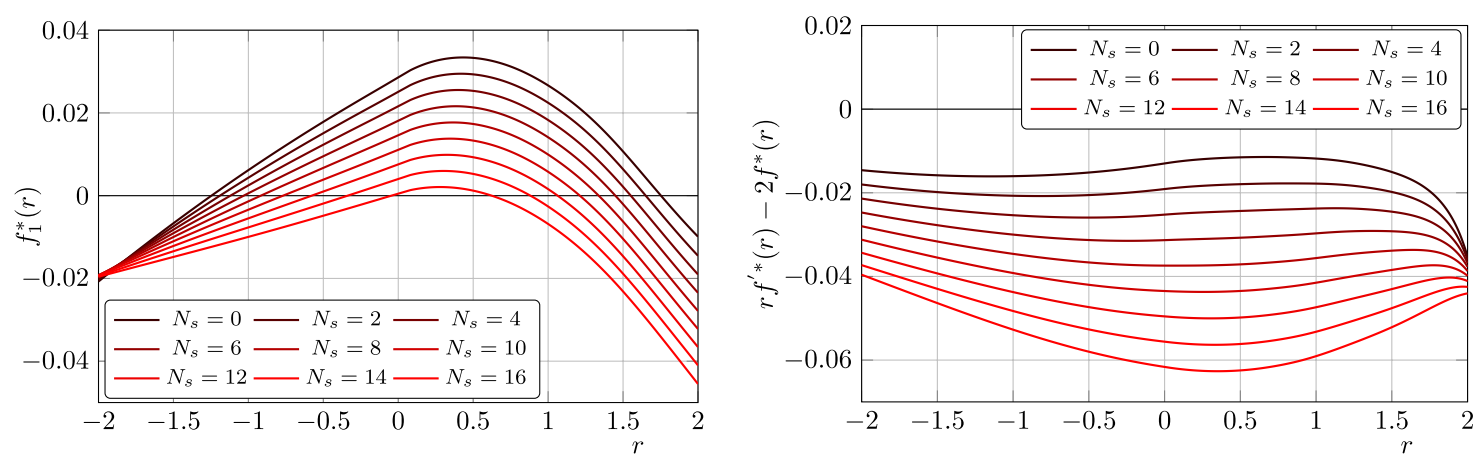

FIGURE 2|Displayed are the potential of the one-point function and the derivate of the background potential for different numbers of scalar fields at the fixed point, as defined in $\mathbf{5 7}$ and $\mathbf{5 9}$. A zero in these functions indicates a solution to the quantum and background EoM, respectively. While the former always has two solutions, a minimum at negative curvature and a maximum at positive curvature, the latter shows no solution at all. The figures are taken from 97.

$$
\Gamma_{k}^{\left(h_{\mathrm{tr}}\right)}[\bar{g}, 0] \sim f_{1}(r)=0 .
$$

This is shown in the left panel of Figure 2 at the UV fixed point for different numbers of scalar fields $N_{s}$.

The background EoM does not display a solution in the whole investigated region, while the quantum EoM has two solutions, a minimum at negative curvature and a maximum at positive curvature. For a larger number of scalar fields, these two solutions merge. However, in this regime, the approximation lacks reliability due to large values of the graviton anomalous dimension. Importantly, Figure $\mathbf{2}$ manifests in explicit computation the difference between the background and quantum EoM, 55. The background EoM was also extensively investigated in the background field approximation with different choices of regulator and parameterization. For example, in 108, the linear split was used and a solution at large negative curvature was found. However, in 109, 110, two further solutions at positive curvature were found due to a different choice of the regulator. A solution at positive curvature was also found in 111 and with the exponential parameterization in 51 .

In 106, a modification of the fRG equation was proposed. There, the effective action was defined as the Legendre transform of a normalized Schwinger functional, $\widehat{W}_{k}[\bar{g}, J]=\log \left(Z_{k}[\bar{g}, J] / Z_{k}[\bar{g}, 0]\right)$. This modification implies that the solutions to the quantum and background EoMs agree even at a finite cutoff scale. This does not imply that the modified effective action is background-independent at finite $k$ since there are differences in the higher order correlation function. However, it allows for constructing improved background field approximations, which might allow resolving some tensions between background and fluctuation results.

\subsection{From BRST to Diffeomorphism Invariance}

While the auxiliary background diffeomorphism invariance 46 remains unbroken, the physical quantum diffeomorphism invariance 45 turns into a BRST symmetry due to the gauge fixing, which is then further broken by the regulator. The related symmetry identities are called (modified) Slavnov-Taylor identities $[(\mathrm{m}) \mathrm{STI}] \quad[112,113]$. They encode physical diffeomorphism invariance. We sketch the main ideas of the derivation and apply them to gravity.

In case of the linear gauge fixing condition 7 , the generator of BRST transformation (or BRST operator) denoted by $\mathfrak{s}$, including the Nakanishi-Lautrup field $\lambda_{\mu}$, is given by

$$
\mathfrak{s}\left(\bar{g}_{\mu \nu}, h_{\mu \nu}, c_{\mu}, \bar{c}_{\mu}, \lambda_{\mu}, \phi_{\text {mat }}\right)=\left(0, \mathcal{L}_{c}\left(\bar{g}_{\mu \nu}+h_{\mu \nu}\right), c_{\rho} \bar{\nabla}^{\rho} c_{\mu}, \lambda_{\mu}, 0, \mathfrak{s} \phi_{\text {mat }}\right) \text {. }
$$

In (B), the vector field $\omega_{\mu}$ in the Lie derivative $\mathbf{4 7}$ is given by the ghost field, $\omega_{\mu}=c_{\mu}$. (For more details on the setup and the condensed notation used below, see 21). The Nakanishi-Lautrup field $\lambda_{\mu}$ transforms trivially under the BRST transformation, $\mathfrak{s} \lambda_{\mu}=0$. The classical gauge-fixed action including the gauge fixing and the ghost action is invariant under this transformation, $\mathfrak{G}\left(S_{\text {grav }}+S_{\text {gf }}+S_{\text {gh }}\right)=0$. Furthermore, $\mathfrak{S}$ is a nilpotent operator with $\mathfrak{S}^{2}=0$.

For the derivation of the STI, we include a source term $Q^{a} \mathfrak{s} \widehat{\phi}_{a}$ for the BRST variations of the fields in the generating functional. The Schwinger functional now reads

$$
e^{W[\bar{g}, \phi, J, Q]}=\int \mathcal{D} \widehat{\phi} e^{-S_{\mathrm{tot}}+\int \mathrm{d}^{4} x \sqrt{\bar{g}}\left(J^{a} \widehat{\phi}_{a}+Q^{a} \widehat{\xi}_{a}\right),}
$$

where $S_{\text {tot }}=S_{\text {grav }}+S_{\text {gf }}+S_{\text {gh }}$. The STI follows from the BRSTinvariance of generating functional,

$$
\int \mathfrak{S}\left(\mathcal{D} \widehat{\phi} e^{-S_{\mathrm{tot}}+\int \mathrm{d}^{4} x \sqrt{\bar{g}}\left(J^{a} \widehat{\phi}_{a}+Q^{a} \xi \widehat{\phi}_{a}\right)}\right)=0 .
$$

The source term $J^{a} \hat{\phi}_{a}$ is the only BRST-variant term. The BRST operator $\&$ commutes with bosonic sources and anticommutes with fermionic sources. This leads us to $\mathfrak{s} J^{a} \widehat{\phi}_{a}=J^{a} \gamma_{a}^{b} \mathfrak{s} \widehat{\phi}_{b}$, where the metric $\gamma_{a}^{b}$ carries the minus sign for the fermionic terms (see Supplementary Material). 


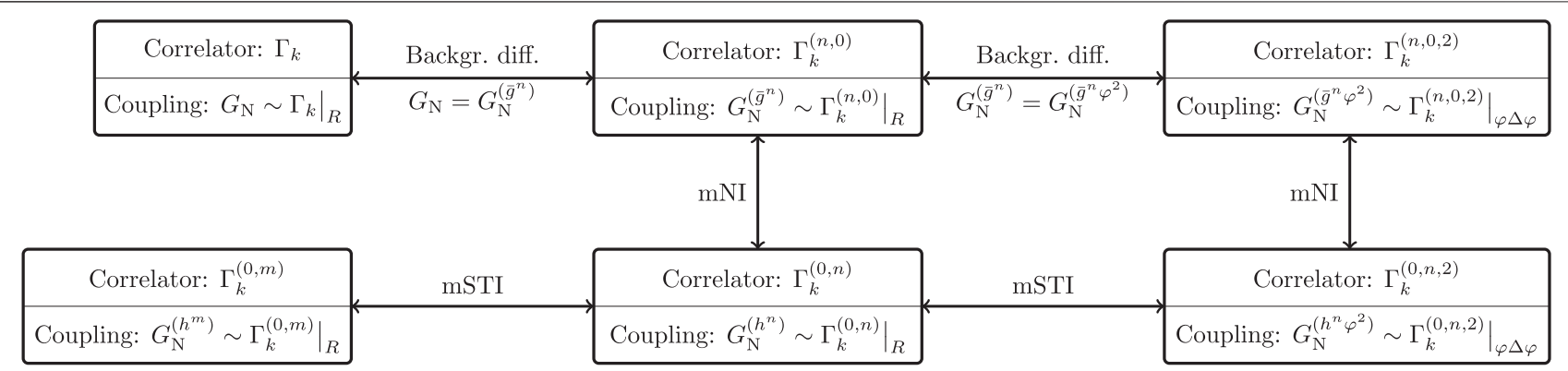

FIGURE 3 | Displayed are the relations between background and fluctuation correlation functions in terms of symmetry identities. The background diffeomorphism symmetry $\mathbf{4 6}$ remains unbroken and trivially connects background correlation functions. The split symmetry (48) is encoded in the modified Nielsen identity ( $\mathrm{mNI}$ ) (53) and relates background correlation functions with fluctuation ones. The quantum diffeomorphism symmetry (45) is described by the modified Slavnov-Taylor identity (mSTI) 65 and relates fluctuation correlation functions. For the purpose of illustration, we have assumed that $\Gamma_{k}=\Gamma_{k}[\bar{g}, h, \varphi]$ depends on the background metric $\bar{g}$, the metric fluctuation $h$, and a scalar field $\varphi$. The notation $\Gamma_{k}^{\left(n_{1}, n_{2}, n_{3}\right)}$ is then defined as in $\mathbf{3 2}$.

With these properties, we obtain the STI for the Schwinger functional,

$$
\begin{aligned}
& \left.\int \mathcal{D} \widehat{\phi} \int \mathrm{d}^{4} x \sqrt{\bar{g}} J^{a} \gamma_{a}^{b}\left(\mathfrak{s} \widehat{\phi}_{b}\right) e^{-S_{\mathrm{tot}}+\int \mathrm{d}^{4} x \sqrt{\bar{g}}\left(J^{a} \widehat{\phi}_{a}+Q^{a} \widehat{\bar{\xi}} a\right.}\right) \\
& =\int \mathrm{d}^{4} x \sqrt{\bar{g}} J^{a} \gamma_{a}^{b} \frac{\delta}{\delta Q^{b}} e^{W[\bar{g}, \phi, J, Q]}=0 .
\end{aligned}
$$

This identity can be re-expressed in terms of the effective action. (See 21 for details.) Here, we just state the result for the STI in the absence of the cutoff term,

$$
\mathrm{STI}=\int \mathrm{d}^{4} x \sqrt{\bar{g}} \frac{\delta \Gamma}{\delta \phi_{a}} \frac{\delta \Gamma}{\delta Q^{a}}=0 .
$$

This equation is known as the quantum-master equation. The BRST variation of the effective action is given by $\delta \Gamma / \delta Q^{a}=\left\langle\xi \widehat{\phi}_{a}\right\rangle$. These variations can be interpreted as generalized vertices of the theory.

Equation 64 encodes diffeomorphism invariance at $k=0$ where the regulator vanishes. At finite cutoff scale, an additional regulator contribution has to be taken into account, and we are led to the mSTI,

$$
\mathrm{mSTI}=\mathrm{STI}-\operatorname{Tr} R_{k} \frac{\delta^{2} \Gamma_{k}}{\delta Q \delta \phi} G_{k}=0 .
$$

Some of the properties of the mSTI are theory-dependent, but most of them are generic: mSTIs in the presence and absence of background fields in gravity and gauge theories have been discussed in detail in $[3,15,21,22,57,72,73,81,82,87-93$, 98-106, 114-132].

In summary, we have three symmetries:

(1) The auxiliary background diffeomorphism invariance 46, which remains unbroken.

(2) The quantum diffeomorphism invariance 45, which describes physical diffeomorphism invariance. It is broken and encoded in the mSTI 65.

(3) The split symmetry 48, which guarantees background independence. It is broken as well and encoded in the mNI 53.
The relations between background and fluctuation correlation functions are summarized in Figure 3. The relation between two fluctuation correlation functions can be expressed either with an mSTI or with a combination of $\mathrm{mNI}$ and background diffeomorphism invariance. However, it should be noted that in a truncated nonperturbative computation these two possibilities of relating fluctuation correlation function do not agree with each other. Nonetheless, it can be used to check the error of the truncation. (See Section 7.1 for more details.)

Last but not least, the flow of $\mathrm{mNI}$ and the mSTI is proportional to itself, respectively. This is conveniently expressed in terms of the flow equation for composite operators, derived in $[21,128,133]$. Schematically, it reads

$$
\partial_{t} \mathcal{O}_{k}[\bar{g}, \phi]=-\frac{1}{2} \operatorname{Tr} G_{k} \partial_{t} R_{k} G_{k} \mathcal{O}_{k}^{(0,2)}[\bar{g}, \phi] .
$$

The operator $\mathcal{O}_{k}^{(0,2)}$ is contracted with $G_{k} \partial_{t} R_{k} G_{k}$ in the trace. The set of composite operators $\mathcal{O}_{k}[\bar{g}, \phi]$ with the flow $\mathbf{6 6}$ includes general correlation functions $Z^{(0, n)}[\bar{g}, J[\bar{g}, \phi]]$ with their disconnected parts as well as more general functions of the field-dependent source such as $J[\bar{g}, \phi]=\Gamma_{k}^{(0,1)}[\bar{g}, \phi]$. In the most general case of a functional with an explicit cutoff dependence, further terms enter 66 (see 21). An educative example is $\Gamma_{k}^{(0,1)}$ : inserting it into 66 leads to the fluctuation field derivative of the flow equation 30. An instructive example for the case of general correlation functions, and the necessity of including the disconnected terms is the full two-point function $G_{\phi_{1} \phi_{2}}+\phi_{1} \phi_{2}$. Equation 66 has been used in Yang-Mills theories for the traced Polyakov loop observables [134] and in gravity for the study of the renormalization and scaling of composite operators [135-138].Importantly, the set of composite operators $\left\{\mathcal{O}_{k}\right\}$ includes modified symmetry identities, that is, Sym $_{k}=\operatorname{mSTI}, \mathrm{mNI}, \ldots$ (see 21 and also 82, 118, 119, 139). Hence, the flow of the symmetry identities reads schematically

$$
\partial_{t} \operatorname{Sym}_{k}[\bar{g}, \phi]=-\frac{1}{2} \operatorname{Tr} G_{k} \partial_{t} R_{k} G_{k} \operatorname{Sym}_{k}^{(0,2)}[\bar{g}, \phi] .
$$

Equation 67 implies that once we have solved these identities at a scale $k$, then the identities are satisfied at all scales. However, 
this only holds for untruncated flows or truncations that are compatible with 67. (More details can be found in Section 7.1.)

\subsection{Challenges for Diffeomorphism-Invariant Flows}

Gauge-invariant approaches to quantum field theories have received much attention over the decades both in perturbation theory and beyond. Such formulations also have met considerable challenges, except for lattice gauge theories that are based on link variables formulated in the gauge group. In turn, perturbation theory and nonperturbative functional approaches are based on correlation functions and in particular on the propagator of the algebra-valued gauge field. (For reviews on lattice approaches to quantum gravity see, e.g., 140-144).

Gauge-invariant functional formulations are based either on gauge-invariant or gauge-covariant variables such as the geometrical formulation, the field strength formulation, or Wilson line formulations similar to lattice gauge theories. Implementations in the flow equation approach range from generalized Polchinski equations with gauge-covariant kernels for the Wilson effective action [145-156] and its recent manifestations [157-159], over the geometrical or Vilkovisky-DeWitt flows for the effective action [21, 71-74], to a recent suggestion for a gauge-invariant flow for the effective action [160-164].

Most of these approaches rely explicitly or implicitly on the definition of projection operators on the subspace of the dynamical degrees of freedom. Typically, this is achieved by a gauge fixing, but the notation of a projection is far more versatile. The appropriate definition of this projection and the respective geometrical structure of the configuration space is at the root of the geometrical construction. This has been discussed in Sections 3.3, 6.2, and 6.3, and we refer to the discussions there. The notable nonlocality of the projections both in field space as well as momentum space is an inherent property of the construction of gauge-invariant subspaces. Consequently, it should be considered an inherent feature of such a construction. This inherent nonlocality may be buried in functional self-consistency relations, but it is present explicitly or implicitly without any doubt.

In any case, the situation calls for self-consistency checks of the final formulations of gauge-invariant or diffeomorphism-invariant flows. This necessity has been discussed already in 165: there the terminology of complete and consistent flows was introduced. The former flows generate all quantum fluctuations from a given classical action, while the latter flows generate a well-defined subset of quantum fluctuations from a given-partial-effective action. A well-known example for the latter is thermal flows, which only generate thermal fluctuations from the full quantum effective action at vanishing temperature. In 165, 166, an important and simple consistency check for flow equations has been suggested: any complete flow equation must generate the complete perturbation theory upon iteration from the given classical action. While one-loop perturbation theory in the fluctuation field is trivially achieved within one-loop exact flow equations, two-loop perturbation theory provides a nontrivial necessary, while not sufficient, consistency check.

These checks for diffeomorphism-invariant fRG approaches have been passed for the Wilsonian approach [145-156] or are trivial for the geometrical effective action approach [21, 71-74]. It is a highly relevant and interesting question how the more recent proposals [158-164] fare in such a self-consistency check. Respective investigations either confirm the completeness of the approaches or may show their consistency, that is, they may integrate out a well-defined subset of quantum fluctuations. Finally, for potentially consistent flows, such an investigation may enable the construction of nontrivial two-loop consistent extensions. We emphasize that such an extension does not simply pass a two-loop test but more importantly allows for two-loop resummed nonperturbative approximations. The latter set of approximations certainly live up to the selfconsistency of other state-of-the-art computations in asymptotically safe quantum gravity, while having the benefit of inherent diffeomorphism invariance.

\section{FLUCTUATION APPROACH}

In the last sections, we have detailed the need for an fRG approach to quantum gravity that goes beyond the background field approximation and that allows satisfying the nontrivial symmetry identities, the mSTI 65 and the mNI 53. For general metrics $g_{\mu \nu}$, this requires to solve the flow equation 30 for the two-field action $\Gamma_{k}[\bar{g}, h]$. It is already a formidable task for the one-field flow in the background field approximation discussed in Section 5. Indeed, already in scalar theories, one has to resort to approximations such as the derivative expansion or the vertex expansion, and this is no different in gravity. As already discussed, while the quantum dynamics of asymptotically safe gravity is generated and carried by the fluctuation correlation functions, it is the diffeomorphism-invariant background effective action $\Gamma[g]$ that allows for a more direct physics interpretation. The latter is extracted from the flow 34 that solely depends on the fluctuation two-point function $\Gamma^{(0,2)}[g, 0]$. The flow of the latter depends on higher order fluctuation correlation functions (see Section 7.1).

This suggests the expansion of the effective action $\Gamma_{k}[\bar{g}, h]$ in a vertex expansion of the fluctuation field $h$. Importantly, the vertex expansion in the fluctuation approach is a systematic approximation scheme, the strength and convergence of which have been shown in many nonperturbative approaches, and most notably in the fRG approach to QCD [167-171]. In the spirit of "toy" theories that can teach us something about technical properties and convergence, we consider non-abelian gauge theories as one of those standard quantum field theories that are as close as it gets to gravity. The vertex expansions fully disentangles the contributions from the background metric $\bar{g}$ and the fluctuation field $h$ and reads for the effective action,

$$
\Gamma_{k}[\bar{g}, \phi]=\sum_{n=0}^{\infty} \frac{1}{n !} \int \Gamma_{k}^{\left(0, \phi_{a_{1}} \ldots \phi_{a_{n}}\right)}[\bar{g}, 0] \cdot \phi_{a_{1}} \ldots \phi_{a_{n}} .
$$

Evidently, if the expansion coefficients $\Gamma_{k}^{\left(0, \phi_{a_{1}} \ldots \phi_{a_{n}}\right)}$ are evaluated for general $\bar{g}$, we have a simple access to the full effective action. For example, if we choose $\bar{g}=\bar{g}_{\text {EoM }}^{\text {fluc }}$, the solution of the fluctuation EoM in 55, we have chosen an on- 
shell expansion point. Accordingly, if we are interested in on-shell physics, only small fluctuations $h$ should be relevant. In turn, if we choose another expansion point, for example, for technical reasons, it is very important to assess whether on-shell physics is in the radius of convergence of the expansion. This will be discussed in more detail in Section 7.2.

\subsection{Hierarchy of Flow Equations}

The background field approach leads to an extended hierarchy of flow equations. We first note that the background flow equation $\partial_{t} \Gamma_{k}[\bar{g}]$ (34) depends on the fluctuation two-point function $\Gamma_{k}^{(0,2)}[\bar{g}, 0]$ in a general background. The knowledge of the latter allows us to determine $\Gamma[\bar{g}]$ and is tantamount to the determination of the full propagator of the theory in a general background. However, the flow of the two-point function $\partial_{t} \Gamma_{k}^{(0,2)}[\bar{g}, 0]$ depends on $\Gamma_{k}^{(0, m)}[\bar{g}, 0]$ with $m=2,3,4$. This continues for higher $n$-point functions and leads to an infinite tower of coupled equations,

$$
\Gamma_{k}^{(0, m)}[\bar{g}, 0]=\mathrm{fRG}_{0, m}\left[\bar{g},\left\{\Gamma_{k}^{(0,2 \leq j \leq m+2)}[\bar{g}, 0]\right\}\right] .
$$

In other words, we need $\Gamma_{k}^{(0,2)}[\bar{g}, \phi]$ for general fluctuation fields for solving the flow equation of the background effective action. For most interacting quantum field theories, the task of resolving the full field dependence of the effective action is beyond reach. Already in scalar theories, one typically resorts to the computation of the full effective potential as well as additional vertices or momentum dependencies. In gravity, the full potential of the background curvature $R$ has been investigated: $f(R)$ as well as potentials of tensor invariants [49, 51, 54, 59, 97, 107-111, 172-183]. Apart from this, as in other theories, explicitly or implicitly, a vertex expansion has been used. This entails a further expansion of $\mathbf{6 9}$ in powers of the background field and leads us to the hierarchy

$$
\Gamma_{k}^{(n, m)}[\bar{g}, 0]=\mathrm{fRG}_{n, m}\left[\bar{g},\left\{\Gamma_{k}^{(i \leq n, 2 \leq j \leq m+2)}[\bar{g}, 0]\right\}\right] .
$$

Equation $\mathbf{7 0}$ is the full hierarchy of integrated flow equations to solve for quantum gravity. While its solution in terms of the vertex expansion has been baptized the fluctuation approach, it simply is the full problem at hand.

Apparently, $\mathbf{7 0}$ constitutes a system of equations for a twofield effective action. However, as discussed in Section 6, background independence at vanishing cutoff, $k=0$, encoded in the Nielsen identities and carried over to the mNIs at finite cutoff scale $k$ turns the effective action into a one-field effective action. In terms of the vertex expansion, this information is given by the $\mathrm{mNI}(\mathbf{5 3})$ for $(n, m)$-point functions,

$$
\Gamma_{k}^{(n, m)}[\bar{g}, h]=\Gamma_{k}^{(n-1, m+1)}[\bar{g}, h]+\operatorname{mNI}_{n, m}\left[\bar{g},\left\{\Gamma^{(i \leq n-1, j \leq m+1)}[\bar{g}, h]\right\}\right] .
$$

This leaves us with two towers of functional relations. While the first one (70) describes the full set of correlation functions, the second one (71) can be used to iteratively solve the tower of mixed fluctuation background correlations on the basis of the

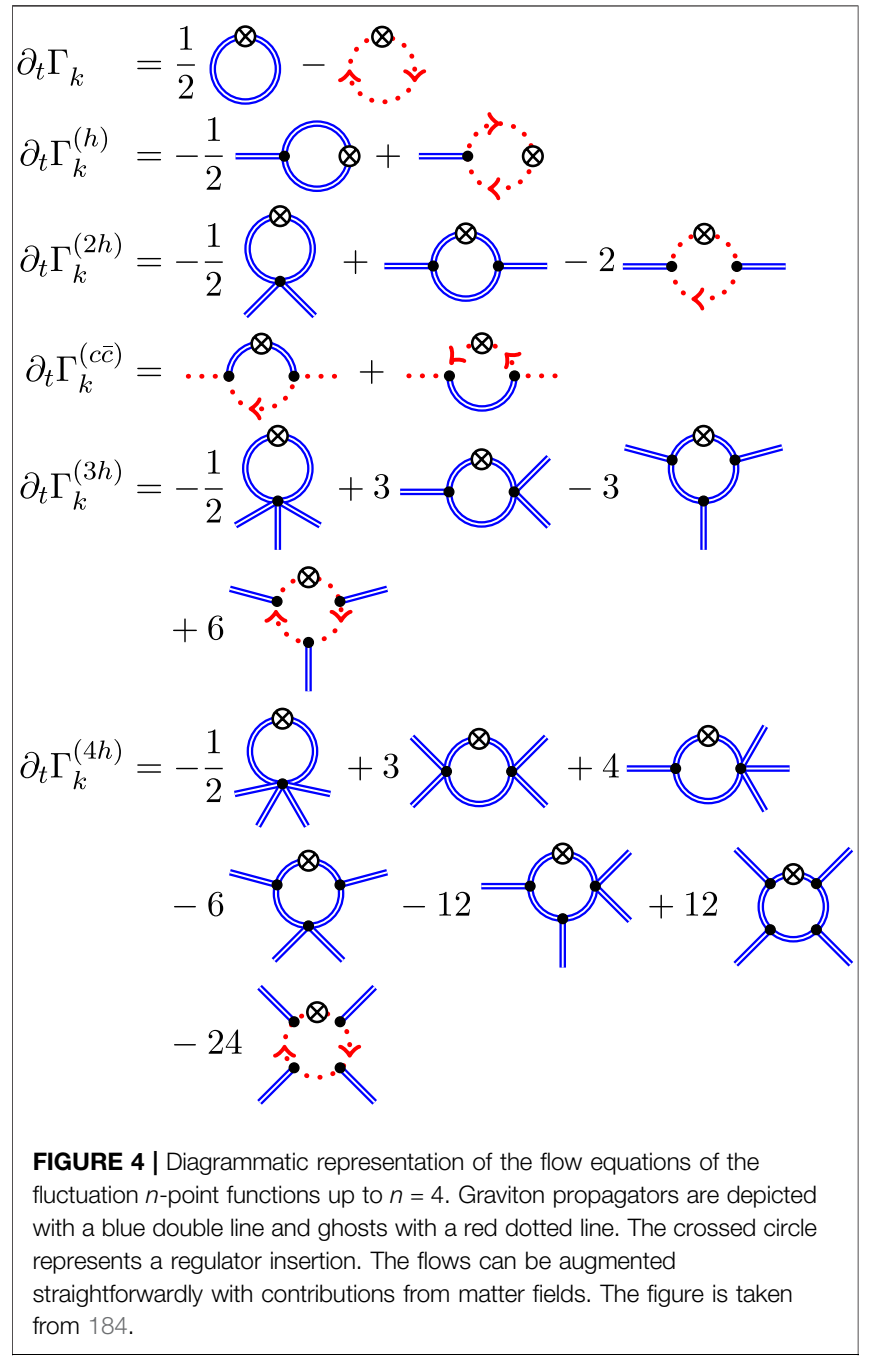

fluctuating correlation functions $\left\{\Gamma^{(0, m)}\right\}$. In both cases, we can solve the system for the higher order correlations of the background on the basis of the lower order correlations. If we use 71 with an iteration starting with the results from the flow equation for $\left\{\Gamma^{(0, m)}\left[\bar{g}_{\mathrm{sp}}, h\right]\right\}$ for a specific background $\bar{g}_{\mathrm{sp}}$, this closure of the system automatically satisfies the NI. Accordingly, any set of fluctuation correlation functions $\left\{\Gamma^{(0, m)}\left[\bar{g}_{\mathrm{sp}}, h\right]\right\}$ can be iteratively extended to a full set of fluctuation background correlation functions in an iterative procedure.

An important feature of the fRG equations is that in the Landau limit of the gauge parameter $\alpha \rightarrow 0$ in $\mathbf{6}$, the flow equations for the transverse vertices $\Gamma_{k, \perp}^{(0, n)}$ are closed: the external legs of the vertices in the flow are transverse due to the transverse projection of the flow, the internal legs are transverse as they are contracted with the transverse propagator. Schematically, this reads for the integrated flows $\mathbf{7 0}$,

$$
\Gamma_{k, \perp}^{(0, n)}=\mathrm{fRG}_{\perp}^{(0, n)}\left[\left\{\Gamma_{k, \perp}^{(0, m)}\right\}\right] .
$$

In other words, the flow equation system of transverse fluctuation correlation functions is closed and determines the 
dynamics of the system. In the fluctuation approach, the transverse system of graviton correlation function has been solved up to the four-graviton vertex [184]. A diagrammatic depiction of the system of flow equations is given in Figure 4, and a description of the respective results can be found in Section 8.

In turn, the flow equation system for longitudinal fluctuation correlation functions is not closed, and the transverse correlation functions $\Gamma_{k, \perp}^{(0, n)}$ feed into it,

$$
\Gamma_{k, L}^{(0, n)}=\mathrm{fRG}_{L}^{(0, n)}\left[\left\{\Gamma_{k, \perp}^{(0, m)}\right\},\left\{\Gamma_{k, L}^{(0, m)}\right\}\right] .
$$

Note that $\left\{\Gamma_{k, L}^{(0, n)}\right\}$ is the complement of the set of purely transverse correlation functions, so it consists of correlation functions with at least one longitudinal leg. On the other hand, the mSTIs are also nontrivial relations for the longitudinal correlation functions in terms of transverse vertices and longitudinal ones. This leads us to the schematic relation,

$$
\Gamma_{k, L}^{(0, n)}=\operatorname{mSTI}^{(0, n)}\left[\left\{\Gamma_{k, \perp}^{(0, m)}\right\},\left\{\Gamma_{k, L}^{(0, m)}\right\}\right] .
$$

(See 185 for non-abelian gauge theories.) In consequence, the mSTIs provide no direct information about the transverse correlation functions without further constraint. In the perturbative regime at large momenta, this additional constraint is given by the uniformity of the vertices. In turn, in strongly correlated regimes such a general constraint is absent. Indeed, one can show that the confinement property in a Yang-Mills theory in a covariant gauge necessitates the absence of uniformity of the vertices at low momenta. (For a detailed discussion in non-abelian gauge theories, see 168.)

Instead, we can simply use $\mathbf{7 4}$ for a given set of transverse correlation functions for constructing a BRST-invariant solution, which signals diffeomorphism invariance. For a given finite set of transverse correlation functions, generically such a solution can be found by integrating the flow (67). However, it may be nonlocal. The existence of BRST-invariant solutions for a general transverse input emphasizes the fact that the derivation of diffeomorphism-consistent solutions is not necessarily the hallmark of a good truncation. However, the comparison of $\mathbf{7 4}$ and $\mathbf{7 3}$ is a further nontrivial constraint on longitudinal correlation functions. Its evaluation is complicated by the fact that the solutions of two different functional relations for the same set of correlation functions do not agree in general in nontrivial truncations. Furthermore, it is very difficult to provide a measure for the closeness of the solutions. (For a related discussion in non-abelian gauge theories, see the recent review [27] and references therein.)

In summary, the evaluation of diffeomorphism invariance and self-consistency constitutes an intricate challenge. One has to utilize all the properties and relations discussed above. This holds for all fRG approaches to quantum gravity and not only to the fluctuation approach: only local BRST-invariant solutions should be considered physical, and the evaluation of locality and BRST invariance or their absence is intricate.

7.2 Flat Expansion Is a Curvature Expansion As briefly mentioned in the introduction of this section, the choice of the background metric is important for the convergence of the vertex expansion. However, an evaluation of the flow equations for $\Gamma_{k}^{(0, n)}[\bar{g}, 0]$ for generic metrics is yet an unresolved technical challenge. Even flows for spherically symmetric backgrounds already pose a formidable technical challenge that has only been solved recently within further approximations that hold for small curvatures [97, 107]. Therefore in most applications, one resorts to a curvature expansion in powers of the curvature. Such an expansion is tantamount to an expansion about the flat background with vanishing curvature,

$$
\bar{g}=1,
$$

the Euclidean analog of the Minkowski metric. This has been baptized the flat expansion. With the flat background (75), Fourier transformations can be performed, and we are led to correlation functions $\Gamma_{k}^{(n, m)}\left(p_{1}, \ldots, p_{n}, p_{n+1} \ldots, p_{n+m}\right)$ in momentum space. This gives access to the powerful techniques of standard quantum field theory that allows solving the flow equations for general vertex functions in momentum space.

This expansion encompasses the standard curvature expansion with the additional benefit that generic covariant momentum dependences are systematically accessible. (For a respective brief discussion, see 184.) To understand this statement, we sketch the curvature expansion of the background field approximation with standard heat kernel techniques and the flat expansion in momentum space. We shall see that both lead to the same flow equations for the expansion coefficients of diffeomorphism-invariant operators. We expand the full one-field effective action in local curvature invariants and covariant derivatives

$$
\Gamma_{k}\left[g_{\mu \nu}\right]=\frac{1}{16 \pi G_{k}} \int \mathrm{d}^{4} x \sqrt{g}\left(2 \Lambda_{k}-R\right)+O\left(R_{\mu \nu \rho \sigma}^{2}, \nabla^{2}\right) .
$$

In 76, the first term on the right-hand side is the Einstein-Hilbert action with a scale-dependent cosmological constant and Newton coupling. The second term includes all other curvature invariants starting with $R^{2}, \ldots$ Covariantderivative terms, schematically given by $\int \sqrt{g} R \nabla^{2} R$ and terms with higher-orders in covariant derivatives $\nabla$, kick in at the next order and beyond. Note that the scale-dependent Einstein-Hilbert action without higher order terms is still a common approximation for the pure gravity sector in particular in many applications to gravity-matter systems. (For gravity-matter systems beyond the Einstein-Hilbert truncation see, e.g., 58, 65, 186-188.)

Similarly to 76, the flow of the background effective action can also be expanded in terms of local curvature invariants and covariant derivatives. This leads us to

$$
\begin{aligned}
\frac{1}{2} \operatorname{Tr} G_{k} \partial_{t} R_{k}\left[g_{\mu \nu}\right]= & \frac{1}{16 \pi} \int \mathrm{d}^{4} x \sqrt{g}\left(2 a_{1, k}-a_{R, k} R\right) \\
& +O\left(R_{\mu \nu \rho \sigma}^{2}, \nabla^{2}\right)
\end{aligned}
$$

with expansion coefficients $a_{\mathcal{O}, k}$ of a given operator $\mathcal{O}$. In particular, we have $a_{1, k}=a_{R^{0}, k}$. By comparing 76 and 77, we arrive at the flow equations 


$$
\partial_{t} \frac{1}{G_{k}}=a_{R, k}, \quad \partial_{t} \frac{\Lambda_{k}}{G_{k}}=a_{1, k} .
$$

Evidently, any complete projection procedure produces the complete set of flow equations of all expansion coefficients $a_{\mathcal{O}, k}$. We emphasize that if the operator basis is overlapping, the flow of the effective action is unique, while the flow of the set of $a_{\mathcal{O}, k}$ is not.

The standard procedure for projecting onto the flow of the cosmological constant and the Newton coupling, as well as that of higher order invariants, is by heat kernel techniques or explicit summation over the spectrum of the covariant Laplacians, in conjunction with the Euler-Maclaurin formula (see the reviews [7-16]). As no other local diffeomorphisminvariant operators are present at this order, the flow of $G_{k}$ and $\Lambda_{k}$ depends only on the given approximation of $\Gamma_{k}^{(0,2)}$ on the right-hand side of the flow. As already indicated, at higher orders of the curvature expansion, more and more invariants are present, and the projections on one single invariant only give unambiguous results if a complete basis of invariants is chosen. In fermionic systems, this is the well-known Fierz ambiguity. (See the review [26] for an extended discussion.) Consequently, at higher orders of the expansion, one typically has to deal with two truncation artifacts: first, we always have to deal with the truncation of $\Gamma_{k}^{(0,2)}$, and second, we have to deal with incomplete bases. We note that only very recently the second order has been mapped out (see 189). This emphasizes that we have to deal with an intricate technical challenge.

Now, we derive the flows in $\mathbf{7 8}$ within the flat expansion scheme. To that end, we note that the only local diffeomorphisminvariant term with no derivatives is the volume term $\mathcal{V}=\int \sqrt{g}$. Moreover, the only local diffeomorphism-invariant term ${ }^{x}$ with two derivatives is the curvature scalar term. This implies that we have a unique projection at the flat expansion point $\mathbf{7 5}$, schematically written as

$$
\begin{aligned}
& -\left.\frac{8 \pi}{\mathcal{V}} \partial_{t} \Gamma_{k}[g]\right|_{g \rightarrow 1}=\partial_{t} \frac{\Lambda_{k}}{G_{k}}=a_{1, k}, \\
& \left.16 \pi \partial_{p^{2}} \partial_{t} \Gamma_{k, \mathrm{TT}}^{(2)}[g]\right|_{g \rightarrow 1}=\partial_{t} \frac{1}{G_{k}}=a_{R, k},
\end{aligned}
$$

where the subscript ${ }_{\text {TT }}$ refers to the projection and normalization on the traceless-transverse part. (More details can be found, e.g., in 184.) Equation $\mathbf{7 9}$ simply is $\mathbf{7 8}$, as the flat expansion scheme is a consistent projection scheme.

This procedure can be extended beyond the set of local diffeomorphism-invariant operators:

(1) Take general derivatives with respect to $h_{\mu \nu}(p)$.

(2) Contract with all possible Lorentz tensor structures.

(3) Take derivatives with respect to momenta.

In particular, apart from all local diffeomorphism-invariant term, the expansion captures general covariant momentum dependences including potential IR-singular terms and topological terms. A diffeomorphism-invariant example for the former is the Polyakov action in two dimensions,

$$
-\frac{1}{96 \pi} \int \mathrm{d}^{2} x \sqrt{g} R \frac{1}{\Delta} R
$$

(see, e.g., 190, 191). IR-singular terms are naturally covered by taking into account full momentum dependences of full vertices or momentum channels of specific tensor structures. This has been used extensively in gauge theories such as QCD not only within the fRG approach but also in other functional approaches based on Dyson-Schwinger equations or $n$-particle irreducible hierarchies.

A relevant example for a topological term in gravity is the Gauß-Bonnet term with the density

$$
E[g]=\frac{1}{32 \pi^{2}}\left(R^{2}-4 R^{\mu \nu} R_{\mu \nu}+R^{\mu \nu \rho \sigma} R_{\mu \nu \rho \sigma}\right) .
$$

Metric variations of the local density $E[g]$ are nonvanishing to all order of metric derivatives. In turn, its space-time integral $\chi[g]=\int \mathrm{d}^{4} x \sqrt{g} E[g]$ is the Euler characteristic of the manifold $M$ with $\chi[g] \in \mathbb{Z}$. Consequently, smooth metric variations of $\chi[g]$ (no change of the geometry) are vanishing. Note however that functional derivatives are distributional and do not fall into the class of smooth variations. Moreover, only the combination of the different curvature-squared invariants in $\mathbf{8 1}$ add up to the Euler characteristic $\chi[g]$. The single terms have a generic metric dependence, and with appropriate projections, we can capture their running coefficients. This is the manifestation of a more generic feature, which is already used in the extraction of anomalies in perturbation theory and anomalous as well as topological terms beyond perturbation theory (see, e.g., [192, 193]).

Below, we outline a cautious approach guided by the works 192, 193 in gauge theories. There, a simple example for a topological invariant is the Pontryagin index in a $U(1)$ theory with the density $1 /\left(32 \pi^{2}\right) F_{\mu \nu} \tilde{F}^{\mu \nu}$, where $\tilde{F}^{\mu \nu}$ is the dual field strength. This density is quadratic in the field and is discussed in Supplementary Material. Analogously to this example, we introduce the Gauß-Bonnet term with a local auxiliary field $\theta(x)$,

$$
\chi[g, \theta]=\int \mathrm{d}^{4} x \sqrt{g} \theta(x) E[g], \quad \chi[g, 1] \in \mathbb{Z} .
$$

The auxiliary field $\theta(x)=\theta_{\text {top }}+\Delta \theta(x)$ can be seen as the local coupling of the Gauß-Bonnet density. Its constant part $\theta_{\text {top }}$ with $\nabla \theta_{\text {top }}=0$ is the topological coupling, while its spacetime-dependent part $\Delta \theta(x)$ is part of the couplings of the local diffeomorphism-invariants quadratic in the curvature. Applying two derivatives with respect to the metric field in momentum space leads us to

$$
\left.\frac{\delta^{2} \chi[g, \theta]}{\delta g_{\mu \nu}(p) \delta g_{\rho \sigma}(q)}\right|_{g=\delta}=\frac{1}{16 \pi^{2}} \mathcal{T}^{\mu \nu \rho \sigma}(p, q) \theta(l) \delta(l+p+q) \text {. }
$$

The tensor structure $\mathcal{T}$ is given by

$$
\begin{aligned}
& \mathcal{T}^{\mu \nu \rho \sigma}=\Pi_{0}\left(\delta^{\mu \nu} \delta^{\rho \sigma}-\delta^{\mu(\sigma} \delta^{\rho)}\right)+\Pi_{2}^{\mu \nu \rho \sigma} \\
& +\delta^{\mu \nu} \Pi_{1}^{\rho \sigma}+\delta^{\rho \sigma} \Pi_{1}^{\mu \nu}-\delta^{\mu(\rho} \Pi_{1}^{\sigma)}-\delta^{\nu(\rho} \Pi_{1}^{\sigma) \mu},
\end{aligned}
$$

where we have defined 


$$
\begin{aligned}
& \Pi_{0}=p^{2} q^{2}-(p \cdot q)^{2}, \\
& \Pi_{1}^{\alpha \beta}=2(p \cdot q) p^{(\alpha} q^{\beta)}-p^{\alpha} p^{\beta} q^{2}-q^{\alpha} q^{\beta} p^{2}, \\
& \Pi_{2}^{\alpha \beta \gamma \delta}=p^{\alpha} p^{\beta} q^{\gamma} q^{\delta}+p^{\gamma} p^{\delta} q^{\alpha} q^{\beta}-p^{\alpha} p^{(\gamma} q^{\delta} q^{\beta}-p^{\beta} p^{(\gamma} q^{\delta} q^{\alpha} .
\end{aligned}
$$

The (local) total derivative property of the Gauß-Bonnet density is reflected in the fact that all $\Pi_{i}$ are vanishing for $l=0$ when momentum conservation implies $p=-q$. Accordingly, with $\theta(x)=\theta_{\text {top }}$ and $\theta(l)=\theta_{\text {top }}(2 \pi)^{4} \delta(l)$, the right-hand side of 83 vanishes. However, by collecting the $\theta$ terms on the lefthand and right-hand side of the flow, one can simply project the flow on the running of the coefficient of the topological term. We emphasize that the vanishing of the flow for constant $\theta$ is analogous to the vanishing of the flow $p^{2} \partial_{t} Z_{\phi}$ for $p^{2}=0$. In conclusion, the present expansion scheme is well-capable and well-suited for describing IR divergent and topological terms.

In summary, the flat expansion allows projecting the flow equation on the flow of all coefficients $a_{\mathcal{O}, k}$ for diffeomorphisminvariant operators of the form

$$
\mathcal{O}=\int_{x} \sqrt{g} f_{\mu_{1} \cdots \mu_{4 n}}\left(\nabla_{1}, \ldots, \nabla_{n}\right) \prod_{i=1}^{n} R_{\mu_{i_{1}} \cdots \mu_{i_{4}}} .
$$

Here, $\nabla_{i}$ acts only on the $i$ th Riemann tensor. In the case of the fluctuation correlation functions $\Gamma_{k}^{(0, n)}[\bar{g}]$, no expansion in curvature invariants is possible, but an expansion in covariant tensor structures is possible, though being even more intricate. In case of the flat expansion, this is done with considering all tensor structures of $\Gamma_{k}^{(0, n)}\left(p_{1}, \ldots, p_{n}\right)$. How this can be done has been worked out in QCD (see, e.g., 167-171), and respective computational tools are provided e.g., by [169, 194, 195] or are in preparation.

The findings of the present section can be summarized as follows:

(1) The flat expansion encompassed the curvature expansion. There is no conceptual difference, and both expansions are expansions about the flat background $\bar{g}=1$.

(2) The expansion point of the curvature or flat expansion is not the solution of the EoM, $\bar{g}_{\mathrm{EoM}}^{\text {fluc }}$, and checks of the convergence of the expansion are in high demand.

(3) The fluctuation approach within the flat vertex expansion resolves the difference between fluctuation and background field. As such it simply improves upon the background field approximation within the curvature expansion without introducing other approximations: fluctuation approach results benchmark that in the background field approximation and provide nontrivial reliability checks for the latter.

There are an increasing number of computations that do not rely on the curvature expansion, for example, 49, 51, 54, $109-111,175-182$ in the background field approximation and 97, 107 in the fluctuation approach. This concludes our discussion of the formal properties of the fluctuation approach.

\subsection{Tensor Structure and Momentum Dependence of Vertices}

In the flat expansion, the vertices $\Gamma^{(n)}=\Gamma^{(0, n)}$ are typically rescaled with the wave function renormalizations $Z_{\phi_{a}}$ to obtain the RG-invariant vertices $\bar{\Gamma}^{(n)}=\bar{\Gamma}^{(0, n)}$

$$
\Gamma_{k}^{\left(\phi_{a_{1} \ldots \phi_{a_{n}}}\right)}(\mathbf{p})=\left(\prod_{i=1}^{n} Z_{\phi_{a_{i}}}^{\frac{1}{2}}\left(p_{i}^{2}\right)\right) \bar{\Gamma}_{k}^{\left(\phi_{a_{1} \ldots \phi_{a_{n}}}\right)}(\mathbf{p}),
$$

where, $\mathbf{p}=\left(p_{1}, \ldots, p_{n}\right)$. The wave function renormalizations can be fully absorbed by a redefinition of the fields $\bar{\phi}_{a}=\sqrt{Z_{\phi_{a}}} \phi_{a}$. The wave function renormalization enter the flow equations only via the anomalous dimensions $\eta_{\phi_{a}}$ defined by

$$
\eta_{\phi_{a}}\left(p^{2}\right)=-\partial_{t} \ln Z_{\phi_{a}}\left(p^{2}\right)
$$

which describes the running of the rescaled fields $\partial_{t} \bar{\phi}_{a} \propto \eta_{\phi} \bar{\phi}_{a}$. The RG-invariant vertices $\bar{\Gamma}_{k}^{(n)}$ are then parameterized with a complete set of tensor structures $\mathcal{T}_{j}$ and respective RG-invariant dressings $\mathcal{A}_{k, j}$

$$
\bar{\Gamma}_{k}^{\left(\phi_{a_{1}} \ldots \phi_{a_{n}}\right)}(\mathbf{p})=\mathcal{A}_{k, j}^{\left(\phi_{a_{1}} \ldots \phi_{a_{n}}\right)}(\mathbf{p}) \mathcal{T}_{j}^{\left(\phi_{a_{1} \ldots \phi_{a_{n}}}\right)}(\mathbf{p} ; \text { couplings }),
$$

where the sum over $j$ is implied. The size of the complete set of tensor structures increases rapidly for higher order vertices. The cutoff-dependent dressings $\mathcal{A}_{k, j}$ capture the overall coupling strength of the respective tensor structure and its momentum dependence.

In most applications to gravity, only the Einstein-Hilbert tensor structures deduced from the curvature scalar and the volume term are taken into account. This leads us to

$$
\begin{gathered}
\mathcal{A}_{k}^{(n)}(\mathbf{p})=G_{n}^{\frac{n}{2}-1}\left(\bar{p}^{2}\right), \\
\mathcal{T}^{\left(\phi_{a_{1}} \ldots \phi_{a_{n}}\right)}=G_{\mathrm{N}} S_{\mathrm{EH}}^{\left(\phi_{a_{1}} \ldots \phi_{a_{n}}\right)}\left(\mathbf{p} ; \Lambda_{n}\right),
\end{gathered}
$$

with the Einstein-Hilbert action $\mathbf{1}$ and the momentumdependent global dressing $\mathcal{A}_{k}^{(n)}$ of the Einstein-Hilbert tensor structure. The prefactor $G_{\mathrm{N}}$ in the definition of the tensor structure leaves the latter independent of $G_{\mathrm{N}}$. The couplings $G_{n}$ and $\Lambda_{n}$ resemble the Newton coupling and the cosmological constant, respectively, for each $n$-point function. They are called avatars of the respective coupling. In C, we have already simplified the momentum dependence of the couplings $G_{n}$ : they only depend on the average momentum $\bar{p}^{2}=\left(p_{1}^{2}+\cdots+p_{n}^{2}\right) / n$. The couplings $G_{n}$ are extracted from the flow of the $n$-point functions at a momentum symmetric point. This definition mimics the definition of momentum-dependent couplings in gauge theories. The dimensionless counterparts of $G_{n}$ and $\Lambda_{n}$ are denoted by $g_{n}=G_{n} k^{2}$ and $\lambda_{n}=\Lambda_{n} / k^{2}$.

For $n=0,1$, we have $\Gamma^{(0, n)}=0$ for a flat background. For $n=2$, we have $G_{n}^{n / 2-1}=G_{2}^{0}=1$, and hence, there is no Newton coupling $G_{2}$ for the two-point function. Instead, $\Gamma_{k}^{(0,2)}$ depends on the graviton mass parameter $\mu=-2 \lambda_{2}$ and the dimensionless wave function renormalization $Z_{h}(p)$ of the fluctuation graviton. We emphasize that the graviton mass parameter $\mu$ should not be understood as a physical mass. Moreover, the graviton is not directly related to an asymptotic state. (For a recent discussion, 


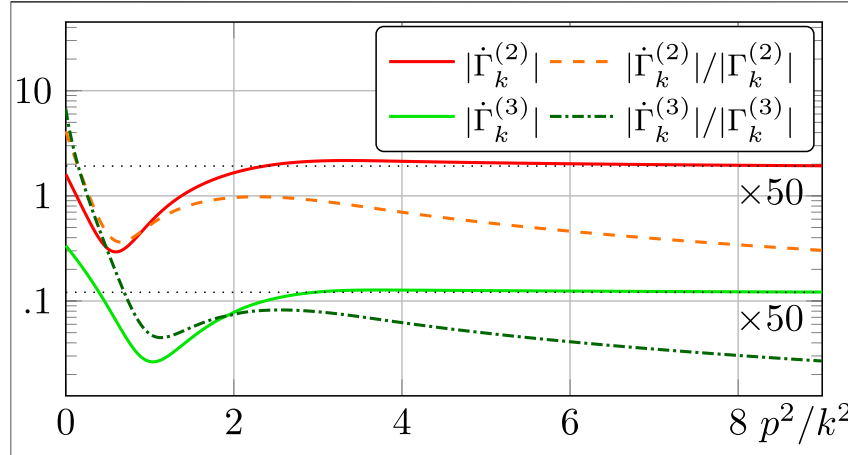

FIGURE 5 | Displayed are the flows of the traceless transverse parts of the graviton two- and three-point functions, $\left|\partial_{t} \Gamma_{k}^{(2)}\right|$ and $\left|\partial_{t} \Gamma_{k}^{(3)}\right|$, as a function of dimensionless momentum. The flows approach constants for large momenta, and they do not grow with $p^{2}$ as expected from a naive counting of momenta. The flows are normalized by the respective $n$-point functions, $\left|\partial_{t} \Gamma_{k}^{(2)}\right| /\left|\Gamma_{k}^{(2)}\right|$ and $\left|\partial_{t} \Gamma_{k}^{(3)}\right| /\left|\Gamma_{k}^{(3)}\right|$. These ratios tend toward zero for large momenta which signals momentum locality. The figure is taken from 197.

see 196 and also the review 16.) All dimensionless couplings are shown in Figure 7 as a function of the cutoff scale on one exemplary UV-IR trajectory.

\subsection{Momentum Locality}

An important property of a physical coarse-graining procedure is momentum locality: it ensures that a coarse-graining step at a given cutoff scale $k$ does not influence the physics at momentum scales $p \gg k$. In 197, it was defined by

$$
\lim _{\frac{p_{i}^{2}}{k^{2}} \rightarrow \infty} \frac{\left|\partial_{t} \Gamma_{k}^{(n, m)}(\mathbf{p})\right|}{\left|\Gamma_{k}^{(n, m)}(\mathbf{p})\right|}=0, \quad \text { with } \quad \mathbf{p}=\left(p_{1}, \ldots, p_{n+m}\right),
$$

In this definition, all momenta $p_{i}$ of the correlation function $\Gamma_{k}^{(n, m)}(\mathbf{p})$ need to be sent to infinity such that there are no trivial cancellations for the momenta of internal propagators. This is, for example, achieved with a symmetric momentum configuration. The norm of the $n$-point function refers to a normalized tensor projection.

The condition $\mathbf{9 1}$ is satisfied by all perturbatively renormalizable local quantum field theories of scalars, fermions, and vector fields (including gauge fields in linear gauges with linear momentum dependences) by trivial counting of the momenta. In turn, nonrenormalizable theories with nontrivial momentum dependences of vertices are easily nonlocal. For example, the scalar field theory with an interaction term of the type $\int \phi^{2}(\partial \phi)^{2}$ does not fulfill 91. Note that this theory has the power counting of Einstein-Hilbert gravity.

Thus, a naïve momentum counting in gravity leads to the conclusion that the coarse graining is not momentum local, neither in Einstein-Hilbert gravity nor in a higher derivative theory of gravity. One needs nontrivial cancellations between diagrams. In 198, such a cancellation was observed for the first time in the transverse traceless part of the graviton two-point function with Einstein-Hilbert vertices. In 197, this was extended to the transverse traceless part of the graviton three-point function. Both cases are displayed in Figure 5. There are three diagrams (plus one ghost diagram) contributing to the flow of the graviton three-point function (Figure 4). The cancellation takes places between all diagrams and holds for all gauge fixing parameters and all momentum configurations of the threepoint function, as long as all external and internal momenta are sent to infinity.

We close this section with the remark that the results in 197 , while highly nontrivial, should be considered to be the first step in a fully conclusive analysis. Most notably, the observed locality does not hold for all tensor structures of the $n$-point functions considered there. In our opinion, this may hint at persistent nonlocalities introduced by the gauge fixing. If this can be solidified in further investigations, this should allow for selecting gauge fixings that make the coarse graining procedure for a given regularization procedure momentum local. Note that while momentum locality of a coarse graining procedure is not a necessary property, it certainly improves the convergence of standard approximation schemes which are typically momentum local. Moreover, if no momentum local coarse graining procedure can be found for a given theory, this casts serious doubts on the interpretation of such a theory as a local quantum field theory.

\section{STATE OF THE ART}

We are now ready to review the state of the art of asymptotically safe quantum gravity within the fluctuation approach. To facilitate accessing the relevance of the different results for the self-consistency of the approach, we start with a brief overview:

UV Fixed Point (Section 8.1): The existence of a UV fixed point with a finite-dimensional critical hypersurface ensures the UV finiteness and predictivity of the theory. With the fluctuation approach, this has been investigated for pure gravity in $73,93,97$, 107, 184, 197-206. The UV fixed point is comparable with results in the background field approximation and thus consolidates these results. Three UV-attractive directions are found associated with $\sqrt{g}, \sqrt{g} R$, and $\sqrt{g} R^{2}$. First signs for apparent convergence within the vertex expansion were found [184].

UV-IR Trajectory (Section 8.2): A UV-IR trajectory allows us to connect to a classical GR regime and IR-SM physics if matter couplings are included. Classical GR regimes are accessed for $\mu \rightarrow 0$ (Gaußian fixed point), $\mu \rightarrow \infty$, and $\mu \rightarrow-1$, where $\mu=$ $-2 \lambda_{2}$ is introduced below (Section 8.3). The case $\mu \rightarrow-1$ was investigated in $73,184,198,199$. In the classical regime, the modified STIs and modified NIs reduce to standard STIs and NIs, which can be solved for small $k$.

Momentum Dependence and Unitarity (Section 8.3): The full momentum dependence, in particular of the propagator, opens a path toward a first investigation of unitarity via spectral reconstructions. The truncations already include the momentum dependence of the graviton two-, three-, and fourpoint functions at the momentum symmetric point [184, 197, 198] as well as the momentum dependence of the graviton-matter three-point vertices [93, 202-204]. The momentum dependence 
has been also used to show the absence of IR divergences in the IR regime $[198,199]$ and to show the absence of $R_{\mu \nu}^{2}$ contributions at the UV fixed point [184].

Curvature Dependence (Section 8.4): The curvature dependence of the correlation functions allows extending the results from a flat background to a generic background. The full curvature dependence of the fluctuation correlation functions contains the information of the diffeomorphism-invariant effective action (Section 7.1). The first steps in this direction in pure gravity and scalar gravity systems have been done in 97 , 107, 206. In 97, 107, the difference of the background and quantum EoM due to the mNI was explicitly computed (see Section 6.1 and Figure 2).

Gravity-Matter Systems (Section 8.5): The aim is to incorporate the SM degrees of freedom in asymptotically safe quantum gravity and eventually to retrodict SM parameters and to constrain beyond the SM physics [207-218]. Minimally and nonminimally coupled gravity-matter systems have been investigated with (partial) fluctuation approach techniques in 53, 83, 93, 97, 187, 202, 204, 219-224. A particularly interesting question is for which matter content the UV fixed point exists. First bounds were computed in 219; however, a qualitative difference between the results in the background field approximation and the fluctuation approach was found [220]. It was shown in 202 that higher order curvature terms are needed to fully address this question. (For gravity-matter systems with higher derivative gravity in the background field approximation, see $58,183,186$.) The investigation in 97 is a first step toward the computational confirmation of the existence of an asymptotically safe fixed point for general gravity matter in the minimally coupled approximation. This opens a path toward reliable stability investigations of fully coupled gravity-matter systems.

Effective Universality (Section 8.6): Last, we discuss the potential close perturbativeness of the UV fixed-point regime of asymptotically safe gravity. This leads us to the concept of effective universality: the so-called avatars of the Newton coupling extracted from different correlation functions may agree up to differences that can be inferred from the modified STIs that relate these couplings [93, 203]. If present, effective universality may have a dynamical origin. The analysis of this intriguing property is also intricate due to truncation artifacts and RG scheme dependences. We close this overview by commenting on the related bimetric approach and hybrids of the background field approximation and the fluctuation approach.

Hybrid approaches: In hybrid approaches, one substitutes part of the fluctuation flow equations with background flow equations $[66,219,225-232]$. In most cases, this concerns the notoriously difficult pure gravity couplings: the derivation of fluctuation flows of pure gravity vertices such as the three- and four-point functions requires a significant computer algebraic effort. In advanced truncations, this is accompanied with numerical loop integrations in every flow step and interpolations of dressing functions with potentially several momentum and angular dependences. In turn, using the background field approximation for these vertices reduces this task to computing the flow of a single background coupling, whose flow equation is known analytically. This considerable reduction makes it chiefly important to construct reliable background field approximation schemes as discussed in Section 5.2

An alternative to the use of the background field approximation for the pure gravity couplings is their identification with matter-gravity couplings. Such an identification implicitly relies on the concept of effective universality discussed in more detail in Section 8.6. There it is discussed that while the full system shows effective universality, it is only maintained if using the pure gravity couplings for the matter-gravity couplings. In turn, effective universality, as well as the compatibility with the full system, is lost if using the matter-gravity couplings as pure gravity ones. This hints at a surprisingly complicated interaction structure in gravity-matter systems whose origin is yet to be understood.

Bimetric approach: The bimetric approach, developed in $100-102,233$, is tantamount to the fluctuation approach reviewed here, as it rests on the distinction between the background metric and the fluctuation field. Technically, fluctuation and background correlation functions are defined in terms of an expansion of the full metric $g_{\mu \nu}=(1+\epsilon) \bar{g}_{\mu \nu}$ with the fluctuation field $h_{\mu \nu}=\epsilon \bar{g}_{\mu v}$. This allows one to order the flow and the effective action in powers of $\epsilon$. The power $\epsilon^{n}$ of the effective action is simply the fluctuation $n$-point function. This reads schematically

$$
\Gamma_{k}[\bar{g}, h]=\sum_{n} \frac{\epsilon^{n}}{n !} \int \Gamma_{k}^{\left(0, h_{\mu_{1} \nu_{1}} \cdots h_{\mu_{n} \nu_{n}}\right)}[\bar{g}, 0] \cdot \bar{g}_{\mu_{1} \nu_{1}} \cdots \bar{g}_{\mu_{n} \nu_{n}},
$$

in analogy to 68. The $\Gamma_{k}^{(0, n)}[\bar{g}, 0]$ have been baptized level- $n$ vertices comprising the respective level-n couplings. The last and most important step concerns the extraction of the correlation function $\Gamma_{k}^{(0, n)}[\bar{g}, 0]$ from $\int \Gamma_{k}^{(0, n)}[\bar{g}, 0] \cdot \bar{g}^{n}$, as the computation of the flow requires the knowledge of the correlation function and not their contractions with metrics. This computation is either done by i) considering an expansion about a specific background such as the flat background, ii) computing the flow of the effective action for a generic metric $\bar{g}$, or iii) assuming a global form of the effective action and simply computing the flow in this closed form. Option i) is the fluctuation approach reviewed here. It is not built on the metric split with $\epsilon$. Option ii) asks for advanced computational heat kernel techniques even within restrictions. These techniques have seen rapid development in the past decade, which may open a path toward their use in ii). Option iii) has been considered so far for level-one couplings. The level-two correlation functions that are required for the right-hand side of the flow equation then have been obtained within a further background field approximation. In summary, the bimetric approach or rather the computational options ii) and iii) offer an alternative approach to compute fluctuation correlation functions that may provide important cross-checks for the results discussed here.

\subsection{Ultraviolet Fixed Point}

The UV fixed point in the fluctuation approach has been discussed in 73, 93, 97, 107, 184, 197-206. This includes work 


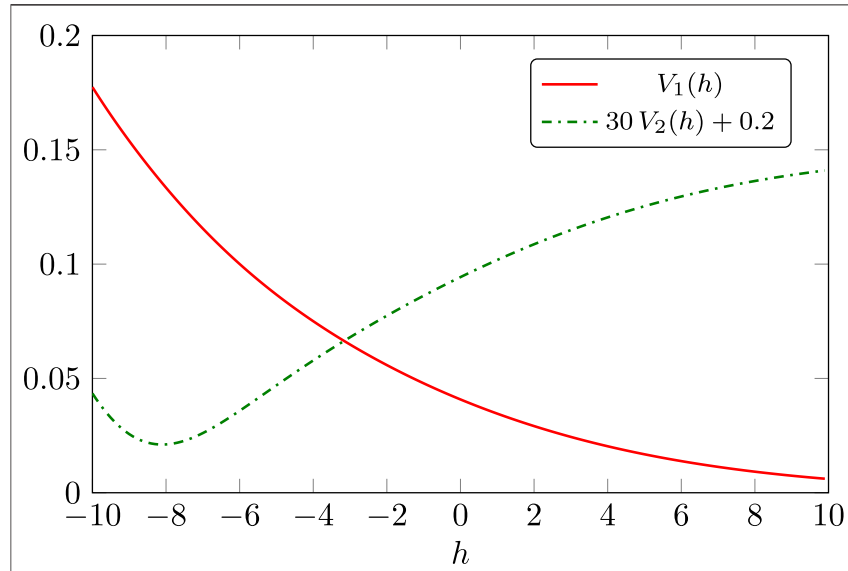

FIGURE 6 | Dimensionless fixed-point fluctuation potentials defined via $V=V_{1}(h)+\operatorname{Tr}\left(h_{\mathrm{TL}}^{2}\right) V_{2}(h)$, where $h$ is the trace part and $h^{\mathrm{TL}}$ is the traceless part of the fluctuation graviton. Note that we rescaled and shifted $V_{2}$, that is, $V_{2}$ is small compared to $V_{1}$ and always negative. The results are taken from 205.

in the vertex expansion about the flat background in pure gravity [184, 197-200] and gravity-matter systems [93, 201-204] as well as work including curvature dependence [97, 107, 206], a fluctuation potential [205] and in the geometrical approach [73]. In 184, the tower of fluctuation correlation functions was implemented until the graviton four-point function. All $n$-point functions were evaluated at the momentum-symmetric point, with external transverse traceless projections. A UV fixed point was found at

$$
\left(\mu^{*}, \lambda_{3}^{*}, \lambda_{4}^{*}, g_{3}^{*}, g_{4}^{*}\right)=(-0.45,0.12,0.028,0.83,0.57),
$$

where $g_{n}$ and $\lambda_{n}$ are the dimensionless Newton coupling and the momentum independent part of the graviton $n$-point function, respectively. (For more details, see 184.) The graviton mass parameter $\mu=-2 \lambda_{2}$ is the momentum-independent part of the graviton two-point function. The critical exponents of the fixed point are given by

$$
\theta_{i}=(4.7,2.0 \pm 3.1 i,-2.9,-8.0)
$$

where a positive sign corresponds to a UV-attractive direction. The three UV-attractive directions were associated with the operators $\sqrt{g}, \sqrt{g} R$, and $\sqrt{g} R^{2}$. In contrast, the operator $\sqrt{g} R_{\mu \nu}^{2}$ is not generated in the present approximation. The latter property was inferred from the momentum dependence of the graviton three- and four-point function (see Section 8.3). Importantly, the first signs of apparent convergence were found in 184.

In Section 7.2, we have shown that the fluctuation approach in the flat expansion improves upon the background field approximation in the curvature expansion (see in particular the discussion at the summary at the end of Section 7.2). Accordingly, the fluctuation results for the UV fixed point detailed above extend and corroborate previous findings in the background field approximation within the curvature expansion.

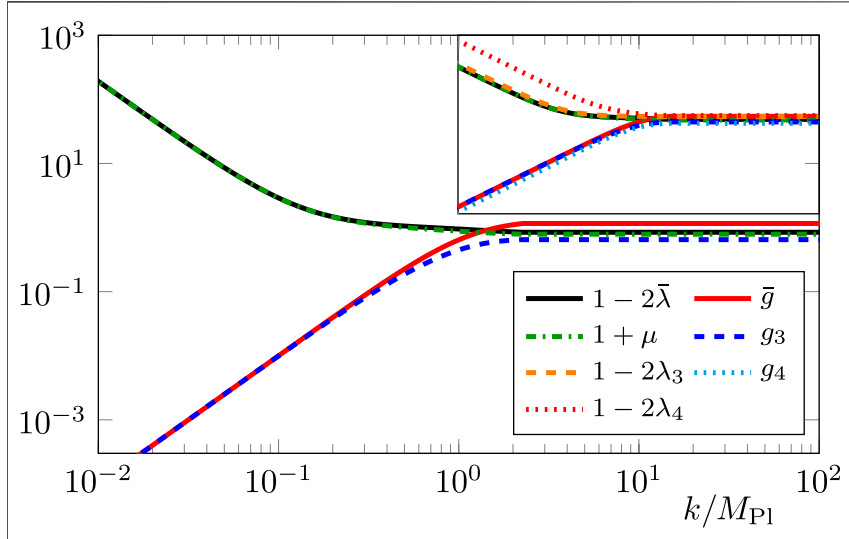

FIGURE 7 | Scale dependence of different fluctuation couplings along a trajectory from the UV fixed point $\mathbf{9 3}$ to the IR. In the IR, the couplings flow according to their canonical running. For small $k, \bar{g}$ and $g_{3}$ as well as $\bar{\lambda}$ and $\mu$ are related via the simplified $\mathrm{NI}(\mathbf{9 5})$. The inset shows the complete set of couplings. The results are taken from 184.
In particular, the results confirm that the latter captures the most important features in pure gravity. For example, the fixed-point value of the cosmological constant in the background field approximation is typically positive, which is comparable with the negative fixed-point value of $\mu$ in $\mathbf{9 3}\left(\mu=-2 \lambda_{2}\right)$. Also, mostly three relevant directions are found in the background field approximation (see the reviews $7-16$ and the very recent paper 189.)

A further extension, within the exponential split, has been investigated in 205. There, the dimensionless fluctuation potential $V$ was approximated with $V=V_{1}(h)+\operatorname{Tr}\left(h_{\mathrm{TL}}^{2}\right) V_{2}(h)$, where $h$ is the trace part and $h_{\mathrm{TL}}$ is the traceless part of the fluctuation graviton. The other graviton modes have been dropped. The results for the potentials $V_{1}$ and $V_{2}$ are displayed in Figure 6.

\subsection{Ultraviolet-Infrared Trajectories}

UV-IR trajectories in the fluctuation approach and hence the phase structure of quantum gravity have been discussed in 73 , 184, 198, 199. In Figure 7, we display a trajectory from the UV fixed point (93) to the IR where all couplings run classically. In the displayed example, the graviton mass parameter runs to infinity, $\mu \rightarrow \infty$. In classical gravity and $\mu>0$, the NIs entail that the cosmological constant is indeed given by $\Lambda=\bar{\Lambda}=-2 \mu k^{2}$ in the limit $k \rightarrow 0$ and can take any negative value. This follows from

$$
\frac{\delta \Gamma_{k}[\bar{g}, h]}{\delta \bar{g}}=\frac{\delta \Gamma_{k}[\bar{g}, h]}{\delta h}, \text { for } \lim _{k \rightarrow 0} \mu \rightarrow \infty \text {. }
$$

Moreover, the background Newton coupling and (all) the fluctuation Newton coupling agree. This can be seen for the dimensionless versions $\bar{\lambda}, \lambda_{2}$ and $\bar{g}, g_{3}$ in Figure 7 . Solving the NIs for the higher couplings corresponds to a fine-tuning problem in terms of choosing an appropriate trajectory. However, a fully diffeomorphism-invariant solution including the higher order 
avatars of the couplings has not been fine-tuned yet (see the inlay in Figure 7).

UV-IR trajectories with $\mu \rightarrow-1$ in the IR have also been investigated in $73,184,198,199$. Those trajectories are technically challenging since $\mu=-1$ corresponds to a pole in the propagator. We emphasize that the NIs and STIs are in this case nontrivial even for classical gravity: the classical effective action is the convex hull of the classical action, the latter not being convex for $\mu<0$. This entails that $\lambda_{2}=-2 \mu$ cannot be identified with the cosmological constant $\lambda=\bar{\lambda}$ even though the sign of the latter must be also positive. Note also that any positive cosmological constant $\Lambda$ can be obtained. The truncation triggered restriction to $\Lambda=0$ at $k=0$ in the background field approximation is lifted. From the physics point of view, these trajectories are appealing since they correspond to a positive cosmological constant in the IR.

\subsection{Momentum Dependence and Unitarity}

The momentum dependence of correlation functions have been discussed in 93, 184, 197-199, 202-204. This momentum dependence encodes the dynamics of the theory and is crucial for the question of unitarity. One of the advantages of the fluctuation approach in the flat vertex expansion is its easy access to the full momentum dependence of fluctuation correlation functions $\Gamma_{k}^{(0, n)}$ for all cutoff scales $k$. These momentum dependences carry the full dynamics of the underlying theory: all other quantities, ranging from the background correlation functions to diffeomorphism-invariant observables $\mathcal{O}[g]$, are built from the correlation functions. The latter observables are defined as expectation values $\mathcal{O}[g]=$ $\mathcal{O}[\bar{g}, h=0]$ of diffeomorphism-invariant operators $\widehat{\mathcal{O}}[\widehat{g}]$ with $\mathcal{O}[\bar{g}, \phi]=\langle\widehat{\mathcal{O}}\rangle$. The $\mathcal{O}[\bar{g}, \phi]$ satisfy the flow equation for the expectation values of composite operators derived in 21 ,

$$
\partial_{t} \mathcal{O}_{k}[g]=-\frac{1}{2} \operatorname{Tr}\left[G_{k} \partial_{t} R_{k} G_{k} \mathcal{O}_{k}^{(0,2)}\right][g],
$$

at vanishing fluctuation field $h=0$. Evidently, the flow 96 solely depends on the fluctuation field propagators and $\mathcal{O}[\bar{g}, \phi]$. (For applications and further investigations of 96 , see 21,128 , 133-135, 137, 138.)

Equation 96 entails in particular that any observable inherits its dynamics from that of the full field and momentum dependence of the fluctuation two-point function, or rather from the momentum dependence of the fluctuation correlation functions $\Gamma_{k}^{(0, n)}$ at a given field expansion point. It is in this sense that the momentum-dependent and RG-invariant vertex dressings $\mathcal{A}^{\left(\phi_{1} \cdots \phi_{n}\right)}(\mathbf{p})$ encode the dynamics of the theory. In particular, the symmetric point dressings $G_{n}(\bar{p})$ carry the meaning of momentum-dependent running couplings similar to those in standard quantum gauge theories and most notably in QCD. (For a detailed discussion in the latter case, see in particular 168,170 .) We emphasize that while in both cases these couplings are neither observables themselves nor even gauge- or diffeomorphism-invariant, they directly encode the dynamics of the theory, and in particular the dominance and/or decoupling of degrees of freedom. If done carefully, they can be also compared to scattering processes related to the respective vertices. (For the SM, see the comparison of the QCD running (vertex) coupling to scattering experiments at accelerators [234].)

Moreover, the resolution of the momentum dependences of $n$-point functions gives at least indirect access to the question of unitarity of asymptotically safe gravity: From the Euclidean data, one can reconstruct Minkowski correlation functions and in particular the graviton spectral functions, both that of the fluctuation graviton and that of the background graviton (more details will be given in 235). Here, we simply comment on the physics content of the graviton spectral functions (see also 236). In this context, we will also use the analogy to the gluon in a nonabelian gauge theory as discussed in 237. (For a recent discussion of the challenges for unitarity in asymptotically safe gravity, see 16.)

To begin with, both the fluctuation graviton and the background graviton two-point functions are not diffeomorphism-invariant. Accordingly, they are not directly related to asymptotic states; even at low energies, gravity is weakly coupled and the theory exhibits a classical momentum and scale dependence (see Figure 7). The latter property suggests that if a Källén-Lehmann spectral representation of the graviton propagators exists, the graviton spectral functions may exhibit a particle-like spectrum for low spectral values. In turn, for large spectral values, we enter the UV fixed point regime, and the physics content of the spectral functions is unclear.

Note however that the same line of arguments would suggest that the gluon spectral function exhibits a particle-type spectral dependence in its perturbative regime for large spectral values. Instead, it can be shown that if a Källén-Lehmann spectral representation exists, the gluon spectral function is negative for large spectral values, and its spectral sum vanishes (Oehme-Zimmermann superconvergence relation). Moreover, it is also negative for small spectral values (see 237). These properties hold for both the fluctuation and the background gluon. Since these properties follow directly from the momentum dependence of the Euclidean correlation functions, we expect similar results for asymptotically safe gravity [235].

In summary, the spectral properties of diffeomorphism- or gauge-variant correlation functions only indirectly mirror the unitarity of the theory. This situation prohibits any direct conclusion of a lack of unitarity from the occurrence of negative parts of spectral functions including negative poles (ghost states). We also emphasize that the latter statement should not be taken as its converse. Of course, the occurrence of negative parts of spectral function requires a thorough investigation of the physics implications and may well be related to the lack of unitarity of the underlying theory. The example of the non-abelian theory simply indicates that this is not necessarily the case. Such an investigation requires the analysis of the spectral properties of diffeomorphism-invariant states. (For a recent discussion of such a setup, see 196.)

The discussion in this section so far emphasizes the importance of the computation of the momentum dependence of correlation functions both for the dynamics of observables and the intricate problem of unitarity. One of the advantages of the fluctuation approach is the direct access to momentumdependent correlation functions with standard quantum field theory methods: 

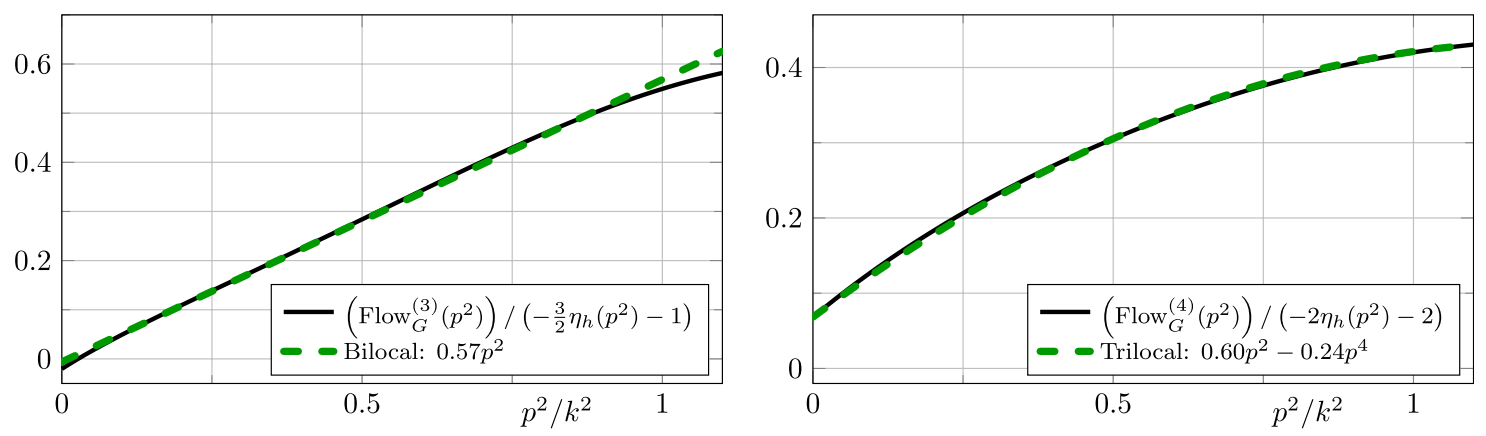

FIGURE 8 | Momentum dependence of the transverse traceless graviton three- and four-point couplings obtained by normalizing the vertex flow with $\left(-\frac{n}{2} \eta_{h}\left(p^{2}\right)-n+2\right)$. The graviton three-point coupling (left panel) is well described with a linear $p^{2}$ function in the momentum range $0 \leq p^{2} \leq k^{2}$. This momentum dependence stems from the $R$ tensor structure. The absence of a $p^{4}$ behavior implies that the $R_{\mu \nu}^{2}$ tensor structure is suppressed. On the other hand, the graviton fourpoint coupling (right panel) shows a clear $p^{4}$ behavior, which is associated with $R^{2}$ tensor structure. The figures are taken from 184 .

In 198, 199, the full momentum dependence of the graviton and ghost propagator was included via the anomalous dimensions. The computation of the momentum dependence was extended to the graviton three- [197] and four-point function [184] as well as to the scalar-graviton [93], the fermion-graviton [204], the gluon-graviton vertex [202], and the ghost-graviton vertex [203]. While only the momenta $0 \leq p^{2} \leq k^{2}$ contribute to the flow, in all these works, the vertices have been computed at the symmetric point for the full momentum range $0 \leq p^{2}<\infty$. This approximation ignores, in particular, the angular dependence of the vertex dressings. While the angular dependence is important for the discussion of the whole phase space of scattering experiments, it is averaged in the flow diagrams due to the angular loop integrations. The reliability of this approximation has been studied at length in QCD. (See 167-171 for detail.) There, it was shown that the above approximation is very accurate in the absence of resonant interaction channels, and so far, no indications have been found for such resonant channels. In conclusion, this analysis provides a nontrivial reliability argument for the approximation described above. Still, for a full reliability check, one has to study extended truncations.

In 184, 200, the momentum dependence was used to disentangle contributions from the couplings of the $R^{2}$ and $R_{\mu \nu}^{2}$ tensor structures. This was done in 200 with derivatives at vanishing momentum, while in 184, the momentum range $0 \leq p^{2} \leq k^{2}$ was considered. Importantly, the transverse traceless graviton three-point function has overlap with $R_{\mu \nu}^{2}$ tensor structures and not with $R^{2}$ tensor structures, while the graviton four-point function has overlap with $R_{\mu \nu}^{2}$ and $R^{2}$ tensor structures. The momentum dependence of the couplings is obtained by normalizing the vertex flows with $\left(-(n / 2) \eta_{h}\left(p^{2}\right)-n+2\right)$. This is displayed in Figure 8. The three-point coupling is well described with a $p^{2}$ behavior. Thus, the $R_{\mu \nu}^{2}$ tensor structure is nontrivially suppressed. The four-point coupling shows a significant $p^{4}$ behavior. Due to the absence of a $p^{4}$ behavior in the three-point coupling, this suggests that they are related to $R^{2}$ tensor structures.

Recently, impressive progress has been made toward momentum-dependent computations in the background field approximation [238-242]. There, the momentum dependence is captured via form factors $W_{k}^{i}$, for example, $\int_{x} R W_{k}^{R}(\Delta) R$ and $\int_{x} C_{\mu \nu \rho \sigma} W_{k}^{C}(\Delta) C^{\mu \nu \rho \sigma}$. This opens a path toward the comparison of the result of these two approaches. This will allow us to quantify the difference between a background field approximation and a fluctuation computation.

\subsection{Curvature Dependence}

The curvature dependence of correlation functions in the fluctuation approach has been discussed in 97, 107, 206. Most results in the fluctuation approach were computed on a flat background $\bar{g}=1$. Results for generic backgrounds can be obtained from an expansion about the flat background. In 206, this was done with covariant heat kernel methods up to the firstorder curvature couplings. Fixed-point values of all first-order curvature couplings were found and their gauge dependence investigated.

A different approach within the fluctuation approach was taken in 97, 107, where the fluctuation correlation functions were computed directly on a generic background with constant curvature. The computation reaches up to the graviton threepoint function and also includes $N_{s}$ scalar fields in 97 . It was found that the curvature dependence of the fluctuation couplings counterbalances the explicit curvature dependence of the respective vertex, making the full vertex approximately curvature independent. This result supports results obtained on a flat background. Furthermore, it was explicitly shown that the background EoM differs from the quantum EoM at the UV fixed point (17). In particular, the background EoM does not have a solution at the UV fixed point, while the quantum EoM has two solutions, a minimum at negative curvature and a maximum at positive curvature, for all $N_{s}$ that are accessible. This is displayed in Figure 2.

\subsection{Gravity-Matter Systems}

A theory of quantum gravity necessarily needs to include matter degrees of freedom to describe our universe. A central question is for which matter content, the UV fixed point exists and if certain types of matter field have a stabilizing or destabilizing effect. Most 
studies have focused on analyzing SM matter fields within the minimally coupled approximation. In this approximation, the matter fields are considered without self-interaction and only couple to gravity via their kinetic term. There are works in the background field approximation [58, 183, 186, 243-249], in the hybrid approach $[53,219]$, and in a full fluctuation computation [93, 97, 201-204]. (For works beyond the minimally coupled approximation, see 47, 50, 83, 162, 186, 188, 210, 220-224, 228, $229,232,250-260$, which also includes scalar-tensor theories and gravitational corrections to the running of matter couplings.)

A major keystone in the stability analysis of gravity-matter systems in the minimally coupled approximation was found in 201, 202. There, it was shown that minimally coupled gravity-matter systems in the Einstein-Hilbert truncation always show a Reuter fixed point as the system can be mapped to a pure gravity system at the level of the path integral. We emphasize that while the explicit computations in 201, 202 are done in the fluctuation approach, the conceptual investigation is general. (For a detailed discussion we refer to 202.) Here, we simply sketch the important steps: In minimally coupled gravity-matter systems, the matter part $S_{\text {mat }}[\bar{g}, \phi]$ of the full action $S=S_{\text {grav }}+S_{\text {mat }}$ is quadratic (or bilinear) in the matter fields. To find the Reuter fixed point, it is sufficient to discuss the UV limit of graviton correlation functions. Consequently, we consider vanishing matter sources, $J_{\text {mat }} \equiv 0$. After performing the Gaußian integration over the matter fluctuation fields $\widehat{\phi}_{\text {mat }}$, the path integral of a minimally coupled matter-gravity system takes the schematic form,

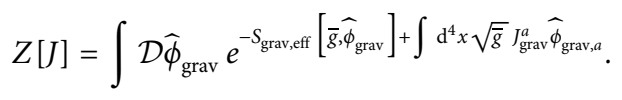

with

$$
S_{\text {grav }, \text { eff }}\left[\bar{g}, \widehat{\phi}_{\text {grav }}\right]=S_{\text {grav }}\left[\bar{g}, \widehat{\phi}_{\text {grav }}\right]+\frac{1}{2} \operatorname{Tr} \log S_{\text {mat }}^{(2)}[\widehat{g}]
$$

Here, the full fluctuation field is split into $\phi=\left(\phi_{\text {grav }}, \phi_{\text {mat }}\right)$ with $\phi_{\text {grav }}=\left(h_{\mu \nu}, c_{\mu}, \bar{c}_{\mu}\right)$, and the hatted field indicate the integration fields. In slight abuse of notation, we wrote $S_{\text {mat }}^{(2)}[\widehat{g}]$ as the second derivative of the matter action with respect to the matter fields. Its argument is $\widehat{g}=\bar{g}+\widehat{h}$, the full metric that is integrated over. Hence, $S_{\text {mat }}^{(2)}$ is a covariant operator and the Trlog contribution is diffeomorphism-invariant.

The form of the generating functional in $\mathbf{9 7}$ is also obtained for UV-complete non minimally coupled matter theories such as Yang-Mills theories. Then, $S_{\text {grav,eff }}[\bar{g}, \widehat{h}]$ is not of the form 98 but carries the full nonperturbative metric-dependent part of the effective action of Yang-Mills theories. The UV completeness within this procedure is required as otherwise the matter path integral cannot be performed. Trivially, minimally coupled systems are UV-complete. A useful analog for the study of the UV stability of minimally coupled gravity-matter systems is many-flavor QCD. There, the role of the graviton is taken by the gluon, and the quark action is bilinear.

The representation 97 emphasizes an intriguing and useful property of the fRG approach to quantum gravity (and beyond): The phase structure and in particular the fixed-point structure of a generic gravity-matter system can be accessed within pure gravity. In particular, all fixed points are accessible within this setup, if a general fixed point effective action $\Gamma_{k}^{*}\left[\bar{g}, \phi_{\text {grav }}\right]$ is considered.

This intriguing property also carries an important intricacy of a generic fixed-point analysis: Seemingly, the parameterization 97 entails that generic gravity-matter systems are UV-stable if the matter part is UV-complete (with the assumption that the Reuter fixed point exists for pure gravity). This conclusion would apply directly to all minimally coupled gravity-matter systems. That this argument falls short can be seen at the example of manyflavor QCD. There, an (f)RG analysis reveals that the QCD $\beta$-function changes its sign for a large enough number of flavors. In the vicinity of this regime, interesting phenomena such as conformal scaling, instabilities, and the Caswell-Banks-Zaks fixed point occur (For fRG literature, see, e.g., 261-264 and references therein.) These findings are backed up by lattice results. The RG analysis in many-flavor QCD solely relies on the marginal operator $\operatorname{tr} F_{\mu \nu}^{2}$. The quantum corrections from the integrating out of the quark fluctuations are proportional to

$$
N_{\mathrm{f}} \operatorname{tr} F_{\mu \nu}^{2} \log \frac{F_{\mu \nu}^{2}}{k^{4}},
$$

where $N_{\mathrm{f}}$ is the number of flavors. The analogous operators in gravity are the curvature-squared operators $R^{2}, R_{\mu \nu}^{2}$, and $R_{\mu \nu \rho \sigma}^{2}$. The respective operators including matter quantum fluctuations are

$$
N_{\text {mat }} \sqrt{g} R^{2} \log \frac{R}{k^{2}}
$$

and similar ones for $R_{\mu \nu}^{2}$, and $R_{\mu \nu \rho \sigma}^{2}$ and also covariant derivatives. Here, $N_{\text {mat }}$ is the weighted sum over all species and flavors of matter fields.

The logarithmic RG running of the marginal operator $\operatorname{tr} F_{\mu \nu}^{2}$ in QCD or $R^{2}$ in gravity necessarily triggers a field dependence of its coefficient as displayed in 99 and 100, respectively. In conclusion, the distinctive property of marginal operators is the inherent field dependence of the quantum corrections. In turn, the coefficients of (local) relevant and irrelevant operators are only scaledependent. While the latter by definition are not important for a fixed-point analysis, the coefficients of the former ones, if present, can be readily absorbed in the respective pure gauge theory (or gravity) couplings. In the present example of manyflavor QCD, relevant operators are indeed absent. In gravity, this applies to the terms in the Einstein-Hilbert action, that is, the curvature term and the cosmological constant term.

In summary, from the perspective of the Yang-Mills system with the generating functional similar to that in $\mathbf{9 8}$, the marginal operator 99 introduces a new UV marginal (and hence physical) parameter $N_{\mathrm{f}}$ that cannot be absorbed in the Yang-Mills coupling. In gravity, this applies to the coefficients of the marginal operators $R^{2}, R_{\mu \nu}^{2}$, and $R_{\mu \nu \rho \sigma}^{2}$. Thus, also here, the flavor number $N_{\text {mat }}$ of a given matter field is a physical parameter. However, its relevance for the fixed-point analysis originates solely from the $N_{\text {mat }}$-dependent coefficients of the marginal operators $R^{2}, R_{\mu \nu}^{2}$, and $R_{\mu \nu \rho \sigma}^{2}$. In contrast, $N_{\text {mat }}$ of the relevant operators in the 
Einstein-Hilbert action is not relevant for the fixed-point analysis. In particular, it cannot trigger instabilities.

The above properties imply that a fixed-point analysis of a given system within a truncation of the (f)RG flows that does not include the flows of the marginal operators should exhibit the respective fixed-point structure of the pure gravity system in the same truncation. In particular, this casts some doubt on any instability findings in the full truncation, if this instability survives in the absence of the marginal operators.

As an example of this statement, we consider now a minimally coupled gravity-matter system in the Einstein-Hilbert truncation. Without truncation, these systems have the path integral representation $\mathbf{9 7}$ with $\mathbf{9 8}$. The Einstein-Hilbert truncation reduces $S_{\text {grav, eff }}$ in $\mathbf{9 8}$ to

$$
\begin{aligned}
& S_{\text {grav }, \text { eff }}\left[\bar{g}, \hat{\phi}_{\text {grav }}\right] \rightarrow S_{\mathrm{EH}}[g]+S_{\mathrm{gf}}[\bar{g}, \widehat{h}]+S_{\mathrm{gh}}\left[\bar{g}, \hat{\phi}_{\text {grav }}\right] \\
& +\left.\frac{1}{2} \operatorname{Tr} \log S_{\text {mat }}^{(2)}[\hat{g}]\right|_{R, \Lambda},
\end{aligned}
$$

where the subscript $\left.\right|_{R, \Lambda}$ stands for the reduction of the full oneloop determinant to its Einstein-Hilbert part with a curvature term and a cosmological constant term. The respective coefficients can be absorbed in a redefinition of the Newton constant and cosmological constant in $S_{\mathrm{EH}}[g]$. (For more details, see 202. Hence, $\mathbf{1 0 1}$ is equivalent to the Einstein-Hilbert truncation of the pure gravity system. The latter shows the Reuter fixed point and so should the minimally coupled system in this truncation.

The above result for minimally coupled systems has the direct consequence that the Einstein-Hilbert truncation to matter-gravity systems should also exhibit the Reuter fixed point for UV-complete matter systems, as the pure gravity system does. We add that this does not exclude the emergence of further fixed points in some $N_{\text {mat }}$ regime.

This concludes our discussion of the fixed-point structure and stability properties of gravity-matter systems, its truncation dependence, and reliability requirements for truncations. The discussion enables us to formulate relevant properties that have to be considered for a conclusive stability analysis of matter-gravity systems:

(1) The fixed-point analysis necessarily has to involve all (possibly) relevant operators of the theory under investigation, that is, 99 in many-flavor QCD and 100 in gravity-matter systems.

(2) A fixed-point analysis within a given truncation is only fully reliable if it also reproduces the fixed-points of the pure gravity system in the same truncation excluding the marginal operators.

We now discuss the results in gravity-matter systems given the properties i) and ii): In [201], the first full fluctuation computation for minimally-coupled systems was put forward. On the pure gravity side, the flows of the fluctuation graviton twoand three-point function were included. Importantly, a stabilizing mechanism for the fermionic contribution was found for general regulators: the graviton mass parameter is approaching its pole $\mu \rightarrow-1$ and thus enhances the graviton contribution, in short: gravity rules. This is required from the discussion above. Technically, this simply means that the fermion contribution in this setup changes the parameters of the two- and three-point function within the stability regime of the phase diagram of pure gravity in the Einstein-Hilbert truncation. This stabilizing mechanism was also found in an extension of the truncation [204], making the fermion-gravity system a showcase of the mechanism described above. In particular, with the existence of the Reuter fixed point for the minimally coupled system in the absence of marginal operators in the pure gravity subsystem, the flow equations of the fermion-gravity system satisfy the requirement ii). Consequently, a conclusive stability analysis of general fermion-gravity systems can be performed but requires the inclusion of the marginal curvature-squared operators.

In the same truncation applied to minimally coupled scalar-gravity systems, it was found within the fluctuation approach in 93, 201 that the graviton anomalous dimension $\eta_{h}$ grows with the number of scalars $N_{s}$ and finally exceeds the value two beyond a critical flavor number $N_{s, \text { stab }}$ : for $N_{s}>N_{s, \text { stab }} \approx 20$. For $\eta_{h}>2$, the overall cutoff scaling of the graviton regulator goes with negative powers of the cutoff scales and effectively the-physical-cutoff decreases. For these large anomalous dimensions, we leave the reliability regime of the approximation. In short, the reliability bound on the truncation makes it impossible to see the stability of the system in this minimally coupled approximation. From the viewpoint of the pure gravity system, this simply means that the scalar contribution in this setup eventually moves the parameters of the two- and three-point function outside the stability regime of the phase diagram of pure gravity in the Einstein-Hilbert truncation. Consequently, the setup cannot be used for stability investigations in scalar-gravity systems. In 97, it was suggested that an expansion about an on-shell background can lift this tension. In summary, at present, there is no conclusive stability analysis for scalar-gravity systems.

Applying the same truncation to minimally coupled gauge-gravity systems, it has been shown in 202 that depending on the regulator, the minimally coupled systems either behave similarly to the fermionic or the scalar system. This suggests that the truncation has to be improved. In summary, a stability analysis of gauge-gravity systems can be performed, but the results have to be taken with a grain of salt. A fully conclusive stability analysis for gauge-gravity systems requires an improvement of the truncations used so far in the literature.

In Figure 9, we display the state-of-the-art dependence of the fixed-point values on the number of scalar field $N_{s}$ [93], fermion field $N_{f}$ [204], and gauge fields $N_{v}$ [202]. The truncations include the flow of the momentum-dependent graviton two- and threepoint functions as well as the respective graviton-matter vertex. In the scalar case, the Newton couplings are diverging at $N_{s} \approx 52$. This is an artifact of the truncation, as described in the previous paragraphs and 202. The fermion direction is stable for all $N_{f}$ : the graviton mass parameter approaches its pole $\mu \rightarrow-1$ and the enhanced graviton contribution counterbalances the matter contribution. In the gauge case, the fixed point is disappearing 


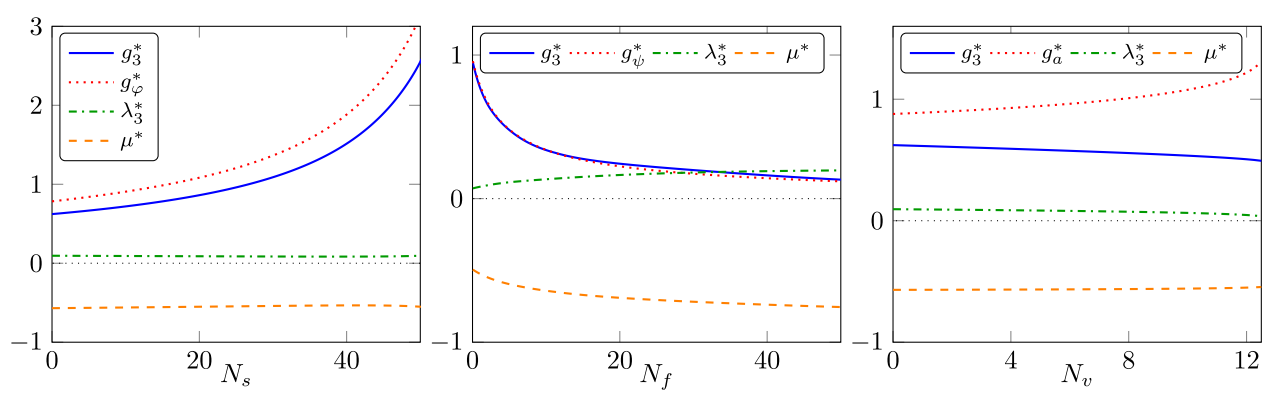

FIGURE 9|Fixed-point values of the fluctuation couplings as a function of the number of scalar (left), fermion (middle), and gauge fields (right). All truncations include the graviton two- and three-point function as well as the respective graviton-matter vertex. In the scalar case, the Newton couplings, $g_{3}$ and $g_{\varphi}$, are diverging at $N_{s} \approx 52$. The fermionic case is stable for all $N_{f}$. In the gauge field case, the fixed point is disappearing in the complex plane at $N_{v} \approx 13$. It was explained in 202 that the vanishing of the fixed point is an artifact of the truncation and how it can be lifted in the gauge-field case. In 97 , it was suggested that an expansion about a background that is a solution to the quantum EoM might remove the divergence in the scalar case. The result are taken from 93 (scalar), 204 , (fermion), and 202 (gauge).

in the complex plane for $N_{v} \approx 13$. In 202, it was demonstrated that all numbers of gauge fields can be accessed with a different regulator, as discussed in the last paragraph.

Finally, we speculate on the stability properties of general gravity-matter systems based on the results obtained so far. To that end, we assume that there is a setup such that general minimally coupled gravity-matter systems in the Einstein-Hilbert truncation show UV stability with a Reuter fixed point similar to the one seen in the fermion-gravity system. This property allows for a consistent truncation as it satisfies ii). Now, we include tensor structures from curvaturesquared terms, $R^{2}, R_{\mu \nu}^{2}$, and $R_{\mu \nu \rho \sigma}^{2}$. It is convenient to parameterize this complete set of tensor structures in terms of the Ricci squared, the Weyl tensor squared, and the topological Gauß-Bonnet term,

$$
\int \mathrm{d}^{4} x \sqrt{g}\left(\frac{1}{g_{R^{2}}} R^{2}+\frac{1}{g_{C^{2}}} C_{\mu \nu \rho \sigma}^{2}+\frac{1}{g_{E}} E\right),
$$

with the dimensionless couplings $g_{R^{2}}, g_{C^{2}}$, and $g_{E}$. The Gauß-Bonnet density $E$ is defined in $\mathbf{8 1}$, and the Weyl tensor squared is in four dimensions given by

$$
C_{\mu \nu \rho \sigma}^{2}=R_{\mu \nu \rho \sigma}^{2}-2 R_{\mu \nu}^{2}+\frac{1}{3} R^{2}
$$

We concentrate on the Reuter fixed point with the assumption that it is dominated by the Einstein-Hilbert couplings in contradistinction to the perturbative $R^{2}$ fixed point. In 184, it has been observed that $R_{\mu \nu}^{2}$ contributions and hence $C^{2}$ contributions generated by the Einstein-Hilbert tensor structures at the Reuter fixed point are small. They are subleading in comparison to the $R^{2}$ tensor structure. This has been the topic of Section $\mathbf{8 . 3}$ (see Figure 8 and the respective discussion). With the assumption of the dominance of the Einstein-Hilbert couplings, it implies $1 / g_{C^{2}}^{*} \approx 0$ and indicates the irrelevance of this operator at the fixed point.

Moreover, from the quartic term $c_{4} p^{4}$ with $c_{4} \approx-0.24$ in the running of the momentum-dependent coupling of the four-point function displayed in Figure 8, we deduce that its contribution
$c_{\mathrm{EH}} g_{R^{2}}^{2}$ to the $\beta$-function $\beta_{g_{R^{2}}}=\partial_{t} g_{R^{2}}$ of the $R^{2}$ tensor structure is positive. (For a detailed discussion, see 184.) In turn, it is wellknown that the $R^{2}$ coupling itself leads to a negative contribution $-c_{R^{2}}\left(g_{R^{2}}\right)$, which is one-loop universal. We emphasize that both coefficients depend on the full fluctuation propagator. In combination, this leads us to a $\beta$-function

$$
\partial_{t} g_{R^{2}}=\beta_{g_{R^{2}}} \simeq c_{\mathrm{EH}} g_{R^{2}}^{2}-c_{R^{2}}\left(g_{R^{2}}\right) .
$$

Switching off the Einstein-Hilbert contribution leads us to the standard Gaußian fixed point for $R^{2}$ gravity. In turn, at the Reuter fixed point, we assume a small fixed-point value for $1 / g_{R^{2}}$ that may also trigger a small, but nonvanishing fixed-point value for $1 / g_{C^{2}}$. Combining these estimates for $g_{R^{2}}$ and $g_{C^{2}}$, we arrive at

$$
\frac{1}{g_{R^{2}}^{*}}, \frac{1}{g_{C^{2}}^{*}} \approx 0, \quad \text { and } \quad \frac{g_{C^{2}}^{*}}{g_{R^{2}}^{*}} \approx 0,
$$

in pure gravity. We add that the relevance analysis in 184 suggests that the $g_{R^{2}}$ coupling, while being small, is UV-relevant at the Reuter fixed point. This finding is corroborated by respective ones in the background approximation. (For higher derivative gravity work in the background field approximation, see, e.g., 49, 51, 54, 56, 58, 108-110, 172-174, 176, 183, 186, 189, 219, 265-269].)

We now proceed to the $R^{2}$ and $C^{2}$ contributions from matter fluctuations. Being short of a full fluctuation computation of these terms, we utilize the Nielsen identities in the presence of the cutoff (see 54 and 44 in Sections 5.2 and 6). The identity 44 comprises the difference between background-metric and fluctuation field derivatives, while the Nielsen identities $\mathbf{5 4}$ also take into account the difference introduced by the gauge fixing sector. For the present speculative analysis, it suffices to discuss 44. For example, we find for the $R^{2}$ contribution,

$$
\left.\left[\frac{\delta \Gamma_{k}}{\delta \bar{g}}-\frac{\delta \Gamma_{k}}{\delta h}\right]_{R^{2}} \simeq \operatorname{Tr} \frac{\delta \sqrt{\bar{g}} R_{k}}{\sqrt{\bar{g}} \delta \bar{g}_{\mu \nu}} G_{k}\right|_{R^{2}}=\Delta g_{R^{2}}(\vec{g}) \int \mathrm{d}^{4} x \sqrt{\bar{g}} R^{2},
$$

The right-hand side has a form similar to the flow equation itself and is UV- and IR-finite. Accordingly, $\Delta g_{R^{2}}(\vec{g})$ is a 
dimensionless constant that depends on all couplings taken into account in the computation, summarized as vector $\vec{g}$. This includes the $R^{2}$ and $C^{2}$ couplings $g_{R^{2}}$ and $g_{C^{2}}$ themselves (or rather avatars thereof), as well as avatars of the dimensionless Newton coupling and the dimensionless cosmological constant (see Section 8.1). The scale derivative of $\mathbf{1 0 6}$ vanishes on a fixed point,

$$
\partial_{t}\left[\frac{\delta \Gamma_{k}}{\delta \bar{g}}-\frac{\delta \Gamma_{k}}{\delta h}\right]_{R^{2}} \simeq \frac{\partial \Delta g_{R^{2}}\left(\vec{g}^{*}\right)}{\partial g_{i}} \beta_{i}\left(\vec{g}^{*}\right)=0,
$$

where we have used that the dimensionless coefficient $\Delta g_{R^{2}}\left(\vec{g}^{*}\right)$ cannot have an explicit $k$-dependence. Hence, at a fixed point, this result allows us to identify the matter contribution of the flow for $R^{2}$ tensor structures of fluctuation field vertices with that of the background field $R^{2}$ term. The same reasoning also applies to the $C^{2}$ term. In summary, the above arguments imply that the matter contributions to the curvature-squared couplings should be independent of the background-metric dependence of the regulator, as well as of the shape of the regulator. Moreover, since the ghost contribution to the curvature-squared couplings also does not depend on other scales than the cutoff scale, it should also be regulator-independent. The validity of these general statements can be checked explicitly with the results of 58,183 . There, different types of regulators have been investigated in $f(r)$ gravity: all couplings, except the $R^{2}$-coupling, depend on the Laplacian used in the regulators. The results also confirm a regulator dependence of the graviton contributions, triggered by the Nielsen identities. As discussed above, this suggests that the pure gravity contributions to the flow should rather be computed within the fluctuation approach.

The above considerations allow us to discuss the generic structure of gravity matter flows within the fluctuation approach,

$$
\begin{aligned}
& \beta_{g_{R^{2}}}=\left.\beta_{g_{R^{2}}}\right|_{\text {grav }}-c_{R^{2}} N_{R^{2}} g_{R^{2}}^{2}, \\
& \beta_{g_{C^{2}}}=\left.\beta_{g_{C^{2}}}\right|_{\text {grav }}-c_{C^{2}} N_{C^{2}} g_{C^{2}}^{2},
\end{aligned}
$$

where $c_{R^{2} / C^{2}}$ are positive coefficients and $N_{R^{2} / C^{2}}$ are weighted sums (positive weights) of the numbers of scalars, vectors, and fermions. All matter contributions have the same sign, which is the same as that of the gravity-ghost, which is computationally similar. (For explicit computations in the background field approximation, see, e.g., 58, 183, 186, 219.)

The quantitative evaluation of $\mathbf{1 0 8}$ depends on the full fluctuation flows in pure gravity including flow contributions from curvature-squared invariants. Here, we concentrate on the structure of the $\beta$-function of the $R^{2}$ coupling, $\beta_{g_{R^{2}}}$. The matter contributions are subtracted from the positive Einstein-Hilbert gravity contribution (see $c_{\mathrm{EH}}$ in 104). For a critical number of matter fields, the complete contribution vanishes, and we are left with a system that resembles the pure gravity curvature-squared system. This mechanism is very similar, leading to the Caswell-Banks-Zaks fixed point in QCD discussed before. Note that in contradistinction to the minimally coupled system, the matter contribution cannot be absorbed in the pure gravity contributions, as they are related to
$R^{2} \log \left(1+R / k^{2}\right)$ terms. This is visible in the limit of large curvatures (see, e.g., 183). This qualitative analysis has to be sustained with a quantitative computation based on pure gravity flows including higher curvature terms. Such a computation requires improved truncations with the properties i) and ii).

We close this chapter with a brief overview of investigations of gravity-matter systems within the background field or hybrid approximations. In 219, gravity-matter systems in the minimally coupled approximation were investigated in a hybrid approach: while most contributions to the flow have been computed in the background field approximation, the matter parts of the anomalous dimensions have been computed in a fluctuation approach setup. Within this approximation, destabilizing effects for scalars and fermions and stabilizing effects for gauge fields were found. The destabilizing result for fermions in 219 is an artifact of the background field approximation, as discussed in destabilizing result for Section 8.5: the backgroundmetric dependence of the regulator influences the (de)stabilizing property of minimally coupled fermions. However, this does not imply that the background field approximation breaks down for all gravity couplings. The results of 201, 202 showed that in particular, the most UV-relevant operators have to be taken from a fluctuation computation, that is, most importantly the graviton mass parameter $\mu$. In turn, the background and the fluctuation Newton coupling behave rather similar under the influence of minimally coupled matter fields. The sign of leading-order contribution agrees: the scalar and fermionic contribution to the beta function of the Newton coupling at $\mathcal{O}\left(g^{2}\right)$ is positive, while the gauge contribution is negative.

In summary, the investigations of gravity-matter systems within the fluctuation approach open a systematic path toward reliable stability investigations of fully coupled matter systems as well as that of phenomenological consequences for high energy physics. Still, fully reliable results require a systematic and qualitative improvement of the current truncations. This is the subject of current work in the community.

\subsection{Effective Universality}

In the vertex expansion $\mathbf{6 8}$, we have introduced the couplings $g_{n}$ for each graviton $n$-point function as the running couplings of the Ricci scalar tensor structure $(\sqrt{g} R)^{(n)}$ (see Section 8.3). In a diffeomorphism-invariant approach, these couplings would agree. In turn, in the present gauge-fixed approach, these are different avatars of the Newton coupling. While not being identical, $g_{i} \neq g_{j}$, they are related by nontrivial mSTIs (65).

This is similar in non-abelian gauge theories, where different avatars of the running strong coupling $\alpha_{s}=g^{2} /(4 \pi)$ can be derived from different correlation functions, both from pure glue vertices as well as glue-matter vertices. (For a detailed discussion, see $167,168,170$ and the recent review 27.) The $\beta$-functions of all the avatars of the strong coupling are two-loop universal in mass-independent renormalization schemes, or may also define an RG scheme with the requirement that $\beta$-functions agree to all orders. However, the standard fRG renormalization scheme is mass-dependent, so even two-loop universality is not guaranteed. More importantly, identical $\beta$-functions do not necessarily lead to an identical momentum dependence. 
Indeed, in non-abelian gauge theories, the momentum dependence of different avatars of the running strong coupling differs already at the universal two-loop order, which can be also shown from the STIs. Additionally, in the strongly correlated IR regime of a non-abelian gauge theory, the fRG $\beta$-functions and the momentum dependence of the running couplings differ significantly. Some of them, that is, the three-gluon coupling, even switch sign, while others, that is, the ghost-gluon and fourgluon coupling, stay positive [168].

In gravity, the situation is even more intricate. To begin with, the Newton coupling is dimensionful, and hence, the $\beta$-functions of the avatars of the Newton coupling are not universal, leaving aside an identical momentum dependence. Additionally, as already mentioned in the context of non-abelian gauge theories, the standard fRG renormalization schemes are typically mass-dependent, which adds to the differences, as do truncations.

Effective universality is the concept that in particular at the fixed point, where gravity is in a scaling regime, and the quantum theory is dominated by the diffeomorphism invariance of the underlying theory. If this scenario applies, the $\beta$-functions and the momentum dependence of different avatars of the Newton coupling should agree or are rather be close to each other on the asymptotically safe UV fixed point. This concept would apply to all couplings, and in particular, the $\lambda_{n}$ can be understood as avatars of the cosmological constant. Additionally to the Newton couplings from the Ricci scalar tensor structure, we have further avatars of the Newton coupling stemming from the gravity-matter correlation functions.

Given the presence of truncations in explicit computations, the impact of nontrivial $\mathrm{mSTI}$ and the nonperturbative nature of the UV fixed point, it is left to define a measure for effective universality. In 93, 203, it was quantified how these avatars differ at the UV fixed point using the measure

$$
\varepsilon_{i j}\left(g, \mu, \lambda_{3}\right)=\left|\frac{\Delta \beta_{g_{i}}-\Delta \beta_{g_{j}}}{\Delta \beta_{g_{i}}+\Delta \beta_{g_{j}}}\right|_{g_{i}=g_{j}=g},
$$

where $\Delta \beta_{g_{i}}$ is the anomalous part of the $\beta$-function $\beta_{g_{i}}$ obtained by subtracting the canonical running

$$
\Delta \beta_{g_{i}}=\beta_{g_{i}}-2 g_{i}
$$

In 203, five avatars of the Newton coupling were included stemming from the three-point functions, $\Gamma_{k}^{(h h h)}, \Gamma_{k}^{(c c h)}, \Gamma_{k}^{(\varphi \varphi h)}$, $\Gamma_{k}^{(\psi \psi h)}$, and $\Gamma_{k}^{(A A h)}$. Thus, the set of $g_{i}$ is given by $i \in\{h, c, \varphi, \psi, A\}$, where $g_{h}=g_{3}$ in the previous notation. In 109, the $\beta$-functions are identical for $\varepsilon_{i j}=0$, and we have full universality. A small value of $\varepsilon_{i j}$ indicates almost identical $\beta$-functions and thus "effective universality." In 203, these small values were estimated to be $\varepsilon_{i j}<0.2$. This estimate is based on a systematic error estimate of the used truncations and the impact of the mSTIs. In turn, a larger value of $\varepsilon_{i j}$ shows that universality is strongly broken and that the mSTIs are highly nontrivial.

The universality measures $\varepsilon_{i j}$ are functions of all couplings, and we display them in Figure 10 for $g_{i}=g_{h}^{*}$ as functions of $\mu$ and $\lambda_{3}$. Remarkably, the UV fixed point lies in the green area, which signals $\varepsilon<0.2$, and thus, effective universality holds. As discussed above, this statement is nontrivial since the mSTIs can introduce large differences between the avatars, in particular, if the fixed point is highly nonperturbative. In turn, this result gives a strong hint that the UV fixed point is in the semiperturbative region. Interestingly, a semiperturbative behavior was also found in large-order Ricci scalar expansions of the effective action in the background field approximation [108-110, 172, 173]. There it was found that the critical exponents of the highorder curvature invariants are close to their canonical values.

We emphasize that the observed effective universality is a highly nontrivial result. If it can be sustained in further analyses, it is presumably dynamical. This conjecture is supported by the following observation: for a marginal universal coupling, one may simply compute one avatar of the coupling and identify the other avatars with the computed one. In turn, in a theory like gravity, where the effective universality is potentially generated dynamically, this may only work in specific RG schemes. One may even define a natural RG scheme by $\epsilon_{i j} \equiv 0$. This entails that in other RG schemes, only a subset of the couplings will have the natural $\beta$-functions. Note that the latter property is additionally triggered by the inherent truncations of explicit computations.

In any case, within a given RG scheme, some of the $\beta$-functions may satisfy $\epsilon_{i j} \equiv 0$, while others may not. The identification of all avatars of the given coupling with a specific one will only work if the

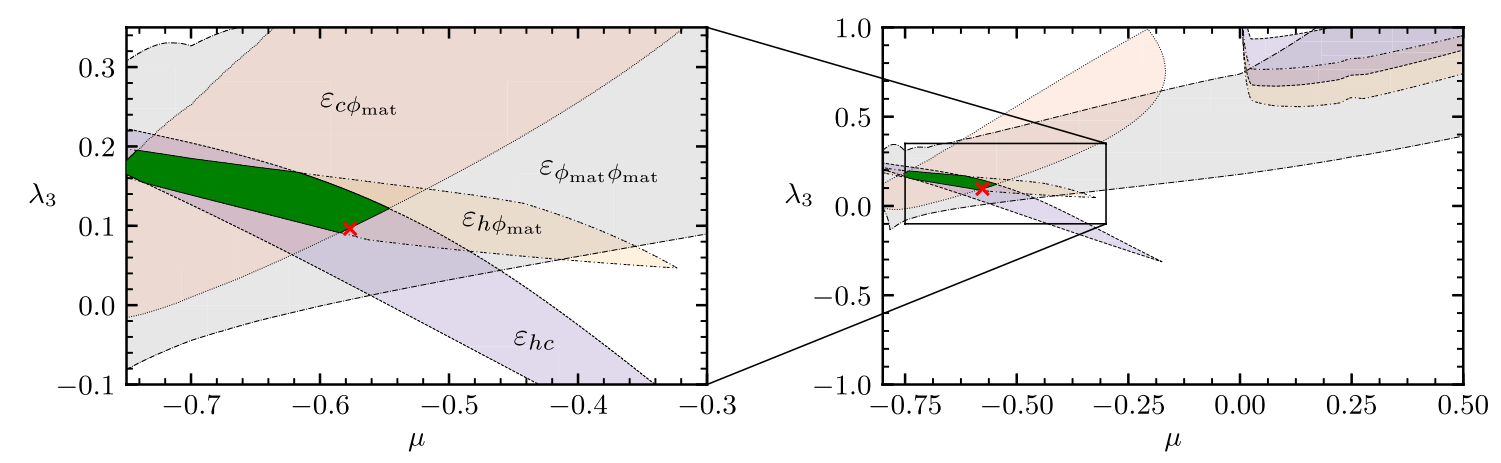

FIGURE 10 | Effective universality of the different avatars of the Newton coupling as a function of $\mu$ and $\lambda_{3}$. The regions of effective universality are defined with $\varepsilon_{i j}<0.2$ according to 109 . The red cross indicates the UV fixed point, which lies in the region of effective universality. The figure is taken from 203. 
latter coupling is chosen from the natural subset. Such an identification is an implicit way of enforcing the natural RG scheme. In turn, if all couplings are identified with an avatar which is not in the natural subset, the system may be corrupted. This can even lead to a loss of the fixed point.

In gravity-matter systems, we indeed observe, in given truncations, such a behavior: if all avatars of the Newton coupling are identified with the three-graviton coupling $g_{h}$, that is, $g_{i}=g_{h}$, the results are close to the full ones with multiple avatars of the Newton coupling. In turn, identifying all avatars of the Newton coupling with a gravity-matter avatar fails. In summary, this hints at a surprisingly complicated interaction structure in gravity-matter systems. Its origin is yet to be understood and may give us further valuable insights into the dynamics of these systems. In short, these investigations of effective universality indicate a close perturbativeness of the UV fixed-point regime of asymptotically safe gravity.

\section{SUMMARY AND OUTLOOK}

In this contribution, we have reviewed the state of the art of the fluctuation approach to quantum gravity. This approach is based upon the computation of the correlation functions of the dynamical graviton fluctuation field $h_{\mu \nu}$ within a systematic vertex expansion. This can be done within general parameterizations of the full metric, but most results have been achieved in the linear split, $g_{\mu \nu}=\bar{g}_{\mu \nu}+h_{\mu \nu}$. While the correlation functions of the fluctuation field are not observables by themselves and carry a gauge dependence, the computation of observables in quantum gravity requires the knowledge of the fluctuation correlation functions, and they indeed encode the dynamics of quantum gravity.

By now, the fluctuation approach has matured (see the overview of the results in Section 8). We see signs of apparent

\section{REFERENCES}

1. Weinberg S. General relativity: an Einstein centenary survey. SW Hawking W Israel editors. Cambridge, England: Cambridge University Press (1980)

2. Wetterich C. Exact evolution equation for the effective potential. Phys Lett $B$ (1993) 301 (1):90-4. doi:10.1016/0370-2693(93)90726-x

3. Reuter M. Nonperturbative evolution equation for quantum gravity. Phys Rev $D$ (1998) 57:971-85. doi:10.1103/physrevd.57.971

4. Souma W. Non-trivial ultraviolet fixed point in quantum gravity. Prog Theor Phys (1999) 102:181-95. doi:10.1143/PTP.102.181

5. Percacci R. An introduction to covariant quantum gravity and asymptotic safety. In: 100 years of general relativity, Vol. 3. Singapore: World Scientific (2017). doi:10.1142/10369

6. Reuter M, Saueressig F. Quantum gravity and the functional renormalization group. Cambridge, England: Cambridge University Press (2019). doi:10.1017/ 9781316227596

7. Niedermaier $M$, Reuter $M$. The asymptotic safety scenario in quantum gravity. Living Rev Relat (2006) 9:5. doi:10.12942/lrr-2006-5

8. Litim DF. Renormalization group and the Planck scale. Phil Trans $R$ Soc A (2011) 369(1946):2759. doi:10.1098/rsta.2011.0103

9. Reuter M, Saueressig F. Quantum Einstein gravity. New J Phys (2012) 14: 055022. doi:10.1088/1367-2630/14/5/055022 convergence of the results in pure gravity. Moreover, by now, we can reliably evaluate the stability of general gravity-matter systems. In combination, the fluctuation approach now allows for reliable physics predictions for the UV regime of asymptotically safe gravity including its unitarity. The approach also allows for reliable physics predictions for the "IR" particle physics within the asymptotically safe standard model.

\section{AUTHOR CONTRIBUTIONS}

All authors listed have made a substantial, direct, and intellectual contribution to the work and approved it for publication.

\section{FUNDING}

This work is supported by the DFG, Project-ID 273811115, SFB 1225 (ISOQUANT), as well as by the DFG under Germany's Excellence Strategy EXC - 2181/1-390900948 (the Heidelberg Excellence Cluster STRUCTURES), by the Danish National Research Foundation under Grant No. DNRF:90, and by the Science and Technology Research Council (STFC) under the Consolidated Grant ST/T00102X/1.

\section{ACKNOWLEDGMENTS}

We thank A. Bonanno, B. Bürger, N. Christiansen, T. Denz, A. Eichhorn, K. Falls, H. Gies, A. Held, B. Knorr, P. Labus, S. Lippoldt, J. Meibohm, D. Litim, A. Pereira, A. Rodigast, B.-J. Schaefer, M. Schiffer, F. Saueressig, J. Smirnov, M. Yamada, and C. Wetterich for discussions and work on the subjects reported on.

10. Ashtekar A, Reuter M, Rovelli C. From general relativity to quantum gravity. Gen Relativity Quantum Cosmol (2014) Preprint arXiv:1408.4336.

11. Eichhorn A. Unimodular quantum gravity and the cosmological constant. In: Proceedings of the Black holes, gravitational waves and spacetime singularities; December 2017; Rome, Italy: Springer. Vol. 8, p. 1364-1379.

12. Bonanno A, Saueressig F. Asymptotically safe cosmology - a status report. Compt Rendus Phys (2017) 18:254. doi:10.1016/j.crhy.2017.02.002

13. Eichhorn A. An asymptotically safe guide to quantum gravity and matter. Front Astron Space Sci (2019) 5:47. doi:10.3389/fspas.2018.00047

14. Pereira AD. Progress and visions in quantum theory in view of gravity: bridging foundations of physics and mathematics. London: Springer Nature (2019)

15. Reichert M Modave 2019, PoS 005 (2020)

16. Bonanno A, Eichhorn A, Gies H, Pawlowski JM, Percacci R, Reuter M, et al. Critical reflections on asymptotically safe gravity. Front Physiol (2020) 8:269. doi:10.3389/fphy.2020.00269

17. Donoghue JF. A critique of the asymptotic safety program. Front Phys (2020) 8: 56. doi:10.3389/fphy.2020.00056

18. Berges J, Tetradis N, Wetterich C, Non-perturbative renormalization flow in quantum field theory and statistical physics. Phys Rep (2002) 363:223. doi:10. 1016/s0370-1573(01)00098-9

19. Aoki K. Introduction to the nonperturbative renormalization group and its recent applications. Int J Mod Phys E (2000) 14:1249-1326. doi:10.1142/ s0217979200000923 
20. Polonyi J. Lectures on the functional renormalization group method. Cent Eur J Phys (2003) 1:1-71. doi:10.2478/BF02475552

21. Pawlowski JM, Aspects of the functional renormalisation group. Ann Phys (2007) 322:2831. doi:10.1016/j.aop.2007.01.007

22. Gies $H$. Introduction to the functional RG and applications to gauge theories. Lect Notes Phys (2012) 852:287. doi:10.1007/978-3-642-27320-9_6

23. Delamotte B. An introduction to the nonperturbative renormalization group. In: Renormalization group and effective field theory approaches to many-body systems, Lecture Notes in Physics. Berlin: Springer, 49-132. doi:10.1007/978-3642-27320-9_2

24. Kopietz P, Bartosch L, Schutz F. Introduction to the functional renormalization group. Lect. Notes Phys (2010) doi:10.1007/978-3-64205094-7

25. Rosten OJ, (2010) On the renormalization of theories of a scalar chiral superfield. JHEP 4, doi:10.1007/JHEP03(2010)004

26. Braun J. J Phys (2012) G39:033001.

27. Dupuis N, Canet L, Eichhorn A, Metzner W, Pawlowski J, Tissier M, et al. (2020)

28. 't Hooft G, Veltman MJG. One-loop divergencies in the theory of gravitation. Ann Inst H Poincare Phys Theor (1974) A20:69-94.

29. Goroff MH, Sagnotti A, Sagnotti A. Quantum gravity at two loops Phys Lett B (1985) 160:81. doi:10.1016/0370-2693(85)91470-4

30. Goroff MH, Sagnotti A. The ultraviolet behavior of Einstein gravity. Nucl Phys $B$ (1986) 266:709. doi:10.1016/0550-3213(86)90193-8

31. van de Ven AEM. Two-loop quantum gravity. Nucl Phys B (1992) 378:309. doi:10.1016/0550-3213(92)90011-y

32. Stelle KS. Renormalization of higher-derivative quantum gravity. Phys Rev D (1977) 16:953. doi:10.1103/physrevd.16.953

33. Stelle KS. Classical gravity with higher derivatives. Gen Relat Gravit (1978) 9: 353. doi:10.1007/bf00760427

34. Antoniadis I, Tomboulis ET. Gauge invariance and unitarity in higherderivative quantum gravity. Phys Rev D (1986) 33:2756. doi:10.1103/ physrevd.33.2756

35. Houthoff WB, Kurov A, Saueressig F. Impact of topology in foliated quantum Einstein gravity. Eur Phys J (2017) C77:491. doi:10.1140/epjc/s10052-0175046-8

36. Ambjorn J, Jurkiewicz J. Phys Lett (1992) B278:42.

37. Bilke S, Burda Z, Petersson B. Topology in $4 \mathrm{D}$ simplicial quantum gravity. Phys Lett B (1997) 395:4. doi:10.1016/s0370-2693(97)00047-6

38. Ambjorn J, Loll R. Non-perturbative Lorentzian quantum gravity, causality and topology change. Nucl Phys B (1998) 536:407. doi:10.1016/s0550-3213(98) 00692-0

39. Ambjorn J, Jurkiewicz J, Loll R. Emergence of a $4 \mathrm{D}$ world from causal quantum gravity. Phys Rev Lett (2004) 93:131301. doi:10.1103/physrevlett.93.131301

40. Ambjorn J, Jurkiewicz J, Loll R. Reconstructing the universe. Phys Rev (2005) D72:064014. doi:10.1103/physrevd.72.064014

41. Laiho J, Coumbe D. Evidence for asymptotic safety from lattice quantum gravity. Phys Rev Lett (2011) 107:161301. doi:10.1103/physrevlett.107.161301

42. Ambjørn J, Drogosz Z, Gizbert-Studnicki J, Görlich A, Jurkiewicz J, Nemeth D. Impact of topology in causal dynamical triangulations quantum gravity. Phys Rev D (2016) 94:044010. doi:10.1103/physrevd.94.044010

43. Kawai H, Kitazawa Y, Ninomiya M. Scaling exponents in quantum gravity near two dimensions. Nucl Phys B (1993) 393:280. doi:10.1016/0550-3213(93) 90246-1

44. Nink A. Field parametrization dependence in asymptotically safe quantum gravity. Phys Rev D (2015) 91:044030. doi:10.1103/PhysRevD.91.044030

45. Falls K. Renormalisation of Newton's constant. Phys Rev D (2015) 92:124057. doi:10.1103/PhysRevD.92.124057

46. Demmel M, Nink A. Connections and geodesics in the space of metrics. Phys Rev D (2015) 92:104013. doi:10.1103/PhysRevD.92.104013

47. Percacci R, Vacca GP. Search of scaling solutions in scalar-tensor gravity. Eur Phys J C (2015) 75:188.

48. Gies H, Knorr B, Lippoldt S. Generalized parametrization dependence in quantum gravity. Phys Rev D (2015) 92:084020. doi:10.1103/PhysRevD.92. 084020

49. Ohta N, Percacci R, Vacca GP. Flow equation for $f(R)$ gravity and some of its exact solutions. Phys Rev D (2015) 92:061501. doi:10.1103/PhysRevD.92. 061501
50. Labus P, Percacci R, Vacca GP. Asymptotic safety in O (N) scalar models coupled to gravity. Phys Lett B (2016) 753:274. doi:10.1016/j.physletb.2015. 12.022

51. Ohta N, Percacci R, Vacca GP. Eur Phys J C (2016) C76:46.

52. Ohta N, Percacci R. Classical Quant Grav (2016) 33:035001. doi:10.1088/02649381/33/3/035001

53. Donà P, Eichhorn A, Labus P, Percacci R. Phys. Rev.Phys. Rev (2016) D93: 129904.

54. Falls K, Ohta N. Phys Rev (2016) D94:084005.

55. Ohta N, Percacci R, Pereira AD. JHEP (2016) 06:115.

56. Ohta N, Percacci R, Pereira AD. Eur Phys J (2017) C77:611.

57. Ohta N. PTEP (2017):033E02.

58. Alkofer N, Saueressig F. Asymptotically safe $\mathrm{f}(\mathrm{R})$-gravity coupled to matter I: the polynomial case. Ann Phys (2018) 396:173. doi:10.1016/j.aop.2018. 07.017

59. De Brito GP, Ohta N, Pereira AD, Tomaz AA, Yamada M. Phys Rev (2018) D98:026027.

60. Eichhorn A. On unimodular quantum gravity. Classical Quant Grav (2013) 30: 115016. doi:10.1088/0264-9381/30/11/115016

61. Eichhorn A. JHEP (2015) 04:096

62. Benedetti D. Gen Relat Gravit (2016) 48:68. doi:10.1007/s10714-016-2060-3 63. de León Ardón R, Ohta N, Percacci R. Phys Rev (2018) D97:026007.

64. Percacci R. Unimodular quantum gravity and the cosmological constant. Found Phys (2018) 48:1364. doi:10.1007/s10701-018-0189-5

65. De Brito GP, Eichhorn A, Pereira AD. JHEP (2019a) 09:100.

66. de Brito GP, Pereira AD, (2020)

67. DeWitt BS. Oxford conference on quantum gravity (1980). p. 449-87.

68. Fradkin ES, Tseytlin AA. On the new definition of off-shell effective action. Nucl Phys B (1984) 234:509. doi:10.1016/0550-3213(84)90075-0

69. Vilkovisky GA. The unique effective action in quantum field theory. Nucl Phys $B$ (1984) 234:125. doi:10.1016/0550-3213(84)90228-1

70. DeWitt BS The global approach to quantum field theory, 1 (2003).

71. Branchina V, Meissner KA, Veneziano G. The price of an exact, gaugeinvariant RG-flow equation. Phys Lett B (2003) 574:319. doi:10.1016/j. physletb.2003.09.020

72. Pawlowski JM, (2003)

73. Donkin I, Pawlowski JM, (2012)

74. Demmel M, Saueressig F, Zanusso O. RG flows of quantum Einstein gravity in the linear-geometric approximation. Ann Phys (2015) 359:141. doi:10.1016/j. aop. 2015.04 .018

75. Falls K, (2020), 11409

76. Pawlowski JM, Litim DF, Nedelko S, von Smekal L. Phys Rev Lett (2004) 93: 152002. doi:10.1103/physrevlett.93.152002

77. Pawlowski JM, Scherer MM, Schmidt R, Wetzel SJ, Physics and the choice of regulators in functional renormalisation group flows. Ann Phys (2017) 384: 165. doi:10.1016/j.aop.2017.06.017

78. Ellwanger U. Proceedings, Workshop on Quantum field theoretical aspects of high energy physics: bad Frankenhausen, Germany, September 20-24, 1993. Z Phys (1994) C62:503.

79. Morris TR, The exact renormalization group and approximate solutions. Int J Mod Phys (1994) 09:2411. doi:10.1142/s0217751x94000972

80. Reuter M, Wetterich C. Effective average action for gauge theories and exact evolution equations. Nucl Phys B (1994) 417:181. doi:10.1016/0550-3213(94) 90543-6

81. Litim DF, Pawlowski JM. Phys Lett (2002) B546:279.

82. Litim DF, Pawlowski JM. Renormalisation group flows for gauge theories in axial gauges. J High Energy Phys (2002) 2002:049. doi:10.1088/1126-6708/ 2002/09/049

83. Folkerts S, Litim DF, Pawlowski JM, Asymptotic freedom of Yang-Mills theory with gravity. Phys Lett B (2012) 709:234. doi:10.1016/j.physletb.2012.02.002

84. Braun J, Leonhardt M, Pawlowski JM. SciPost Phys (2019) 6:056. doi:10.21468/ scipostphys.6.5.056

85. Pawlowski JM. On Wilsonian flows in gauge theories. Int J Mod Phys (2001) 16: 2105. doi:10.1142/s0217751x01004785

86. Pawlowski JM. Acta Phys Slov (2002) 52:475.

87. Morris TR. JHEP (2016) 11:160.

88. Percacci R, Vacca GP. Eur Phys J (2017) C77:52.

89. Labus P, Morris TR, Slade ZH. Phys Rev (2016) D94:024007. 
90. Nieto CM, Percacci R, Skrinjar V. Phys Rev (2017) D96:106019.

91. Bridle IH, Dietz JA, Morris TR. JHEP (2014) 03:093.

92. Dietz JA, Morris TR. JHEP (2015) 04:118.

93. Eichhorn A, Labus P, Pawlowski JM, Reichert M. SciPost Phys (2018a) 5:31. doi:10.21468/scipostphys.5.4.031

94. Baloitcha E, Lahoche V, Ousmane Samary D, (2020)

95. Nielsen NK, On the gauge dependence of spontaneous symmetry breaking in gauge theories. Nucl Phys B (1975) 101:173. doi:10.1016/0550-3213(75) 90301-6

96. Fukuda R, Kugo T. Gauge invariance in the effective action and potential. Phys Rev D (1976) 13:3469. doi:10.1103/physrevd.13.3469

97. Bürger B, Pawlowski JM, Reichert M, Schaefer B-J, (2019)

98. Reuter M, Wetterich C. Gluon condensation in nonperturbative flow equations. Phys Rev D (1997) 56:7893. doi:10.1103/physrevd.56.7893

99. Freire F, Litim DF, Pawlowski JM, Gauge invariance and background field formalism in the exact renormalisation group. Phys Lett B (2000) 495:256. doi:10.1016/s0370-2693(00)01231-4

100. Manrique E, Reuter M, Bimetric truncations for quantum Einstein gravity and asymptotic safety. Ann Phys (2010) 325:785. doi:10.1016/j.aop.2009.11.009

101. Manrique E, Reuter M, Saueressig F. Matter induced bimetric actions for gravity. Ann Phys (2011) 326:440. doi:10.1016/j.aop.2010.11.003

102. Becker D, Reuter M. En route to Background Independence: broken splitsymmetry, and how to restore it with bi-metric average actions. Ann Phys (2014) 350:225. doi:10.1016/j.aop.2014.07.023

103. Safari M. Eur Phys J (2016) C76:201.

104. Safari M, Vacca GP. Phys Rev (2017) D96:085001.

105. Safari M, Vacca GP. JHEP (2016) 11:139.

106. Lippoldt S. Renormalized functional renormalization group. Phys Lett B (2018) 782:275. doi:10.1016/j.physletb.2018.05.037

107. Christiansen N, Falls K, Pawlowski JM, Reichert M. Phys Rev (2018) D97: 046007.

108. Falls K, Litim DF, Nikolakopoulos K, Rahmede C. Phys Rev (2016) D93: 104022

109. Falls K, King CR, Litim DF, Nikolakopoulos K, Rahmede C. Phys Rev (2018) D97:086006.

110. Falls KG, Litim DF, Schröder. J. Phys Rev D (2019) 99:126015. doi:10.1103/ physrevd.99.126015

111. Demmel M, Saueressig F, Zanusso O. JHEP (2015) 08:113.

112. Taylor JC, Ward identities and charge renormalization of the Yang-Mills field. Nucl Phys B (1971) 33:436. doi:10.1016/0550-3213(71)90297-5

113. Slavnov AA, Ward identities in gauge theories. Theor Math Phys (1972) 10:99. doi:10.1007/bf01090719

114. Bonini M, D’Attanasio M, Marchesini G. Nucl Phys (1994) B421:429.

115. Bonini M, D’Attanasio M, Marchesini G. Nucl Phys (1995) B437:163.

116. Bonini M, D'Attanasio M, Marchesini G. Phys Lett (1995) B346:87.

117. Bonini M, D'Attanasio M, Marchesini G. Perturbative infrared finiteness of Yang-Mills theory from renormalization group flow. Nucl Phys B (1995) 444: 602. doi:10.1016/0550-3213(95)00166-p

118. Ellwanger U. Phys Lett (1994) B335:364.

119. D’Attanasio M, Morris TR. Phys Lett (1996) B378:213.

120. Litim DF, Pawlowski JM. On general axial gauges for QCD. Nucl Phys B Proc Suppl (1999) 74:329. doi:10.1016/s0920-5632(99)00188-7

121. Igarashi Y, Itoh K, So H. Phys Lett (2000) B479:336.

122. Igarashi Y, Itoh K, So H. Prog Theor Phys (2000) 104:1053.

123. Igarashi Y, Itoh K, So H. Prog Theor Phys (2001) 106:149.

124. Igarashi $\mathrm{Y}$, Itoh $\mathrm{K}$, So $\mathrm{H}$, Regularized quantum master equation in the Wilsonian. J High Energy Phys (2001b) 2001:032. doi:10.1088/1126-6708/ 2001/10/032

125. Igarashi Y, Itoh K, So H. Phys Lett (2002) B526:164

126. Igarashi $\mathrm{Y}$, Itoh $\mathrm{K}$, Sonoda $\mathrm{H}$. Quantum master equation for $\mathrm{QED}$ in exact renormalization group. Prog Theor Phys (2007) 118:121. doi:10.1143/ptp.118.121

127. Igarashi $\mathrm{Y}$, Itoh $\mathrm{K}$, Sonoda $\mathrm{H}$. Ward-takahashi identity for yang-mills theory in the exact renormalization group. Prog Theor Phys (2008) 120:1017. doi:10. $1143 /$ ptp. 120.1017

128. Igarashi Y, Itoh K, Sonoda H. Prog Theor Phys Suppl (2010) 181:1.

129. Sonoda H. J Phys (2013) A47:015401.
130. Igarashi $Y$, Itoh $K$, Pawlowski JM. Functional flows in QED and the modified Ward-Takahashi identity. J Phys Math Theor (2016) 49:405401. doi:10.1088/ $1751-8113 / 49 / 40 / 405401$

131. Asnafi S, Gies H, Zambelli L. Phys Rev (2019) D99:085009.

132. Igarashi Y, Itoh K, Morris TR. PT (2019) 2019:103B01. doi:10.1093/ptep/ ptz099

133. Pagani C. Phys Rev D (2016) 94:045001. doi:10.1103/physrevd.94.045001

134. Herbst TK, Luecker J, Pawlowski JM, (2015)

135. Pagani C, Reuter M. Phys Rev (2017) D95:066002.

136. Becker M, Pagani C, Zanusso O. Phys Rev Lett (2020) 124:151302. doi:10. 1103/physrevlett.124.151302

137. Houthoff W, Kurov A, Saueressig F. JHEP (2020) 04:099.

138. Kurov A, Saueressig F. Front Physiol (2020) 8:187. doi:10.3389/fphy.2020.00187

139. Litim DF, Pawlowski JM. Phys Lett (1998) B435:181.

140. Ambjorn J, Carfora M, Marzuoli A. The geometry of dynamical triangulations, Vol. 50 (1997).

141. Ambjorn J, Goerlich A, Jurkiewicz J, Loll R. Phys Rep (2012) 519:127.

142. Loll R. Classical Quant Grav (2020) 37:013002. doi:10.1088/1361-6382/ ab57c7

143. Hamber HW, Quantum gravity on the lattice. Gen Relat Gravit (2009) 41:817. doi:10.1007/s10714-009-0769-y

144. Eichhorn A, Koslowski T, Pereira A. Status of background-independent coarse graining in tensor models for quantum gravity. Universe (2019) 5 : 53. doi:10.3390/universe 5020053

145. Morris TR. Nucl Phys (2000) B573:97.

146. Morris TR. JHEP (2000) 12:012.

147. Arnone S, Kubyshin YA, Morris TR, Tighe JF, Gauge-invariant regularisation via su(n|n). Int J Mod Phys (2002) 17:2283. doi:10.1142/s0217751x02009722

148. Arnone S, Gatti A, Morris TR. Phys Rev (2003) D67:085003.

149. Arnone S, Morris TR, Rosten OJ. A generalised manifestly gauge invariant exact renormalisation group for SU(N) Yang-Mills. Eur Phys J C (2007) 50: 467. doi:10.1140/epjc/s10052-007-0258-y

150. Arnone S, Morris TR, Rosten OJ. Manifestly gauge invariant QED. J High Energy Phys (2005) 2005:115. doi:10.1088/1126-6708/2005/10/115

151. Morris TR, Rosten OJ. Phys Rev (2006) D73:065003.

152. Morris TR, Rosten OJ. J Phys (2006) A39:11657.

153. Rosten OJ. Phys Rev (2006) D74:125006.

154. Rosten OJ. Int J Mod Phys (2006) A21:4627.

155. Rosten OJ. Phys Lett (2007) B645:466.

156. Rosten OJ. Phys Lett (2008) B662:237.

157. Morris TR, Preston AWH. JHEP (2016) 06:012.

158. de Alwis SP. JHEP (2018) 03:118.

159. Bonanno A, Lippoldt S, Percacci R, Vacca GP. Eur Phys J C (2020b) 80:249. doi:10.1140/epjc/s10052-020-7798-9

160. Wetterich C. Gauge symmetry from decoupling. Nucl Phys B (2017) 915:135. doi:10.1016/j.nuclphysb.2016.12.008

161. Wetterich C. Gauge-invariant fields and flow equations for Yang-Mills theories. Nucl Phys B (2018) 934:265. doi:10.1016/j.nuclphysb.2018.07.002

162. Pawlowski JM, Reichert M, Wetterich C, Yamada M. Phys Rev D (2019) 99: 086010. doi:10.1103/physrevd. 99.086010

163. Wetterich C, (2019)

164. Wetterich C, Yamada M. Phys Rev D (2019) 100:066017. doi:10.1103/ physrevd.100.066017

165. Litim DF, Pawlowski JM. Phys Rev (2002) D66:025030.

166. Litim DF, Pawlowski JM. Phys Lett (2001) B516:197.

167. Mitter M, Pawlowski JM, Strodthoff N. Phys Rev (2015) D91:054035.

168. Cyrol AK, Fister L, Mitter M, Pawlowski JM, Strodthoff N. Phys Rev (2016) D94:054005.

169. Cyrol AK, Mitter M, Strodthoff N. FormTracer. A mathematica tracing package using FORM. Comput Phys Commun (2017) 219:346. doi:10.1016/j. cpc.2017.05.024

170. Cyrol AK, Mitter M, Pawlowski JM, Strodthoff N. Phys Rev (2018a) D97: 054006.

171. Corell L, Cyrol AK, Mitter M, Pawlowski JM, Strodthoff N. SciPost Phys (2018) 5:066. doi:10.21468/scipostphys.5.6.066

172. Falls K, Litim D, Nikolakopoulos K, Rahmede C, (2013) 
173. Falls K, Litim DF, Nikolakopoulos K, Rahmede C. On de Sitter solutions in asymptotically safe $\{\mathrm{f}(\mathrm{R})\}$ theories. Classical Quant Grav (2018) 35:135006. doi:10.1088/1361-6382/aac440

174. Codello A, Percacci R, Rahmede C. Ultraviolet properties of $\mathrm{f}(\mathrm{R})$-gravity. Int J Mod Phys (2008) 23:143. doi:10.1142/s0217751x08038135

175. Machado PF, Saueressig F. Phys Rev (2008) D77:124045.

176. Codello A, Percacci R, Rahmede C. Investigating the ultraviolet properties of gravity with a Wilsonian renormalization group equation. Ann Phys (2009) 324:414. doi:10.1016/j.aop.2008.08.008

177. Benedetti D, Caravelli F. JHEP (2012) 06:017.

178. Dietz JA, Morris TR. JHEP (2013a) 01:108.

179. Benedetti D. On the number of relevant operators in asymptotically safe gravity. EPL (2013) 102:20007. doi:10.1209/0295-5075/102/20007

180. Dietz JA, Morris TR. JHEP (2013b) 07:064.

181. Demmel M, Saueressig F, Zanusso O. JHEP (2014) 06:026.

182. Gonzalez-Martin S, Morris TR, Slade ZH. Phys Rev (2017) D95:106010.

183. Alkofer N. Asymptotically safe $\mathrm{f}(\mathrm{R})$-gravity coupled to matter II: global solutions. Phys Lett B (2019) 789:480 doi:10.1016/j.physletb.2018.12.061

184. Denz T, Pawlowski JM, Reichert M. Eur Phys J (2018) C78:336.

185. Fischer CS, Maas A, Pawlowski JM. On the infrared behavior of Landau gauge Yang-Mills theory. Ann Phys (2009) 324:2408. doi:10.1016/j.aop.2009.07.009

186. Hamada Y, Yamada M. JHEP (2017) 08:070.

187. Eichhorn A, Held A. Phys Rev (2017) D96:086025.

188. De Brito GP, Hamada Y, Pereira AD, Yamada M. JHEP (2019b) 08:142.

189. Falls K, Ohta N, Percacci R. Towards the determination of the dimension of the critical surface in asymptotically safe gravity. Phys Lett B (2020) 810: 135773. doi:10.1016/j.physletb.2020.135773

190. Codello A. Polyakov effective action from functional renormalization group equation. Ann Phys (2010) 325:1727. doi:10.1016/j.aop.2010.04.013

191. Corianò C, Maglio MM, Mottola E. TTT in CFT: trace identities and the conformal anomaly effective action. Nucl Phys B (2019) 942:303. doi:10.1016/ j.nuclphysb.2019.03.019

192. Pawlowski J. Exact flow equations and the U(1) problem. Phys Rev D (1998) 58:045011. doi:10.1103/PhysRevD.58.045011

193. Reuter M. Renormalization of the topological charge in yang-mills theory. Mod Phys Lett A (1997) 12:2777. doi:10.1142/s0217732397002922

194. Huber MQ, Braun J. Algorithmic derivation of functional renormalization group equations and Dyson-Schwinger equations. Comput Phys Commun (2012) 183:1290. doi:10.1016/j.cpc.2012.01.014

195. Huber MQ, Cyrol AK, Pawlowski JM. DoFun 3.0: functional equations in mathematica. Comput Phys Commun (2020) 248:107058. doi:10.1016/j.cpc. 2019.107058

196. Maas A. The Fröhlich-Morchio-Strocchi mechanism and quantum gravity. SciPost Phys (2020) 8:051. doi:10.21468/scipostphys.8.5.071

197. Christiansen N, Knorr B, Meibohm J, Pawlowski JM, Reichert M. Local quantum gravity. Phys Rev D (2015) 92:121501. doi:10.1103/PhysRevD.92. 121501

198. Christiansen N, Knorr B, Pawlowski JM, Rodigast A. Global flows in quantum gravity. Phys Rev D (2016) 93:044036. doi:10.1103/PhysRevD.93.044036

199. Christiansen N, Litim DF, Pawlowski JM, Rodigast A. Fixed points and infrared completion of quantum gravity. Phys Lett B (2014) 728:114. doi:10. 1016/j.physletb.2013.11.025

200. Christiansen N. Four-derivative quantum gravity beyond perturbation theory. Preprint arXiv:1612.06223 [hep-th] (2016)

201. Meibohm J, Pawlowski JM, Reichert M. Asymptotic safety of gravity-matter systems. Phys Rev D (2016) 93:084035. doi:10.1103/PhysRevD.93.084035

202. Christiansen N, Litim DF, Pawlowski JM, Reichert M. Asymptotic safety of gravity with matter. Phys Rev D (2018) 97:106012. doi:10.1103/PhysRevD.97.106012

203. Eichhorn A, Lippoldt S, Pawlowski JM, Reichert M, Schiffer M. How perturbative is quantum gravity? Phys Lett B (2019) 792:310. doi:10.1016/ j.physletb.2019.01.071

204. Eichhorn A, Lippoldt S, Schiffer M. Zooming in on fermions and quantum gravity. Phys Rev D (2019) 99:086002. doi:10.1103/physrevd.99.086002

205. Knorr B. Infinite order quantum-gravitational correlations. Classical Quant Grav (2018) 35:115005. doi:10.1088/1361-6382/aabaa0

206. Knorr B, Lippoldt S. Correlation functions on a curved background. Phys Rev (2017) D96:065020. doi:10.1103/PhysRevD.96.065020
207. Shaposhnikov M, Wetterich C. Asymptotic safety of gravity and the Higgs boson mass. Phys Lett B (2010) 683:196. doi:10.1016/j.physletb.2009.12.022

208. Eichhorn A, Hamada Y, Lumma J, Yamada M. Viability of quantum-gravity induced ultraviolet completions for matter Phys Rev D (2018) 97:086004. doi:10.1103/physrevd.97.086004

209. Eichhorn A, Held A. Top mass from asymptotic safety. Phys Lett B (2018) 777:217. doi:10.1016/j.physletb.2017.12.040

210. Eichhorn A, Versteegen F. Upper bound on the Abelian gauge coupling from asymptotic safety. J High Energ Phys (2018) 2018:030. doi:10.1007/ JHEP01(2018)030

211. Eichhorn A, Held A, Wetterich C. Quantum-gravity predictions for the finestructure constant. Phys Lett B (2018) 782:198. doi:10.1016/j.physletb.2018. 05.016

212. Eichhorn A, Held A. Mass difference for charged quarks from asymptotically safe quantum gravity. Phys Rev Lett (2018b) 121:151302. doi:10.1103/ physrevlett.121.151302

213. Eichhorn A, Held A, Wetterich C. Predictive power of grand unification from quantum gravity. J High Energ Phys (2020) 2020:111. doi:10.1007/ JHEP08(2020)111

214. Kwapisz JH. Asymptotic safety, the Higgs boson mass, and beyond the standard model physics. Phys Rev D (2019) 100:115001. doi:10.1103/ physrevd.100.115001

215. Reichert M, Smirnov J. Dark matter meets quantum gravity. Phys Rev D (2020) 101:063015. doi:10.1103/physrevd.101.063015

216. Alkofer R, Eichhorn A, Held A, Nieto CM, Percacci R, Schröfl M. Quark masses and mixings in minimally parameterized UV completions of the standard model. Ann Phys (2020) 421:168282. doi:10.1016/j.aop.2020.168282

217. Hamada Y, Tsumura K, Yamada M. Scalegenesis and fermionic dark matters in the flatland scenario. Eur. Phys. J. C (2020) 80:368. doi:10.1140/epjc/ s10052-020-7929-3

218. Eichhorn A, Pauly M. Safety in darkness: Higgs portal to simple Yukawa systems Preprint arXiv:2005.03661 [hep-ph] (2020).

219. Donà $\mathrm{P}$, Eichhorn A, Percacci R. Matter matters in asymptotically safe quantum gravity. Phys Rev D (2014) 89:084035. doi:10.1103/PhysRevD.89. 084035

220. Meibohm J, Pawlowski JM. Chiral fermions in asymptotically safe quantum gravity. Eur Phys J C (2016) 76:285. doi:10.1140/epjc/ s10052-016-4132-7

221. Henz T, Pawlowski JM, Wetterich C. Scaling solutions for Dilaton quantum gravity. Phys Lett B (2017) 769:105. doi:10.1016/j.physletb.2017.01.057

222. Eichhorn A, Held A, Pawlowski JM. Quantum-gravity effects on a HiggsYukawa model. Phys Rev D (2016) 94:104027. doi:10.1103/PhysRevD.94. 104027

223. Christiansen N, Eichhorn A. An asymptotically safe solution to the $U(1)$ triviality problem. Phys Lett B (2017) 770:154. doi:10.1016/j.physletb.2017. 04.047

224. Eichhorn A, Lippoldt S, Skrinjar V. Nonminimal hints for asymptotic safety. Phys Rev D (2018) 97:026002. doi:10.1103/PhysRevD.97.026002

225. Eichhorn A, Gies H, Scherer MM. Asymptotically free scalar curvature-ghost coupling in quantum einstein gravity. Phys Rev D (2009) 80:104003. doi:10. 1103/PhysRevD.80.104003

226. Eichhorn A, Gies H. Ghost anomalous dimension in asymptotically safe quantum gravity. Phys Rev D (2010) 81:104010. doi:10.1103/PhysRevD.81. 104010

227. Groh K, Saueressig F Ghost wavefunction renormalization in asymptotically safe quantum gravity. J Phys Math Theor (2010) 43:365403. doi:10.1088/ 1751-8113/43/36/365403

228. Eichhorn A, Gies H. Light fermions in quantum gravity. New J Phys (2011) 13:125012. doi:10.1088/1367-2630/13/12/125012

229. Eichhorn A. Quantum-gravity-induced matter self-interactions in the asymptotic-safety scenario. Phys Rev D (2012) 86:105021. doi:10.1103/ PhysRevD.86.105021

230. Codello A, D’Odorico G, Pagani C. Consistent closure of renormalization group flow equations in quantum gravity. Phys Rev D (2014) 89:081701. doi:10.1103/PhysRevD.89.081701

231. Eichhorn A. Faddeev-Popov ghosts in quantum gravity beyond perturbation theory. Phys Rev D (2013) 87:124016. doi:10.1103/PhysRevD.87.124016 
232. Eichhorn A, Lippoldt S. Quantum gravity and standard-model-like fermions. Phys Lett B (2017) 767:142. doi:10.1016/j.physletb.2017.01.064

233. Manrique E, Reuter M, Saueressig F. Bimetric renormalization group flows in quantum Einstein gravity. Ann Phys (2011) 326:463. doi:10.1016/j.aop.2010. 11.006

234. Tanabashi MParticle Data Group). Review of particle physics. Phys Rev D (2018) 98:030001. doi:10.1103/PhysRevD.98.030001

235. BonannoDenz T, Pawlowski JM, Reichert M (2020)

236. Becker D, Reuter M. Resonances and unitarity in weak Boson scattering at the LHC. J High Energ Phys (2014) 12:025. doi:10.1088/1126-6708/2008/11/010

237. Cyrol AK, Pawlowski JM, Rothkopf A, Wink N. Reconstructing the gluon. SciPost Phys (2018) 5:065. doi:10.21468/scipostphys.5.6.065

238. Knorr B, Saueressig F. Towards reconstructing the quantum effective action of gravity. Phys Rev Lett (2018) 121:161304. doi:10.1103/physrevlett.121. 161304

239. Bosma L, Knorr B, Saueressig F. Resolving spacetime singularities within asymptotic safety. Phys Rev Lett (2019) 123:101301. doi:10.1103/physrevlett. 123.101301

240. Knorr B, Ripken C, Saueressig F. Form factors in asymptotic safety: conceptual ideas and computational toolbox. Classical Quant Grav (2019) 36:234001. doi:10.1088/1361-6382/ab4a53

241. Draper T, Knorr B, Ripken C, Saueressig F (2020)

242. Draper T, Knorr B, Ripken C, Saueressig F (2020)

243. Dou D, Percacci R. The running gravitational couplings. Classical Quant Grav (1998) 15:3449. doi:10.1088/0264-9381/15/11/011

244. Percacci R, Perini D. Contraints on matter from asymptotic safety. Phys Rev D (2003) 67:081503. doi:10.1103/PhysRevD.67.081503

245. Granda LN. Nonperturbative renormalization group for Einstein gravity with matter. Europhys Lett (1998) 42:487. doi:10.1209/epl/i1998-00278-2

246. Granda L. Nuovo Cim B (1999) 114:509.

247. Donà $P$, Percacci R. Functional renormalization with fermions and tetrads. Phys Rev D (2013) 87:045002. doi:10.1103/PhysRevD.87.045002

248. Biemans J, Platania A, Saueressig F. Renormalization group fixed points of foliated gravity-matter systems. J High Energ Phys (2017) 2017:93. doi:10. 1007/JHEP05(2017)093

249. Gies H, Martini R. Curvature bound from gravitational catalysis. Phys Rev D (2018) 97:085017. doi:10.1103/physrevd.97.085017

250. Percacci R, Perini D. Asymptotic safety of gravity coupled to matter. Phys Rev D (2003) 68:044018. doi:10.1103/PhysRevD.68.044018

251. Narain G, Percacci R. Renormalization group flow in scalar-tensor theories. I. Classical Quant Grav (2010) 27:075001. doi:10.1088/0264-9381/27/7/075001

252. Zanusso O, Zambelli L, Vacca GP, Percacci R. Gravitational corrections to Yukawa systems. Phys Lett B (2010) 689:90. doi:10.1016/j.physletb.2010. 04.043

253. Vacca GP, Zanusso O. Asymptotic safety in Einstein gravity and scalarFermion matter. Phys Rev Lett (2010) 105:231601. doi:10.1103/physrevlett. 105.231601
254. Daum J-E, Harst U, Reuter M. Non-perturbative QEG corrections to the Yang-Mills beta function. Gen Relat Gravit (2010) 43:2393. doi:10.1007/ s10714-010-1032-2

255. Harst U, Reuter M. QED coupled to QEG. J High Energ Phys (2011) 2011:119. doi:10.1007/JHEP05(2011)119

256. Henz T, Pawlowski JM, Rodigast A, Wetterich C. Dilaton quantum gravity. Phys Lett B (2013) 727:298. doi:10.1016/j.physletb.2013.10.015

257. Oda K-y., Yamada M. Non-minimal coupling in Higgs-Yukawa model with asymptotically safe gravity. Classical Quant Grav (2016) 33:125011. doi:10. 1088/0264-9381/33/12/125011

258. Christiansen N, Eichhorn A, Held A. Is scale-invariance in gauge-Yukawa systems compatible with the graviton? Phys Rev D (2017) 96:084021.

259. Held A. Effective asymptotic safety and its predictive power: Gauge-Yukawa theories. arXiv:2003.13642 (hep-th) (2020)

260. Daas J, Oosters W, Saueressig F, Wang J. Asymptotically safe gravity with Fermions. Preprint arXiv: 2005.12356 (2020)

261. Gies H, Jaeckel J. Chiral phase structure of QCD with many flavors. Eur Phys J C (2006) 46:433. doi:10.1140/epjc/s2006-02475-0

262. Terao $\mathrm{H}$, Tsuchiya A. Conformal dynamics in gauge theories via non-perturbative renormalization group. Preprint arXiv:0704.3659v2 [hep-ph] (2007)

263. Braun J, Gies H. Scaling laws near the conformal window of many-flavor QCD. J High Energ Phys (2010) 2010:60. doi:10.1007/JHEP05(2010)060

264. Braun J, Fischer CS, Gies H. Beyond Miransky scaling. Phys Rev D (2011) 84: 034045. doi:10.1103/physrevd.84.034045

265. Codello A, Percacci R. Fixed points of higher-derivative gravity. Phys Rev Lett (2006) 97:221301. doi:10.1103/physrevlett.97.221301

266. Niedermaier MR. Gravitational fixed points from perturbation theory. Phys Rev Lett (2009) 103:101303. doi:10.1103/physrevlett.103.101303

267. Benedetti D, Machado PF, Saueressig F. Asymptotic safety in higherderivative gravity. Mod Phys Lett A (2009) 24:2233. doi:10.1142/ s0217732309031521

268. Ohta N, Percacci R. Higher derivative gravity and asymptotic safety in diverse dimensions. Classical Quant Grav (2014) 31:015024. doi:10.1088/0264-9381/ 31/1/015024

269. Ohta N, Percacci R, Pereira A. f(R, R2 $\mu v)$ at one loop. Phys Rev D (2018) 97: 104039. doi:10.1103/physrevd.97.104039

Conflict of Interest: The authors declare that the research was conducted in the absence of any commercial or financial relationships that could be constructed as a potential conflict of interest.

Copyright (c) 2021 Pawlowski and Reichert. This is an open-access article distributed under the terms of the Creative Commons Attribution License (CC BY). The use, distribution or reproduction in other forums is permitted, provided the original author(s) and the copyright owner(s) are credited and that the original publication in this journal is cited, in accordance with accepted academic practice. No use, distribution or reproduction is permitted which does not comply with these terms. 


\section{APPENDIX A NOTATION}

Our convention for functional derivatives are given by

$$
\frac{\delta J_{\mu_{1} \cdots \mu_{n}}(x)}{\delta J_{\nu_{1} \cdots \nu_{n}}(y)}=\frac{1}{\sqrt{\bar{g}}} \delta(x-y) \delta_{\mu_{1}}^{\left(\nu_{1}\right.} \cdots \delta_{\mu_{n}}^{\left.v_{n}\right)},
$$

where the parenthesis in the superscript of the Kronecker- $\delta$ 's stands for the symmetrisation of the indices including a normalisation factor $1 / n !$. For example we have

$$
\frac{\delta h_{\mu_{1} \mu_{2}}(x)}{\delta h_{\nu_{1} \nu_{2}}(y)}=\frac{1}{\sqrt{g}} \delta(x-y) \frac{1}{2}\left(\delta_{\mu_{1}}^{\nu_{1}} \delta_{\mu_{2}}^{\nu_{2}}+\delta_{\mu_{1}}^{\nu_{2}} \delta_{\mu_{2}}^{\nu_{1}}\right) .
$$

This leads to the correlation functions of the fluctuation fields as given in Ref. 14 .

The metric $\gamma^{a b}$ in field space is diagonal for bosons $\varphi$, and is symplectic for fermions $\psi, \bar{\psi}$,

$$
\left(\gamma_{\varphi}^{a b}\right)=1,\left(\gamma_{\psi}^{a b}\right)=\left(\begin{array}{cc}
0 & 1 \\
-1 & 0
\end{array}\right),
$$

with the Northwest-Southeast convention

$$
\phi^{a}=\gamma^{a b} \phi_{b}, \phi_{a}=\phi^{b} \gamma_{b a}
$$

These definitions entail

$$
\gamma_{b}^{a}=\delta_{b}^{a}, \text { and }\left(\gamma_{\varphi}\right)_{b}^{a}=\delta_{b}^{a},\left(\gamma_{\psi}\right)_{b}^{a}=-\delta_{b}^{a},
$$

more details can be found in Ref. 21 .

\section{APPENDIX B PONTRYAGIN INDEX IN U(1) GAUGE THEORIES}

The Pontryagin index $P$ of a four-dimensional $U(1)$-gauge theory in flat space is a simply example for a topological index in quantum field theory. For general field configurations it is a non-vanishing integer on manifolds such as $\mathbb{T}^{4}$, the four-dimensional torus, e.g., underlying standard lattice simulation. We write in general

$$
P[A, \theta]=\frac{1}{32 \pi^{2}} \int_{x} \theta(x) F_{\mu \nu} \tilde{F}^{\mu \nu}, \quad P[A, 1] \in \mathbb{Z},
$$

with the Pontryagin index $P[A]=P[A, \theta=1]$. The (dual) field strength, $F_{\mu \nu}$ and $\tilde{F}_{\mu \nu}$, are given by

$$
F_{\mu \nu}=\partial_{\mu} A_{\nu}-\partial_{\nu} A_{\mu}, \tilde{F}^{\mu \nu}=\frac{\epsilon^{\mu \nu \rho \sigma}}{2} F_{\rho \sigma} .
$$

In momentum space $P[A, \theta]$ reads

$$
P[A, \theta]=\frac{\epsilon^{\mu \nu \rho \sigma}}{16 \pi^{2}} \int_{p, q} \theta(-(p+q)) p_{\mu} A_{\nu}(p) q_{\sigma} A_{\rho}(q),
$$

The flow of $\theta$ has been studied in Ref. 193 for the topological charge in Yang-Mills theories. Two derivatives with respect to the gauge field in momentum space lead us from Eq. B8 to

$$
\frac{\delta P[A, \theta]}{\delta A_{\alpha}(p) \delta A_{\beta}(q)}=\frac{\epsilon^{\alpha \beta \rho \sigma}}{8 \pi^{2}} p_{\rho} q_{\sigma} \theta(-(p+q)) .
$$

For a topological term with constant $\theta=\theta_{\text {top }}$ we have $\theta(l)=\theta_{\text {top }}(2 \pi)^{4} \delta(l)$. Inserting this choice into Eq. B9, the term vanishes with $\epsilon^{\alpha \beta \rho \sigma} p_{\rho} p_{\sigma}=0$. 